\title{
Fuel Oil and Kerosene Sales, 1989
}

\author{
January 1991
}

\section{Energy Information Administration Office of Oil and Gas \\ U.S. Department of Energy \\ Washington DC 20585}

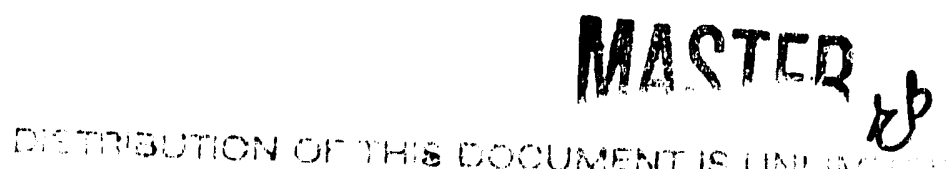

This report was prepared by the Energy Information Administration, the independent statistical and analytical agency within the Department of Energy. The information contained herein should not be construed as advocating or reflecting any policy position of the Department of Energy or any other viḡantization. 


\section{Contacts}

The Fuel Oil and Kerosene Sales. 1989 report is prepared in the Energy Information Administratton (I:LA) under the general direction of Michael Griffey (202-568-9601), Petroleum Marketing Division, (Office of ()il and Gas, F1A.

Detailed, technical questions about tables referencing the EIA-821 may be directed to:

Alice Lippert (202)586-9600 


\section{Contents}

Introduction .

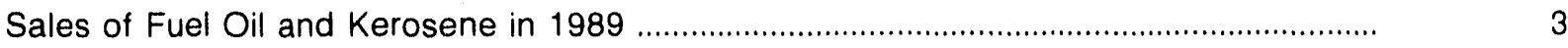

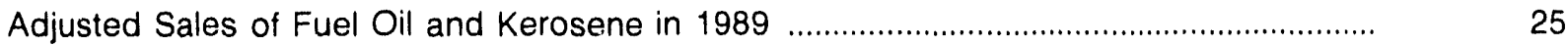

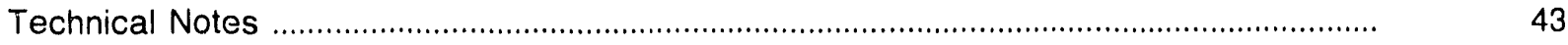

\section{Tables}

\section{Sales of Fuel Oil and Kerosene in 1989}

1. Sales of Distillate Fuel Oil by End Use in the United States: 1985-1989 .....................

2. Sales of Residual Fuel Oil by End Use in the United States: 1985-1989 .......................

3. Sales of Kerosene by End Use in the United States: 1985-1989 ............................... 10

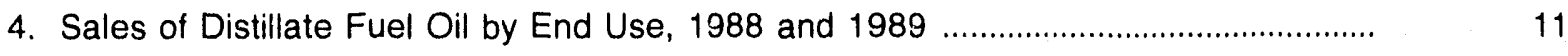

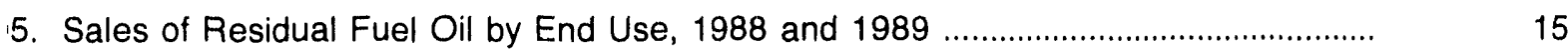

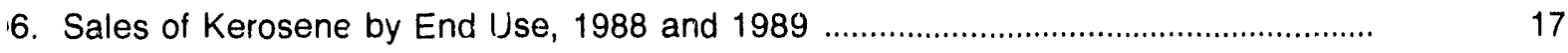

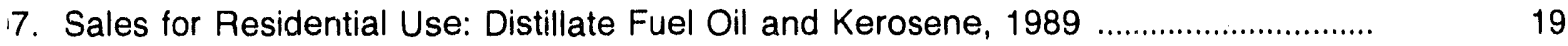

8. Sales for Commercial Use: Distillate Fuel Oil, Residual Fuel Oil, and Kerosene,

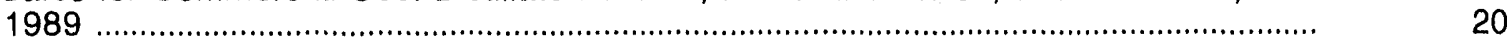

9. Sales for Industrial and Oil Company Use: Distillate Fuel Oil, Residual Fuel Oil, and
Kerosene, 1989

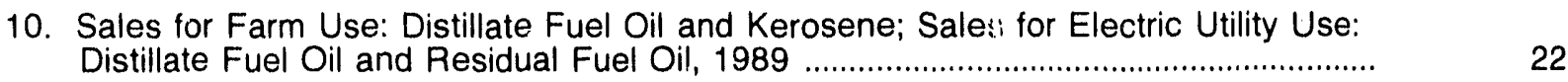

11. Sales for Transportation Use: Distillate Fuel Oil and Residual Fuel Oil, 1989 ............ 23

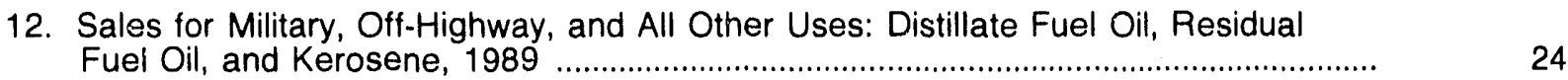




\section{Adjusted Sales of Fuel Oil and Kerosene in 1989}

13. Adjusted Sales of Distillate Fuel Oil by End Use in the United States: 1985-1989 .. 26

14. Adjusted Sales of Residual Fuel Oil by End Use in the United States: 1985-1989 .. 27

15. Adjusted Sales of Kerosene by End Use in the United States: 1985-1989 .............. 27

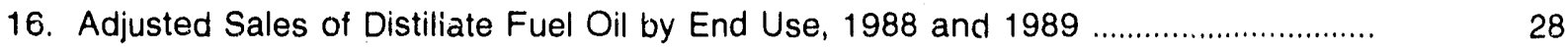

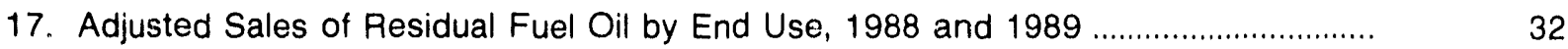

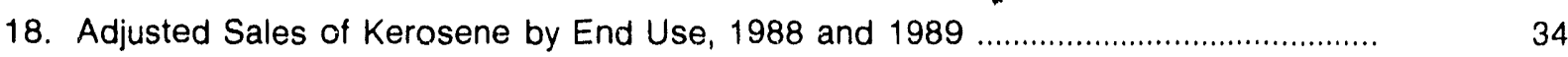

19. Adjusted Sales for Residential Use: Distillate Fuel Oil and Kerosene, 1989 .............. 36

20. Adjusted Sales for Commercial Use: Distillate Fuel Oil, Residual Fuel Oil and Kerosene, 1989

21. Adjusted Sales for Industrial and Oil Company Use: Distillate Fuel Oil, Residual Fuel Oil, and Kerosene, 1989

22. Adjusted Sales for Farm Use: Distillate Fuel Oil and Kerosene; Adjusted Sales for Electric Utility Use: Distillate Fuel Oil and Residual Fuel Oil, 1989 ..............................

23. Adjusted Sales for Transportation Use: Distillate Fuel Oil and Residual Fuel Oil, $1 ! 389$

24. Adjusted Sales for Military, Off-Highway, and All Other Uses: Distillate Fuel Oil, Residual Fuel Oil, and Kerosene, 1989

\section{Figures}

1. U.S. Sales of Distillate and Residual Fuel Oils by End Use, 1987-1989

2. Volume Distribution of Distillate and Residual Fuel Oils by PAD District, 1987-1989

3. Distillate and Residual Fuel Oil Sales for Largest End-Use Categories, by PAD District 


\section{Introduction}

This is the first presentation of statistics of fuel oil and kerosene sales as a stand-alone publication. Prior to this report the statistics appeared in the Petroleum Marketing Anmual (PMA) for reference year 1988 and the Petroleum Marketing Monthly (PMM) for reference years 1984 through 1987. This is the sixth annual presentation of the results of the ongoing "Annual Fuel Oil and Kerosene Sales Report," (Form ElA-821) survey.

Except for the kerosene and on-highway diesel information, data presented in Tables 1 through 12 (Sales of Fuel Oil and Kerosene) present results of the EIA-821 survey. Tables 13 through 24 (Adjusted Sales of Fuel Oil and Kerosene) include volumes which are based on the EIA-821 survey but have been adjusted at the Petroleum Administration for Defense (PAD) district level to equal the products supplied volumes published in the Petroleum Supply Annual (PSA). Please note: Tables 13 through 24 were formerly titled "Deliveries." However, due to confusion over the different titles in the two sets of tables, the titles in Tables 13 through 24 were changed to better reflect the intent of the data. This was a request made by the user community.

Tables 1 through 12 contain sales estimates from the EIA-821 survey based on data reported in the survey for all categories except kerosene and on-highway diesel. For on-highway diesel the survey was not expected to yield valid statistics because the frame does not include all truck stops. Hence, State-level data obtained from the Federal Highway Administration were used instead.

Kerosene sales data were not expected to be complete because a comprehensive listing of kerosene retailers was noi available to serve as a sampling frame. It was thought that a complete frame would be expensive to construct because many kerosene retailers are convenience stores or other small businesses. Because of these concerns, kerosene sales data have been published only after adjusting the sales data so that they add to the U.S. total of kerosene product supplied from the PSA. The 1989 U.S. total sales volume for kerosene from the survey was 931,016 thousand gallons. For Tables 1 through 12, this figure was adjusted to the PSA figure of 1,291,668 thousand gallons, an adjustment factor of 1.387 . The distillate and residual fuel oil sales totals were less than 4 percent greater than the products supplied totals, and were not adjusted at the national level.

Recent investigations in five States showed that in those States missing units contributed only a small volume of kerosene sales. The same investigation showed that another significant reason for the differences betweer, kerosene sales and kerosene product supplied is product fungibility. Kerosene, kerosene-type jet fuel, and No. 1 fuel oil can be used interchangeably, which explains a large part of the difference between kerosene product supplied and kerosene sales. In addition, in colder climates, kerosene is blended with distillate fuel and sold as fuel oil, resulting in less kerosene sold than supplied.

In Tables 13 through 24, PAD district-level estimates of distillate and residual fuel oils, and kerosene are adjusted to equal published PSA volume estimates of products supplied data. For certain sales categories, data obtained from alternate sources are used instead of the adjusted numbers. See "Technical Note 3" for further explanation. The sales data have been adjusted in this way and published by the Energy Information Administration (EIA) since 1978. Initially, the adjust ment was done because EIA did not believe that the data from the sales survey were reliable. However, significant improvements were made to the sampling frame and sample in 1984. EIA is continuing to publish the adjusted sales data because of historical precedent.

For the reasons mentioned above (i.e., frame undercoverage, product fungibility, and blending), the adjustment factors for kerosene by PAD district for 1989 are in some PAD districts much larger than the national adjustment factor as shown below. Adjustment factors for distillate and residual fuel oil are presented in "Technical Note 3."

\section{Kerosene Adjustment Factor}

- PAD District I: .68

- PAD District II: 1.50

- PAD District III: 14.16

- PAD District IV: 67

- PAD District V: 2.28 
The sales data (Tables 1 through 12) differ from the adjusted sales estimates (Tables 13 through 24) for many reasons, including:

- Some products are interchangeable (fungible) and may be supplied as one product and sold as another product. For example, as stated atove, kerosene, kerosene-type jet fisel, and No. 1 fuel oil can be ased interchangeably.

- Products supplied into a PAD district may be blended prior to final sale. For example, residual fuel and No. 2 distillate may be blended and sold as No. 4 fuel oil or, in colder climates, kerosene may be blended with distillate fuel oil and sold as heating oil.
- Geographic differences can be attributed to the transportation of product by truck or rail from the district of production. Infer-PAD-district movements of products by these modes of transportation are not accounted for in Tables 13 through 24.

- Products may be supplied into a PAD district but the final sale may cross PAD district boundaries. For example, a fuel oil dealer in Ohio (PAD District II) may make retail sales into Pennsylvania (PAD District IB) and/or West Virginia (PAD District IC)

- Drawdowns or buildups in stocks will cause volumes supplied to differ from sales volumes. 


\section{Sales of Fuel Oil and Kerosene in 1989}

\section{Overview}

Despite the rise in petroleum products prices, ' a colderthan-normal winter in the latter part of $1989^{2}$ spurred an increase in demand for distillate fuel oils. The shipping and electric utilities industries contributed to a significant rise in demand for both distillate and residual fuels oils in 1989.

A total of 72.9 billion gallons of fuel oil and kerosene were sold to consumers in 1989, an increase of 3.0 percent over 1988 sales volumes. Of all fuel oil sold during 1989. distillate fuel oil accounted for 68.3 percent, which was an increase over 1988 when distillate fuel oil accounted for 67.2 percent of all fuel oil products sold in the United States. Residual fuel oil's share of total fuel oil sold fell slightly to 29.9 percent from 30.7 percent in 1988. Kerosene followed with a 1.8 percent share, also falling from the previous year when it accounted for a 2.1 percent share of total fuel oil sold.

\section{Distillate Fuel Oil}

Distillate fuel oil sales reached another all-time high, with approximately 49.8 billion gallons sold in 1989 . This was a 4.6 percent increase over the previous year's sales. As in past years, transportation sales dominated total distillate sales, with a 53.4 percent share, an increase of 0.6 percentage points over 1988 (Figure 1). Of the transportation sales, on-highway diesel accounted for the largest volume of sales with 21.1 billion gallons sold; its share of total distillate sales also increased from 42.1 percent in 1988 to 42.4 percent in 1989. The majority of all distillate end-use sectors showed an increase in sales over 1988. The largest sales increases occurred in vessel bunkering and electric utility sectors, where customers increased their purchases by 13.4 and 23.3 percent, respectively, in 1989.

Residential sales rose 1.5 percent in 1989; however, their share of total distillate sales fell from 17.9 percent in 1988 to 17.4 percent in 1989. Sales to industrial and farm customers rebounded from 1988 by showing an increase of 6.4 and 6.2 percent, respectively. Industrial and farm distillate shares also increased during this time period from 5.4 to 5.5 percent and 6.6 to 6.7 percent, respectively.

Figure 2 depicts the volume distribution of distillate fuel oil by Petroleum Administration Defense (PAD) districts for 1987-1989. PAD District I, the East Coast, accounted for the majority of sales for this time period. However, its share of these sales declined for the second straight year, down from 42.5 percent in 1987 to 40.7 percent in 1989. PAD District II, the Midwest, showed an increase in its share of distillate sales from 28.1 in 1987 to 28.8 percent in 1989. Distillate fuel oil sales increased the most in PAD District III, the Gulf Coast, up 8.4 percent. The percentage share of total distillate sales remained basically the same for PAD District IV, the Rocky Mountain Region, and PAD District V, the West Coast, during 1988 and 1989.

\section{Residual Fuel Oil}

Although prices of residual fuel oil rose in 1989 , sales of residual fuel oil increased for the second straight year. Electric utilities and shippers continued their demand for large quantities of residual fuel oil in 1989, particularly ship owners, whose purchases increased 13.2 percent over 1988. The share of vessel bunkering sales relative to total residual fuel oil sales also increased from 29.3 percent in 1988 to 32.9 percent in 1989.

Sales of residual fuel oil to electric utilities increased slightly in 1989 (2.3 percent). Competitive prices in early 1989 gave electric utility companies the option of using residual fuel oil to mcet customer demand. As prices of residual fuel oil continued to climb during the year, their purchases of residual fuel oil tapered off; however, their share of total residual fuel oil sales increased in 1989, up 0.7 percentage points from 1988.

The majority of residual fuel oil sales to electric utilities remained on the East Coast, PAD District I, with an 87.8 percent share of all electric utility residual fuel oil sales (Figure 3). This was an increase over 1988 when the share was 84.4 percent; however, this was still below the share of 1987 when PAD District I accounted

\footnotetext{
'Energy Information Administration, Petroleum Marketing Monthly, DOE/EIA-0380(89/12) (Washington, D.C., February 1990), Tables 2 and $4, \mathrm{pp} .14$ and 16.

2Energy Information Administration, Short-Term Energy Outlook. DOE/EIA-020(90/2Q) (Washington, D.C., February 1990), Table 4, p. 42.
} 
for a 90.8 percent share of electric utility residual fuel oil sales

Higher residual fuel oil prices also had an effect on other end-use sales of residual fuel oil. In particular, oil company, industrial" and commercial end-use sales which fell $31.8,17.7$, and 7.0 percent, respectively. from 1988. Because natural gas prices fell during midyear 1989, industrial and commercial end-users opted to switch to natural gas for space heating and operating equipment.

The majority of residual fuel oil sales were made in PAD District I, the East Coast (Figure 2). The share of total residual fuel oil sold in this PAD district increased from 60.2 percent in 1988 to 61.4 percent in 1989. PAD District III, the Gulf Coast, showed an increase in its share of residual fuel oil brought on mostly by an increase of vessel bunkering sales in this area. Vessel bunkering sales in this region were up
17.0 pereent over 1988. PAD District V, the West Coast, also showed an increase in vessel bunkering sales; however, the overall share of residual fuel oil sales in this PAD district fell 1.6 percentage points from 1988 .

\section{Kerosene}

Kerosene sales fell in 1989 after making a come back in 1988. Overall sales of kerosene decreased by 12.6 percent in 1989. The majority of sales were made to residential and commercial customers, 67.3 and 15.8 percent, respectively. While the share of kerosene sales to residential end users fell 4.6 percentage points from 1988 , the share of sales to commercial end users increased 2.8 percentage points during this same time period. As in past years, PAD Districts I and II (the East Coast and Midwest) accounted for the bulk of kerosene sales.

${ }^{3}$ Industrial residual fuel oil sales decreased by 17.7 percent in 1989 . During the validation of these data, it was found that some companies had misclassified their industrial residual fuel oil sales in 1988. In 1989, these sales were reported as vessel bunkering residual fuel oil sales. Approximately 200 million gallons of residual fuel oil sales were incorrectly reported as industrial end-use sales in 1988. Hence, residual fuel oil sales would not have shown this large decrease in 1989 had these volumes been correctly reported in 1988. 
Figure 1. U.S. Sales of Distillate and Residual Fuel Oils by End Use, 1987-1989
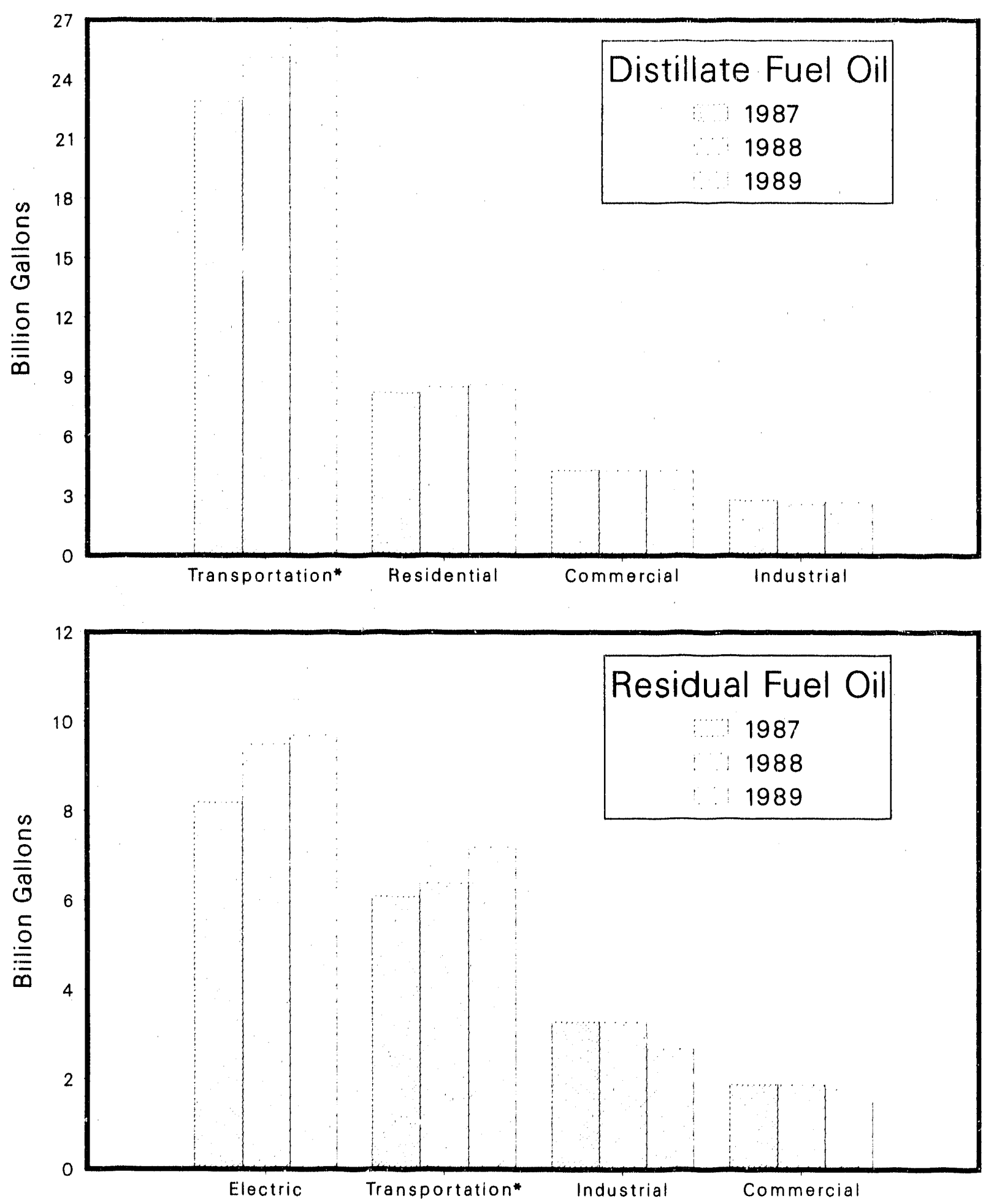

"For distillate fuel oil, transportation use comprises railroad, vessel bunkering. and on-highway diesel end-use categories. For residual fuel oil, transportation use comprises railroad and vessel bunkering end-use categories.

Sources: Energy Information Administration. "Fuel Oil and Kerosene Sales. $1989, "$ Tables 1 and 2. 
Figure 2. Volume Distribution of Distillate and Residual Fuel Oils b PAD District, 1987-1989
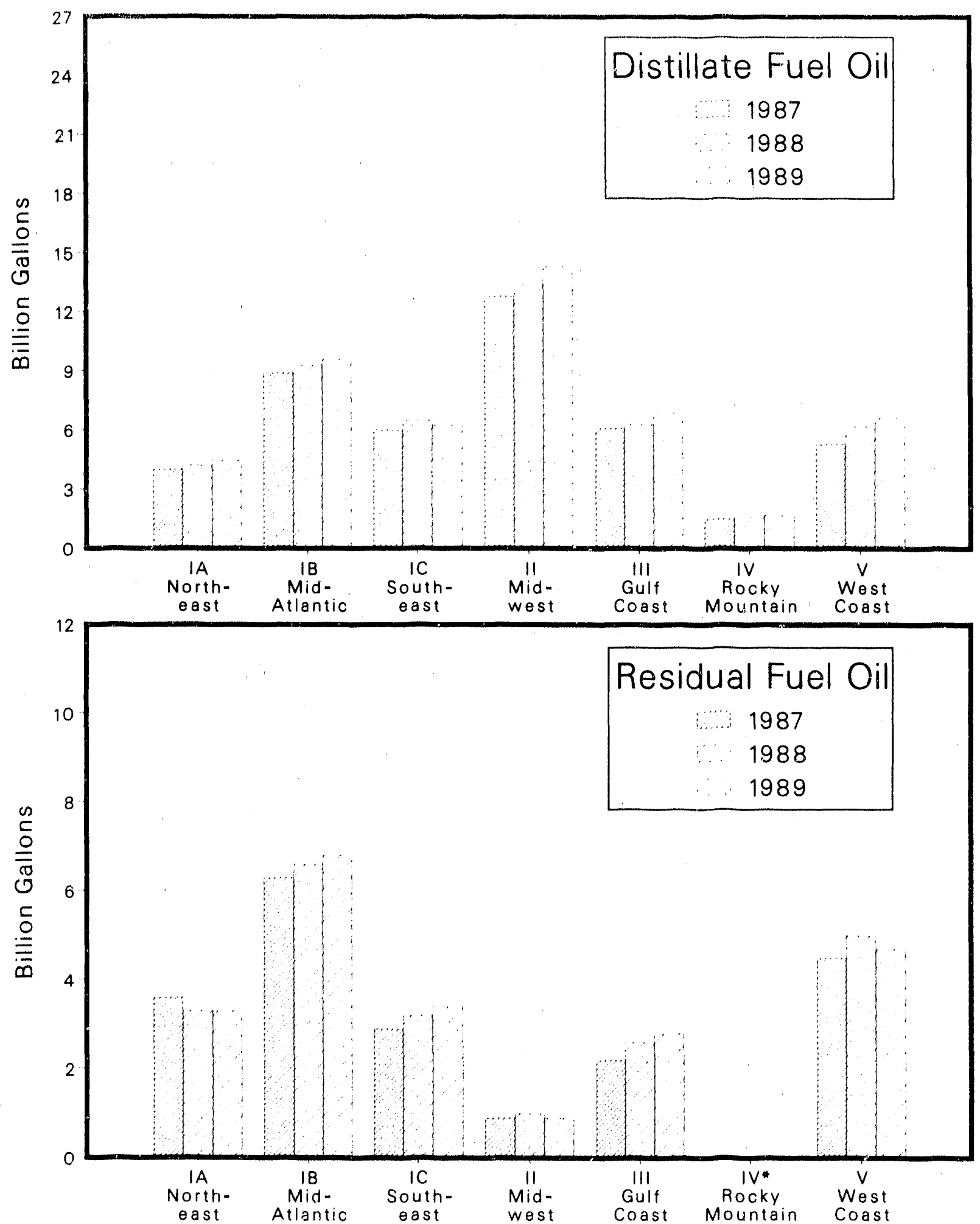

* Residual fuel oil sales in PAD District IV were too small to appear in this giaph. Sources: Energy Information Administration. "Fuel Oil and Kerosene Sales, 1989," Tables 4 and 5 . "Petroleum Marketing Monthly." June 1988, Tables A4 and Á5. 
Figure 3. Distillate and Residual Fuel Oil Sales for Largest End-Use Categories, by PAD District

Distillate: Transportation
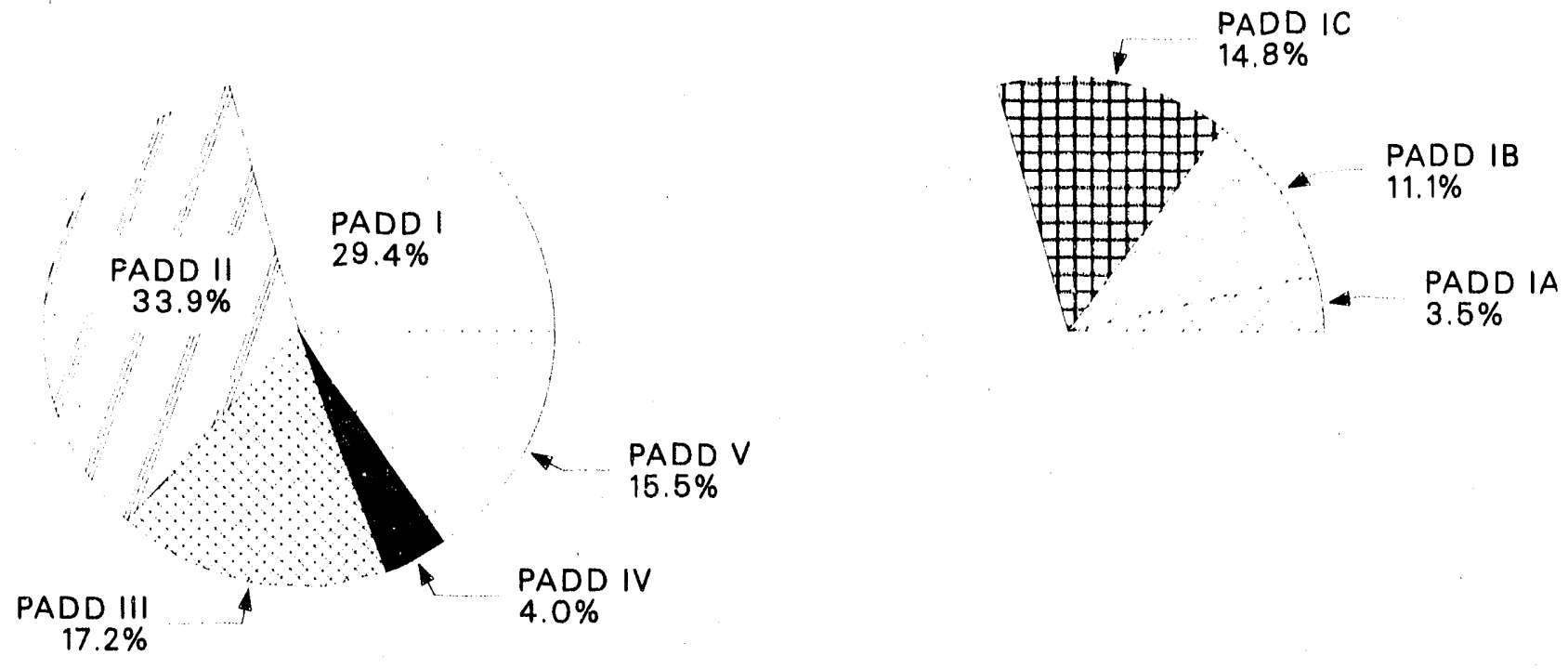

Residual: Electric Utility

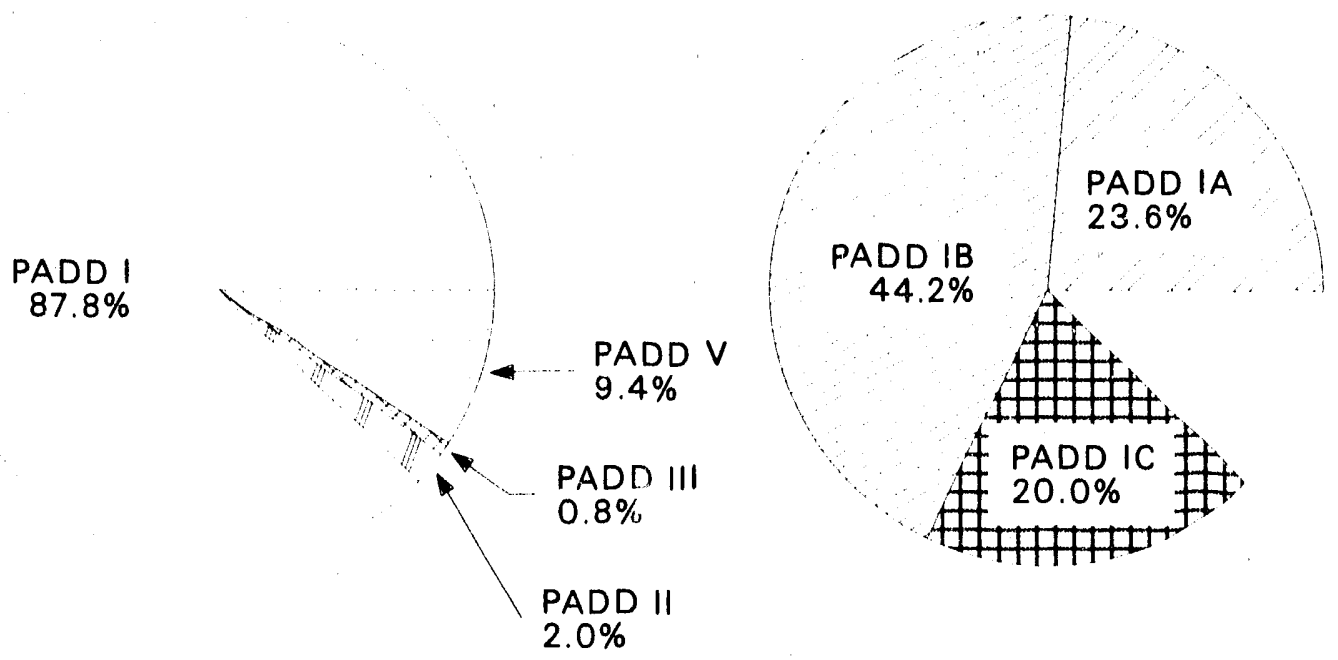

Source: Energy Information Administration. "Fuel Oil and Kerosene Sales, 1989," Tables 4 and 5. 
Table 1. Sales of Distillate Fuel Oil by End Use in the United States: 1985-1989 (Thousand Gallons)

\begin{tabular}{|c|c|c|c|c|c|}
\hline \multirow[b]{2}{*}{ End Use } & \multicolumn{5}{|c|}{ Distillate Fuel OII } \\
\hline & 1985 & 1986 & 1987 & 1988 & 1989 \\
\hline 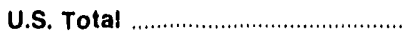 & $43,058,711$ & $45,124,491$ & $44,719,327$ & $47,576,811$ & $49,788,896$ \\
\hline 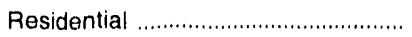 & $7,728,057$ & $8,098,496$ & $8,179,134$ & $8,518,859$ & $8,648,629$ \\
\hline 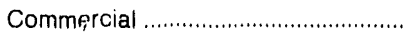 & $4,463,226$ & $4,542,253$ & $4,299,580$ & $4,294,182$ & $4,277,105$ \\
\hline 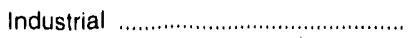 & $2,440,661$ & $2,681,720$ & $2,823,679$ & $2,562,685$ & $2,725,774$ \\
\hline 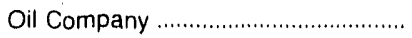 & 684,227 & 623,004 & 618,193 & 622,736 & 683,683 \\
\hline Farm & $3,102,106$ & $3,340,8 ! 3$ & $2,998,681$ & $3,162,575$ & $3,360,092$ \\
\hline 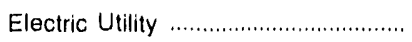 & 523,010 & 583,579 & 562,665 & 720,137 & 887,599 \\
\hline 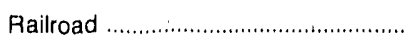 & $2,786,479$ & $2,850,311$ & $2,850,159$ & $3,095,736$ & $3,240,649$ \\
\hline 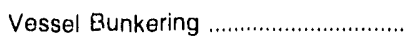 & $1,698,985$ & $1,849,423$ & $1,864,992$ & $1,991,773$ & $2,258,465$ \\
\hline 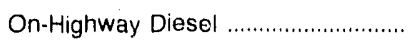 & $17,279,650$ & $17,926,508$ & $18,172,934$ & $20,038,691$ & $21,131,692$ \\
\hline 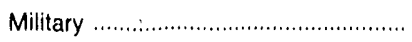 & 661,644 & 720,880 & 712,822 & 830,614 & 852,839 \\
\hline 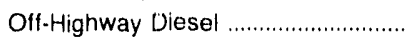 & $1,522,041$ & $1,659,365$ & $1,559,873$ & $1,671,387$ & $1,689,651$ \\
\hline 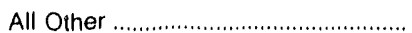 & 168,625 & 148,138 & 76,615 & 67,435 & 32,719 \\
\hline
\end{tabular}

Note: Totals may not equal sum of components due to independent rounding.

Sources: - Energy Ir. ormation Administration Form ElA-821. "Annual Fuel Oil and Kerosene Sales Report," for $1985-1989$.

- On-Highway Diesel data are Federal Highway Administation statistics of highway special fuels use. 
Table 2. Sales of Residual Fuel Oil by End Use in the United States: 1985-1989 (Thousund Gallons)

\begin{tabular}{|c|c|c|c|c|c|}
\hline \multirow{3}{*}{ End Use } & \multicolumn{5}{|c|}{ Residual Fuel Oil } \\
\hline & & & & & \\
\hline & 1985 & 1986 & 1987 & 1988 & 1989 \\
\hline . & $16,069,860$ & $22,407,894$ & $20,414,122$ & $21,719,226$ & $21,629,300$ \\
\hline 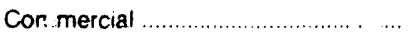 & $1,365,876$ & $1,996,238$ & $1,877,933$ & $1,927,533$ & $1,792,634$ \\
\hline 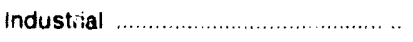 & $3,344,772$ & $4,0,35,217$ & 3.324 .337 & $3,239,538$ & $2,665,438$ \\
\hline (2) & $944, n 88$ & 803.875 & 669,176 & 548,930 & 374,459 \\
\hline 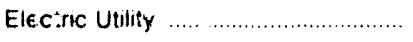 & 5.507 .617 & $9,3,5,361$ & $8,237,163$ & $9,464,457$ & $9,680,974$ \\
\hline 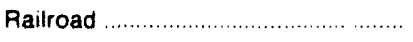 & 5,973 & 583 & 430 & 32 & 39 \\
\hline 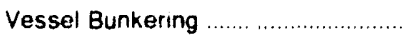 & $4,59 C, 013$ & $5,889,476$ & $6,1 \cup 5,089$ & $6,354,536$ & $7,191,689$ \\
\hline 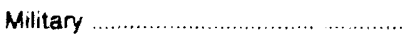 & 116,224 & 102,555 & 152,028 & 125,318 & 92,229 \\
\hline 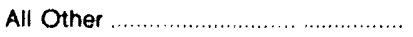 & 194,496 & 234,584 & 47,966 & 58,880 & 31,838 \\
\hline
\end{tabular}

Nole: Tolais may not equal sum of components due to independent rounding.

Sources: - Energy Information Adminisiration Form E:A.821. "Annual Fuel Oil and Kerosene Sales Report," for 1985.1989.

Tabie 3. Sales of Ker.jsene by End Use in the United States: 1985-1989 (Thousand Gal!' yii)!

\begin{tabular}{|c|c|c|c|c|c|}
\hline \multirow{3}{*}{ End Use } & \multicolumn{5}{|c|}{ Kerosent } \\
\hline & & & & & \\
\hline & 1985 & 1986 & 1987 & 1988 & 1889 \\
\hline LI.S. Total .. & $1,745,856$ & $1,506,162$ & $1,449,504$ & $1,478,316$ & $1,291,668$ \\
\hline 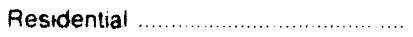 & $1,174,226$ & 898.625 & 879,406 & $1,063,187$ & 368,728 \\
\hline Commer cial ...................................... & 243,218 & 369,800 & 360,413 & 193.476 & 204,258 \\
\hline 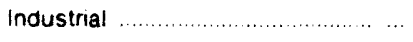 & 149,450 & 111,482 & 86.020 & 117,853 & 124,003 \\
\hline$(\ldots \ldots \ldots \ldots \ldots \ldots \ldots$ & 41,248 & 37,824 & $32,0.25$ & 33,426 & 30,081 \\
\hline All Other & 137,715 & 88,431 & 91,641 & 70,370 & 64,598 \\
\hline
\end{tabular}

Note Totals moy not equal sum of components due to independen rounding.

Note: Kerosene daia in the Sales tables $(1-12)$ are adjustod at the national level. See Technical Note $\mathcal{u}$ for further axplanation.

Sources. - Kerosene data are based on data from the Energy Information Ad-.nistration's "Petroleum Supply Annual," Volume 1, 1985.1989. (DOE/EIA-0340(B5)/1 - (89)/1) 
Table 4. Sales of Distillate Fuel Oil by End Use, 1988 and 1989 (Thousand Gallons)

\begin{tabular}{|c|c|c|c|c|c|c|}
\hline \multirow{3}{*}{ Destination } & \multicolumn{2}{|c|}{ Residential } & \multicolumn{2}{|c|}{ Commercial } & \multicolumn{2}{|c|}{ Industrial } \\
\hline & & & & & & \\
\hline & 1988 & 1989 & 1988 & 1989 & 1988 & 1989 \\
\hline 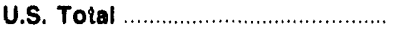 & $8,518,859$ & $8,648,629$ & $4,294,182$ & $4,277,105$ & $2,562,685$ & $2,725,774$ \\
\hline 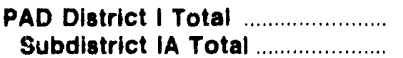 & $\begin{array}{l}6,842,982 \\
2,237,744\end{array}$ & $\begin{array}{l}6,901,689 \\
2,417,886\end{array}$ & $\begin{array}{r}2,694,430 \\
660,007\end{array}$ & $\begin{array}{r}2,854,869 \\
747,555\end{array}$ & $\begin{array}{r}1,003,201 \\
203,785\end{array}$ & $\begin{array}{l}973,890 \\
208,952\end{array}$ \\
\hline Connecticut & 627,071 & 686,835 & 144,332 & 165,454 & $\begin{array}{r}203,100 \\
47,225\end{array}$ & $\begin{array}{r}00,902 \\
44,923\end{array}$ \\
\hline 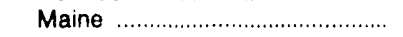 & 284,972 & 274,107 & 85,363 & 78,242 & 29,249 & 31.487 \\
\hline 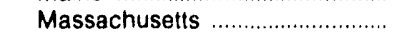 & 884,850 & 991,111 & 303,731 & 374,122 & 93,147 & 97,355 \\
\hline New Hampshire ............................ & 176,247 & 207,964 & 55,055 & 57,275 & 14,076 & 14,831 \\
\hline 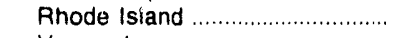 & 171,943 & 153,441 & 38,557 & 37,598 & 10,983 & 10,615 \\
\hline Vermont & 92,661 & 104,429 & 32,970 & 34,865 & 9,106 & 9,742 \\
\hline 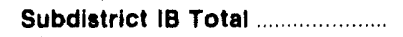 & $3,880,588$ & $3,831,255$ & $1,480,067$ & $1,630,541$ & 420,186 & 368,184 \\
\hline 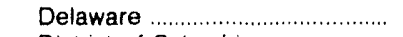 & 66,498 & 63,785 & 18,637 & 14,385 & 9,545 & 10,270 \\
\hline District of Columbia .................... & 14,089 & 7,029 & 26,124 & 26,060 & 47 & 122 \\
\hline 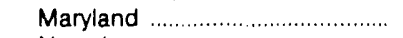 & 282,633 & 248,079 & 88,871 & 96,750 & 32,205 & 33,073 \\
\hline 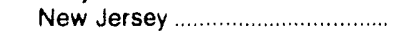 & 834,469 & 768,811 & 377,096 & 394,225 & 95,636 & 80,499 \\
\hline 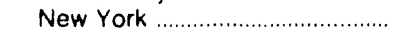 & $1,738,701$ & $1,679,326$ & 702.709 & 746,919 & 62,661 & 71,328 \\
\hline 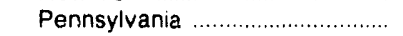 & 944,198 & $1,064,225$ & 266,630 & 352,203 & 220,092 & 172,892 \\
\hline Subdistrict IC Total & 724,651 & 652,549 & 554,355 & 476,773 & 379,229 & 396,754 \\
\hline Florida & 24,931 & 17.517 & 158,091 & 134,121 & 38,669 & 45,937 \\
\hline Georgia & 12,302 & 12,884 & 57,417 & 47,056 & 42,006 & 50,211 \\
\hline North Carolina & 273,796 & 225,742 & 147,362 & 113,509 & 92,097 & 93,239 \\
\hline South Carolina & 53,464 & 61,869 & 50,337 & 44,673 & 24,788 & 28,586 \\
\hline Virginia & 332,782 & 303,435 & 124,082 & 113,531 & 88,088 & 85,089 \\
\hline West Virginia & 27,375 & 31,102 & 17,066 & 23,883 & 93,581 & 93,691 \\
\hline 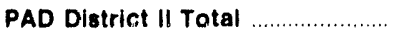 & $1,373,843$ & $1,404,069$ & $750,6 \$ 7$ & 693,799 & 751,713 & 794,541 \\
\hline 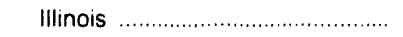 & 93,160 & 79.973 & $85.9(16$ & 71,308 & 75,758 & 91,162 \\
\hline 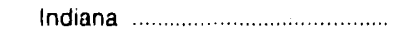 & 122,680 & 117,061 & 58,7411 & 58,438 & 74,847 & 102,287 \\
\hline 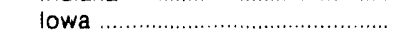 & 48,985 & 53,894 & 30,0811 & 24.778 & 20,685 & 16,214 \\
\hline 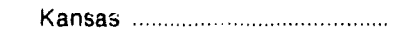 & 1,312 & 1,620 & 16,918 & 16.870 & 14,843 & 12,531 \\
\hline 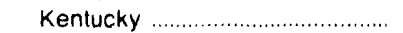 & 38,958 & 37.696 & 42,848 & 32,845 & 159,574 & 168,586 \\
\hline 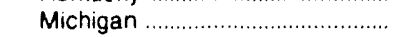 & 255,530 & 241,391 & 106,696 & 105,118 & 82,164 & 60,339 \\
\hline Minnesota & 192,125 & 227,397 & 48,378 & 52,285 & 43,523 & 44,337 \\
\hline 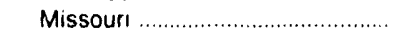 & 27,853 & 23,967 & 73,041 & 46,851 & 40,357 & 40,278 \\
\hline Nebraska & 8,736 & 12,598 & 13,128 & 11,519 & 5,350 & 6,080 \\
\hline 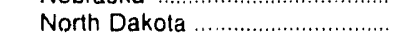 & 12,382 & 46,192 & 6,763 & 9,402 & 13,217 & 21,476 \\
\hline 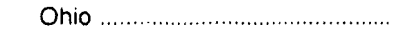 & 204,442 & 231,366 & 97,982 & 85,349 & 111,330 & 134,230 \\
\hline Oklahoma & 1,225 & 20 & 27,411 & 32,264 & 25,807 & 26,845 \\
\hline South Dakota & 40,390 & 45,547 & 15,157 & 11,148 & 8,794 & 6,198 \\
\hline Tennessee & 9,909 & 12.373 & 48,428 & 33,615 & 44,606 & 38,175 \\
\hline 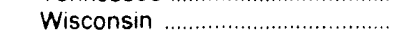 & 286,156 & 272,972 & 79,220 & 102,007 & 30,857 & $25,80 \%$ \\
\hline PAD District III Total. & 2,493 & 2,231 & 249,396 & 238,661 & 274,176 & 316,694 \\
\hline 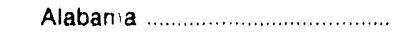 & 1,117 & 1.055 & 29,775 & 37,727 & 55,691 & 59,432 \\
\hline Arkans 35 & 35 & 23 & 6,734 & 13,515 & 12,796 & 15.765 \\
\hline 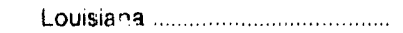 & 63 & 237 & 34,309 & $25,96 \%$ & 22,875 & 17,725 \\
\hline 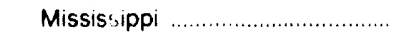 & 114 & 200 & 15,869 & 26,273 & 21,514 & 17,242 \\
\hline 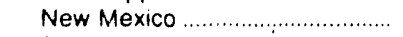 & 324 & 323 & 14,859 & 15,544 & 44,856 & 32,365 \\
\hline 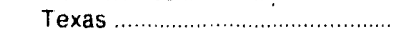 & 841 & 394 & 147,850 & 119,634 & 116.445 & 173,164 \\
\hline PAD District IV Total ........................ & 40,389 & 48,390 & 99,199 & 73,666 & 154,699 & 192,731 \\
\hline 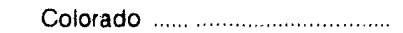 & 2,005 & 1,709 & 38,625 & 22,055 & 1,819 & 34,375 \\
\hline Idaho & 23,335 & 22,843 & 16,359 & 14,257 & .079 & 29,332 \\
\hline 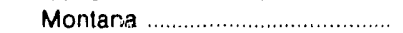 & 8,896 & 14,991 & 7,551 & 8,343 & 1,368 & 44,728 \\
\hline 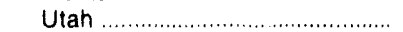 & 4,985 & 7,488 & 26,446 & 18,796 & 36,910 & 31,308 \\
\hline Wyoming & 1,168 & 1,361 & 10,218 & 10,216 & 42,523 & 52,988 \\
\hline 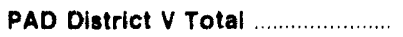 & 259,151 & 292,250 & 500,461 & 416,111 & 378,896 & 447,917 \\
\hline Alaska & 49,864 & 66,705 & 33,963 & 36,238 & 25,527 & 37,130 \\
\hline Arizona & 244 & 296 & 18,839 & 16,445 & 34,487 & 62,315 \\
\hline 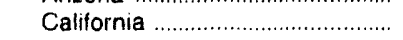 & 8,851 & 10,578 & 245,044 & 202,765 & 198,820 & 176,086 \\
\hline 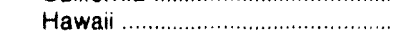 & 68 & 17 & 23,428 & 21,766 & 4,377 & 4,280 \\
\hline 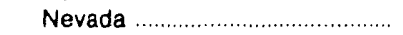 & 11,285 & 11,078 & 17,653 & 16,634 & 53,150 & 99,407 \\
\hline 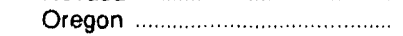 & 71,483 & 82,988 & 58,974 & 47,226 & 26,879 & 28,868 \\
\hline 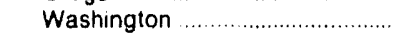 & 117,356 & 120,587 & 102,560 & 75,037 & 35,657 & 39,830 \\
\hline
\end{tabular}

See foolnotes at end of table. 
Table 4. Sales of Distillate Fuel Oil by End Use, 1988 and 1989-Continued (Thousand Gallons)

\begin{tabular}{|c|c|c|c|c|c|c|}
\hline \multirow{3}{*}{ Destination } & \multicolumn{2}{|c|}{ Oll Company } & \multicolumn{2}{|c|}{ Farm } & \multicolumn{2}{|c|}{ Electric Utility } \\
\hline & 1988 & 1989 & 1988 & 1989 & 1988 & 1989 \\
\hline & & & 1000 & 10000 & 1000 & \\
\hline U.S. Total & 622,736 & 683,683 & $3,162,575$ & $3,360,092$ & 720,137 & 887,599 \\
\hline 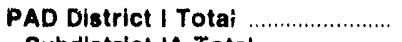 & 39,973 & 44,067 & 459,009 & 457,637 & 378,314 & 516,159 \\
\hline Subdistrict IA Total & 1,696 & 2,106 & 24,647 & 21,820 & 27,337 & 32,525 \\
\hline 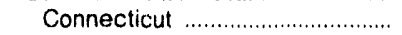 & 273 & 329 & 2,166 & 2,897 & 2,165 & 4,431 \\
\hline 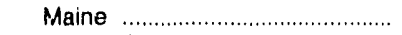 & 13 & 463 & 6.949 & 5,219 & 546 & 2,398 \\
\hline 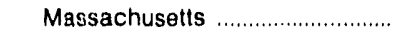 & 970 & 877 & 2.782 & 3,345 & 19,851 & 20,113 \\
\hline New Hampshire ............................. & 72 & 123 & 2,619 & 2,254 & 1,110 & 2,720 \\
\hline 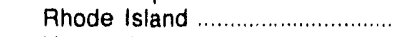 & 341 & 287 & 264 & 229 & 1,948 & 1,613 \\
\hline 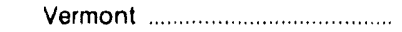 & 28 & 27 & 9,868 & 7,876 & 1,718 & 1,248 \\
\hline 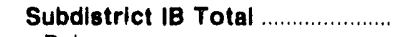 & 31,600 & 36,606 & 150,822 & 165,860 & 243,655 & 330,245 \\
\hline 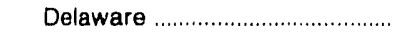 & 432 & 309 & 7,363 & 6,369 & 5,635 & 11,774 \\
\hline District of Columbia ..................... & - & - & - & - & 64,553 & 59,210 \\
\hline 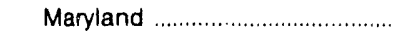 & 958 & 1,113 & 28,403 & 27,497 & 15,029 & 52,289 \\
\hline 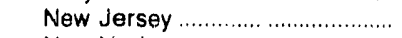 & 5,623 & 4,869 & 19,155 & 21,972 & 29,842 & 45,090 \\
\hline 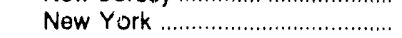 & 8.533 & 11,822 & 57,684 & 56,179 & 73,790 & 93,882 \\
\hline 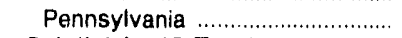 & 16,053 & 18,492 & 38,217 & 53,843 & 54,806 & 67,999 \\
\hline 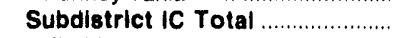 & 6,677 & 5,355 & 283,540 & 269,956 & 107,323 & 153,389 \\
\hline Florida & 1,066 & 1,125 & 94,897 & 54,642 & 50,454 & 65,510 \\
\hline 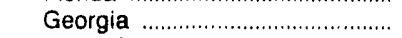 & 178 & 321 & 75,174 & 95,374 & 11,260 & 13,291 \\
\hline North Carolina .................................. & 25 & 93 & 49,807 & 45,504 & 16,592 & 18,444 \\
\hline South Carolina & 787 & 344 & 24,417 & 31,491 & 7,824 & 9,773 \\
\hline 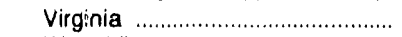 & 1,354 & 1,251 & 33,457 & 37,243 & 15,509 & 27,883 \\
\hline 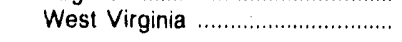 & 3,267 & 2,220 & 5,788 & 5,702 & 5,685 & 18,488 \\
\hline PAD District || Total ......................... & 60,905 & 72,953 & $1,595,064$ & $1,678,844$ & 144,946 & 154,963 \\
\hline 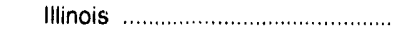 & 7,885 & 7,323 & 197,714 & 205,518 & 26,655 & 20,280 \\
\hline 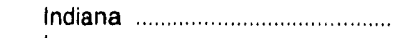 & 1,348 & 1,121 & 88,937 & 101,433 & 17.145 & 23,953 \\
\hline 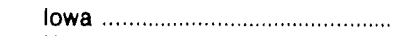 & 57 & 85 & 183,670 & 177,815 & 7.762 & 3,975 \\
\hline 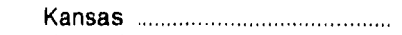 & 8,083 & 9,667 & 153,373 & 159,526 & 5,652 & 6,144 \\
\hline 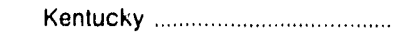 & 600 & 823 & 46,076 & 46,509 & 7,866 & 14,074 \\
\hline 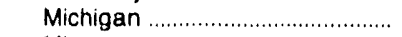 & 4,056 & 1,630 & 78,681 & 72,086 & 30,715 & 31,072 \\
\hline 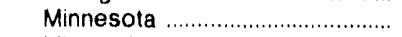 & 536 & 631 & 161,127 & 155,934 & 7,596 & 5,252 \\
\hline 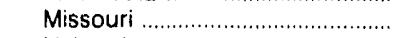 & 486 & 380 & 78,460 & 95,572 & 8,635 & 7,915 \\
\hline 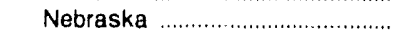 & 479 & 27 & 166,328 & 175,469 & 582 & 3,211 \\
\hline 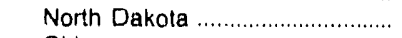 & 2,809 & 5,326 & 86,453 & 108,306 & 1,799 & 3,540 \\
\hline 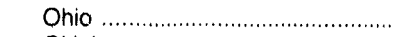 & 7,892 & 7,414 & 79.411 & 90,144 & 18,186 & 17,498 \\
\hline 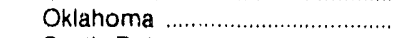 & 24,877 & 37,330 & 53,994 & 49,546 & 59 & 446 \\
\hline 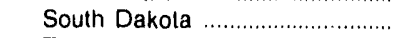 & 12 & 14 & 95,681 & 89,756 & 1,139 & 709 \\
\hline 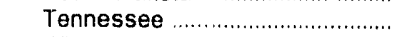 & 1,029 & 664 & 39,254 & 47,708 & 2,558 & 2,835 \\
\hline 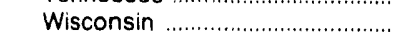 & 757 & 518 & 85,905 & 103.523 & 8,596 & 14,060 \\
\hline PAD District III Totai ........................ & 423,412 & 494,433 & 570,219 & 541,353 & 64,493 & 94,515 \\
\hline Alabama & 1,361 & 7,313 & 23,401 & 44,127 & 6,488 & 8,627 \\
\hline 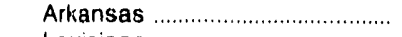 & 731 & 3,594 & 96,765 & 67,379 & 9,993 & 9,110 \\
\hline 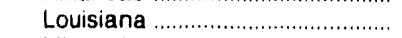 & 190,105 & 240,380 & 112,009 & 67,122 & 12,016 & 16,759 \\
\hline 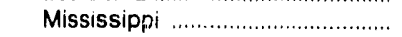 & 25,378 & 13,504 & 77,054 & 97,469 & 3,925 & 4,173 \\
\hline 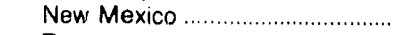 & 10,527 & 8,437 & $16,99 n$ & 12,381 & 348 & 5,225 \\
\hline 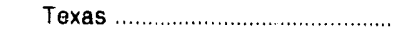 & 195,309 & 221,206 & 244,000 & 252,875 & 31,723 & 50,623 \\
\hline 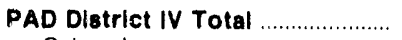 & 18,462 & 20,389 & 174,022 & 191,225 & 13,048 & 15,576 \\
\hline Colorado & 3,910 & 1,283 & 56,167 & 53,285 & 2,779 & 4,246 \\
\hline Idaho & 84 & 8 & 59,675 & 66,552 & 43 & 22 \\
\hline 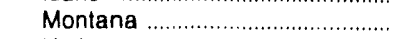 & 932 & 1,066 & 33,823 & 52,506 & 939 & 2,100 \\
\hline 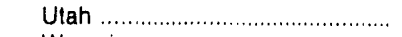 & 1,404 & 4,347 & 15,157 & 9,413 & 3,948 & 3,532 \\
\hline 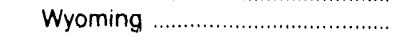 & 12,132 & 13,686 & 9,200 & 9,469 & 5,339 & 5,676 \\
\hline 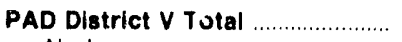 & 79,985 & 51,841 & 364,262 & 491,034 & 119,336 & 106,386 \\
\hline Alaska & 39,515 & 28,599 & 331 & 269 & 31,379 & 18,061 \\
\hline 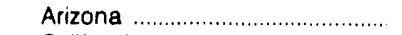 & 213 & 70 & 20,725 & 33,116 & 4,494 & 3,605 \\
\hline 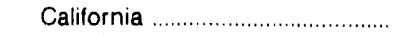 & 37,053 & 20,300 & 235,378 & 316,155 & 25,671 & 16,223 \\
\hline 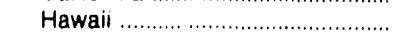 & 1,647 & 966 & 17,100 & 11,649 & 54,780 & 61,077 \\
\hline 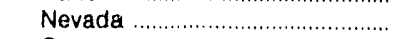 & 152 & 336 & 7,724 & 5,428 & 1,591 & 2,171 \\
\hline 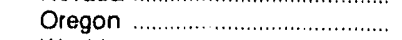 & 573 & 292 & 45,487 & 51,532 & 420 & 4,412 \\
\hline 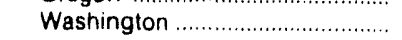 & 831 & 1,279 & 37,518 & 72,885 & 999 & 837 \\
\hline
\end{tabular}

See footnotes at end of table. 
Table 4. Sales of Distillate uel Oil by End Use, 1988 and 1989-Continued

(Thousand Gallons)

\begin{tabular}{|c|c|c|c|c|c|c|}
\hline \multirow[b]{2}{*}{ Destination } & \multicolumn{2}{|c|}{ Rallroad } & \multicolumn{2}{|c|}{ Vessel Bunkering } & \multicolumn{2}{|c|}{ On-Highway } \\
\hline & 1988 & 1989 & 1988 & 1989 & 1988 & 1989 \\
\hline U.S. Total & $3,095,736$ & $3,240,649$ & $1,991,773$ & $2,258,465$ & $20,038,691$ & $21,131,692$ \\
\hline PAD District I Total. & 614,324 & 551,268 & 534,979 & 521,110 & $6,712,224$ & $6,752,738$ \\
\hline Subidistrict IA Total ........................ & 17,960 & 35,062 & 52,628 & 67,894 & 836,650 & 827,953 \\
\hline 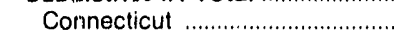 & 140 & 5,374 & 7,636 & 2,249 & 212,589 & 257,361 \\
\hline 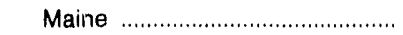 & 8,747 & 6,377 & 10,478 & 14,097 & 175,344 & 139,908 \\
\hline 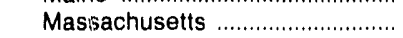 & 5,496 & 19,591 & 32,197 & 43,961 & 293,007 & 271,944 \\
\hline 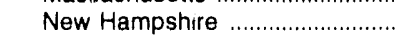 & 139 & 36 & 584 & 387 & 58,051 & 59,561 \\
\hline 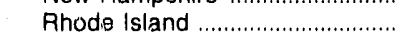 & 336 & 530 & 1,334 & 6,778 & 43,741 & 52,932 \\
\hline Verment & 3,102 & 3,154 & 400 & 422 & 53,918 & 46,250 \\
\hline 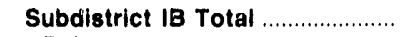 & 203,260 & 178,330 & 203,831 & 191,904 & $2,497,825$ & $2,571,926$ \\
\hline Delaware & 1,666 & 1,030 & 4,164 & 3,746 & 55,412 & 74,089 \\
\hline 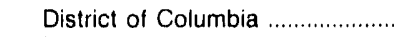 & 3,917 & 13,191 & 39 & 25 & 23,033 & 23,064 \\
\hline 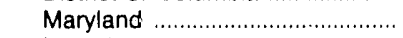 & 30,833 & 40,728 & 9,242 & 10,012 & 299,521 & 351,150 \\
\hline 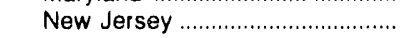 & 49,332 & 62,243 & 149,331 & 136,992 & 477,858 & 453,482 \\
\hline 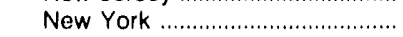 & 35,473 & 17,457 & 12,422 & 11,409 & 713,826 & 754,276 \\
\hline 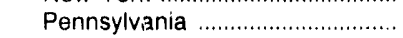 & 82,038 & 43,679 & 28,633 & 29,720 & 928,175 & 915,865 \\
\hline Subdistrici IC Total & 393,104 & 337,876 & 278,520 & 261,312 & $3,377,749$ & $3,352,856$ \\
\hline 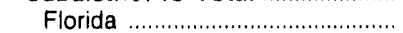 & 93,617 & 84,939 & 119,900 & 103,032 & 832,093 & 899,369 \\
\hline Georgia & 82,614 & 65,462 & 28,145 & 40,099 & 831,267 & 838,847 \\
\hline North Carolina & 52,122 & 36,898 & 9,806 & 9,930 & 711,035 & 614,485 \\
\hline South Carolina & 35,308 & 30,723 & 10,169 & 6,186 & 310,365 & 302,614 \\
\hline Virginia & 117,410 & 93,726 & $5 \ni, 569$ & 29,071 & 532,848 & 537,569 \\
\hline 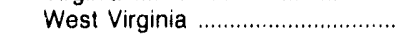 & 12,033 & 26,127 & 50,931 & 72,993 & 160,141 & 159,972 \\
\hline PAD District II Total ........................ & $1,231,924$ & $1,326,339$ & 308,552 & 458,790 & $6,645,800$ & $7,253,324$ \\
\hline 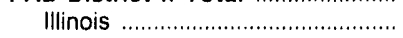 & 86,536 & 138,438 & 26,588 & 39,678 & 771,386 & 839,968 \\
\hline Indiana & 185,097 & 225,525 & 11,467 & 11,125 & 628,647 & 784,441 \\
\hline lowa & 35,603 & 33,695 & 1,673 & 2,896 & 333,931 & 349,044 \\
\hline Kansas & 143,757 & 168,700 & - & - & 331,926 & 303,893 \\
\hline 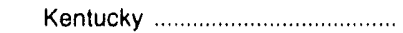 & 116,399 & 115,661 & 88,945 & 118,211 & 512,517 & 692,215 \\
\hline 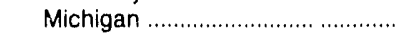 & 41,372 & 42,376 & 10,847 & 5,491 & 526,217 & 528,906 \\
\hline 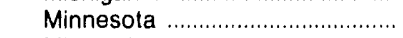 & 40,808 & 39,159 & 1,219 & 3,221 & 331,701 & 331,625 \\
\hline 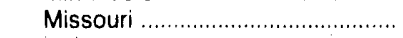 & 33,649 & 34,624 & 49,002 & 53,014 & $5: 35,424$ & 641,680 \\
\hline 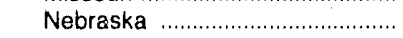 & 154,686 & 121,912 & - & 80 & 217,014 & 208,258 \\
\hline 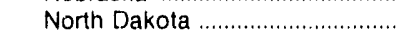 & 37,673 & 36,188 & - & - & 89,873 & 90,544 \\
\hline Ohio & 144,208 & 122,714 & 4,402 & $10, i 71$ & 861,428 & 9600,195 \\
\hline Oklahoma & 63,462 & 77,737 & 385 & 321 & 394,165 & 394,174 \\
\hline 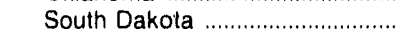 & 1,678 & 2,357 & - & - & 91,906 & 91,221 \\
\hline 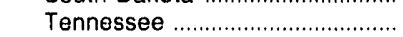 & 89,022 & 61,730 & 107,929 & 210,942 & 578,829 & 587,396 \\
\hline 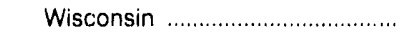 & 57,973 & 105,521 & 6,094 & 3,641 & 440,836 & 449,764 \\
\hline PAD Disirict III Total ........................ & 567,356 & 636,216 & 598,947 & 730,531 & $3,012,582$ & $3,221,237$ \\
\hline Alabama & 63,273 & 66,745 & 41,156 & 102,207 & 400,340 & 544,882 \\
\hline 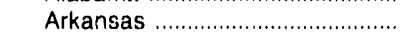 & 20,730 & 34,945 & 162 & 487 & 346,026 & 395,236 \\
\hline 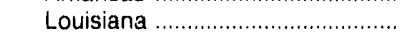 & 69,835 & 78,426 & 318,200 & 336,513 & 383,054 & 385,621 \\
\hline 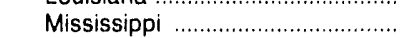 & $1,4,784$ & 17,760 & 66,241 & 55,534 & 302,361 & 302,523 \\
\hline 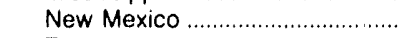 & 44,311 & 51,784 & - & - & $204,7: 14$ & 205,916 \\
\hline 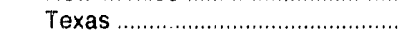 & 354,424 & 386,555 & 173,189 & 235,789 & $1,296,06 ; 7$ & $1,387,059$ \\
\hline PAD District IV Total ........................ & 285,616 & 310,192 & 73 & 131 & 725,429 & 734,904 \\
\hline 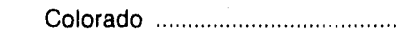 & 66,576 & 87.970 & - & 62 & 188,922 & 185,519 \\
\hline 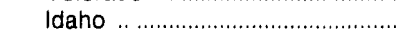 & 22,589 & 19,585 & 73 & 69 & 99,626 & 117,246 \\
\hline 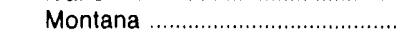 & 56,648 & 58,855 & - & - & 115,899 & 119,503 \\
\hline Utah & 33,422 & 34,590 & - & - & 149,108 & 125,184 \\
\hline 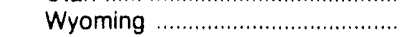 & 106,380 & 109,191 & - & - & 171,874 & 187,452 \\
\hline PAD District $\mathbf{V}$ Total $\ldots \ldots \ldots \ldots \ldots \ldots \ldots \ldots \ldots \ldots \ldots \ldots$ & 396,516 & 416,635 & 549,222 & 547,903 & $2,942,656$ & $3,169,489$ \\
\hline Alaska & 2,889 & 4,927 & 58,520 & 138.221 & 83,235 & 96,446 \\
\hline Arizona & 22,588 & 8,288 & - & - & 299,981 & 314,671 \\
\hline 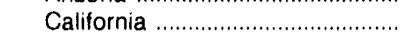 & 184,690 & 216,816 & 341,991 & 208,063 & $1,760,085$ & $1,913,647$ \\
\hline Hawaii & 18 & 17 & 55,937 & 86,679 & 21,935 & 23,717 \\
\hline 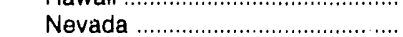 & 28,358 & 23,341 & $\begin{array}{r}0,001 \\
-\end{array}$ & 0 & 118,022 & 131,055 \\
\hline Oregon & 86,682 & 85,764 & 30,547 & 35,865 & 302,381 & 311,843 \\
\hline Washington & 71,230 & 77,481 & 62,226 & 79,074 & 357,017 & 378,110 \\
\hline
\end{tabular}

See footnotes at end of table. 
Table 4. Sales of Distillate Fuel Oil by End Use, 1988 and 1989-Continued (Thousand Gallons)

\begin{tabular}{|c|c|c|c|c|c|c|c|c|}
\hline \multirow[b]{2}{*}{ Destination } & \multicolumn{2}{|c|}{ Milltary } & \multicolumn{2}{|c|}{ Otf-Highway } & \multicolumn{2}{|c|}{ All Other } & \multicolumn{2}{|c|}{ Total } \\
\hline & $19 / 38$ & 1989 & 1988 & 1989 & 1988 & 1989 & 1988 & 1989 \\
\hline 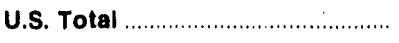 & 830,614 & 852,839 & $1,671,387$ & $1,689,651$ & 67,435 & 32,719 & $47,576,811$ & $49,788,896$ \\
\hline 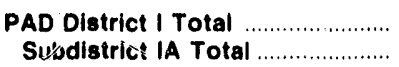 & $\begin{array}{r}203,769 \\
15,731\end{array}$ & $\begin{array}{r}162,510 \\
17,361\end{array}$ & $\begin{array}{r}471,882 \\
72,964\end{array}$ & $\begin{array}{r}530,679 \\
76,448\end{array}$ & $\begin{array}{r}17,975 \\
766\end{array}$ & $\begin{array}{r}12,998 \\
687\end{array}$ & $\begin{array}{r}19,973,062 \\
4,151,915\end{array}$ & $\begin{array}{r}20,279,614 \\
4,456,252\end{array}$ \\
\hline 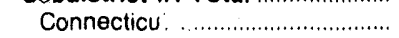 & 1,483 & 702 & 13,653 & 21,512 & 319 & 551 & $1,059,052$ & $1,192,619$ \\
\hline 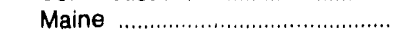 & 2,619 & 3,431 & 22,770 & 14,755 & - & 45 & 627,050 & 570,529 \\
\hline 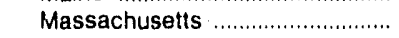 & 4,377 & 2,285 & 20,040 & 20,943 & 75 & - & $1,660,522$ & $1,845,647$ \\
\hline New Hampshire .............................. & 86 & 1,772 & 6,947 & 8,821 & - & - & $3 \uparrow 4,984$ & 355,744 \\
\hline 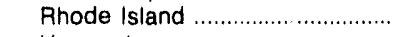 & 6,460 & 8,907 & 1,136 & 3,844 & 210 & 91 & 277,252 & 276,865 \\
\hline 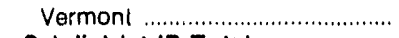 & 705 & 264 & 8,417 & 6,572 & 162 & - & 213,055 & 214,849 \\
\hline 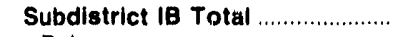 & 41,008 & 63,949 & 153,090 & 208,065 & 3,735 & 3,838 & $9,309,668$ & $9,580,703$ \\
\hline 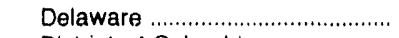 & 37 & 98 & 3,847 & 4.830 & 107 & - & 173,344 & 190,686 \\
\hline District of Columbia .................... & 10,046 & 3,444 & 92 & 36 & - & - & 141,939 & 132,181 \\
\hline 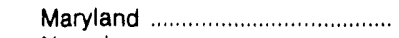 & 4,468 & 25,677 & 17,362 & 36,975 & 3,081 & 2,954 & 812,606 & 926,298 \\
\hline New Jersey .................................... & 6,830 & 10,489 & 32,246 & 60,297 & 36 & 55 & $2,077,454$ & $2,039,025$ \\
\hline 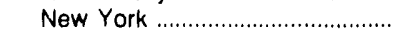 & 14,337 & 11,967 & 47,707 & 46,325 & 298 & 4 & $3,468,143$ & $3,500,894$ \\
\hline 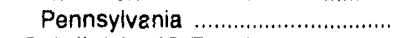 & 5,290 & 12,274 & 51,836 & 59,601 & 213 & 825 & $2,636,180$ & $2,791,619$ \\
\hline 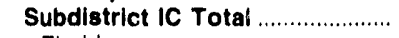 & 147,030 & 81,200 & 245,828 & 246,166 & 13,474 & 8,474 & $6,511,480$ & $6,242,660$ \\
\hline 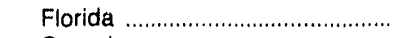 & 32,711 & 23,342 & 63,841 & 75,442 & 1,693 & 1,503 & $1,511,962$ & $1,506,480$ \\
\hline 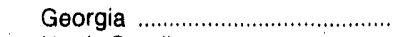 & 13,739 & 10,522 & 40,242 & 43,889 & 4,735 & 1,851 & $1,199,080$ & $1,219,807$ \\
\hline 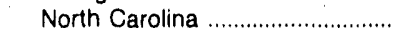 & 4,898 & 8,960 & 32,337 & 30,641 & 3,161 & 2,568 & $1,393,036$ & $1,200,013$ \\
\hline South Carolina & 3,662 & 2,939 & 28,340 & 30,580 & 1,452 & 1,044 & 550,915 & 550,822 \\
\hline Virginia & 91,454 & 35,251 & 60,282 & 42,212 & 2,401 & 1,493 & $1,459,236$ & $1,307,754$ \\
\hline West Virginia ................................ & 566 & 187 & 20,787 & 23,402 & 32 & 15 & 397,251 & 457,784 \\
\hline PAD Dlstrict II Total ........................ & 53,071 & 53,700 & $43 \%, 175$ & 410,067 & 42,684 & 16,777 & $13,392,376$ & $14,318,165$ \\
\hline 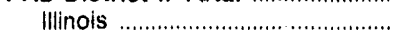 & 1,965 & 5,337 & 59,218 & 45,245 & 3,706 & 194 & $1,436,477$ & $1,544,425$ \\
\hline 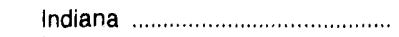 & 4,215 & 7,914 & 18,775 & 35,500 & 3,344 & 3,397 & $1,215,244$ & $1,472,196$ \\
\hline 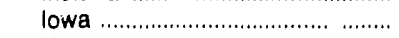 & 115 & 327 & 18,128 & 11,561 & 2,979 & 2,260 & 683,668 & 676,545 \\
\hline 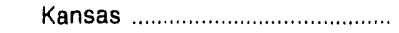 & 2,036 & 1,030 & 17,910 & 14,882 & 1,587 & 1,928 & 697,397 & $696,79 ?$ \\
\hline 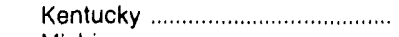 & 11,286 & 6,529 & 22,248 & 26,279 & 752 & 23 & $1,048,070$ & $1,259,451$ \\
\hline 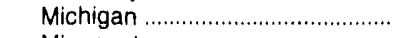 & $3,3 \% 0$ & 3,791 & 51,988 & 54,695 & 2,292 & 2,101 & $1,193,926$ & $1,148,997$ \\
\hline 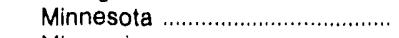 & 2,828 & 2,445 & 24,719 & 31,523 & 2,219 & 2,190 & 856,779 & 896,000 \\
\hline 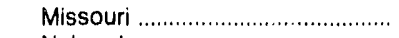 & 6,597 & 6,017 & 38,816 & 25,902 & 7,101 & 1,403 & 899,422 & 977,602 \\
\hline 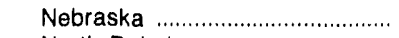 & 1,489 & 1,281 & 18,921 & 20,582 & - & - & 586,714 & 561,016 \\
\hline 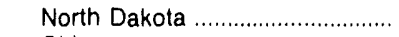 & 2,620 & 1,620 & 5,720 & 5,433 & 80 & 234 & 289,388 & 328,260 \\
\hline Ohio & 9,418 & 10,358 & 29,020 & 33,691 & 374 & 404 & $1,568,092$ & $1,703,534$ \\
\hline 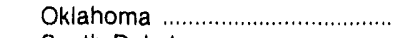 & 2,522 & 1,796 & 29,393 & 26,563 & 4,827 & 274 & 628,127 & 647,317 \\
\hline South Dakota & 763 & 616 & 12,218 & 6,798 & 352 & 651 & 268,091 & 255,015 \\
\hline 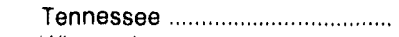 & 1,102 & 3,355 & 62,679 & 32,072 & 1,292 & 783 & 986,638 & $1,031,648$ \\
\hline 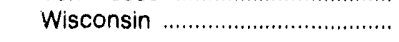 & 2,746 & 1,282 & 23,422 & 39,341 & 11,779 & 935 & $1,034,3<1$ & $1,119.367$ \\
\hline PAD District III Total ........................ & 256,918 & 323,472 & 318,345 & 275,769 & 5,534 & 2,505 & $6,343,873$ & $6,877,617$ \\
\hline Alabama & 3,059 & 4,634 & 33,478 & 46,724 & 2,255 & 1,463 & 741,394 & 924,936 \\
\hline 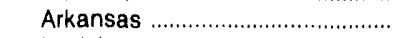 & 1,555 & 1,514 & 19,505 & 23,503 & 1,760 & 46 & 516,794 & 566,118 \\
\hline 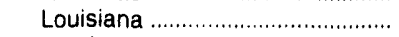 & 13,209 & 60,773 & 47,184 & 40,216 & 188 & 201 & $1,203,045$ & $1,269,939$ \\
\hline 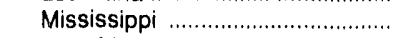 & 73,503 & 53,723 & 21,278 & 24,562 & 554 & 118 & 622,574 & 613,082 \\
\hline 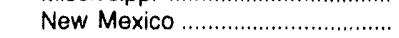 & 1,186 & 667 & 22,168 & 15,716 & 0 & 35 & 360,304 & 348,395 \\
\hline 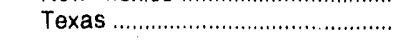 & 164,407 & 202,160 & 174,734 & $1,5,047$ & 776 & 643 & $2,899,763$ & $3,155,148$ \\
\hline PAD District IV Total ........................ & 14,074 & 13,402 & 103,898 & 91,261 & 370 & 438 & $1,629,278$ & $1,692,305$ \\
\hline Colorado & 7,660 & 8,144 & 39,012 & 26,568 & 29 & 45 & 446,506 & 425,360 \\
\hline 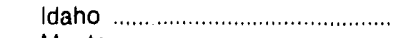 & 2,295 & 720 & 15,356 & 13,524 & - & 25 & 257,514 & 284,182 \\
\hline 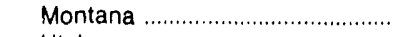 & 454 & 342 & 10,089 & 15,587 & 192 & 24 & 251,770 & 318,045 \\
\hline 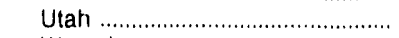 & 3,430 & 4,016 & 19,222 & 18,086 & - & - & 294,032 & 256,759 \\
\hline 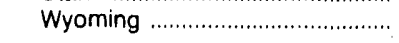 & 255 & 181 & 20,219 & 17,396 & 149 & 344 & 379,456 & 407,959 \\
\hline PAD District V Total ........................ & 302,781 & 299,754 & 344,087 & 381,676 & 871 & - & $6,238,223$ & $\mathbf{5 , 6 2 1 , 1 9 5}$ \\
\hline Alcska & 16,224 & 35,657 & 12,800 & 14,630 & 37 & - & 354,283 & 476,883 \\
\hline 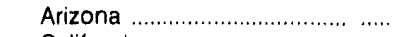 & 1,223 & 2,358 & 23,360 & 39,741 & - & - & 426,155 & 480,906 \\
\hline 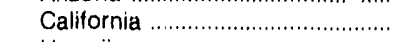 & 204,777 & 215,013 & 192,850 & 202,235 & 749 & - & $3,435,959$ & $3,497,880$ \\
\hline 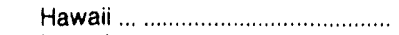 & 50,511 & 32,595 & 6,677 & 5,680 & - & - & 236,478 & 248,444 \\
\hline 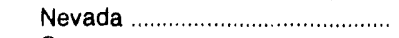 & 4,553 & 4,605 & 30,296 & 25,183 & 31 & - & 272,814 & 319,239 \\
\hline Oregon & 1,284 & 767 & 40,108 & 46,647 & - & - & 664,819 & 696,206 \\
\hline 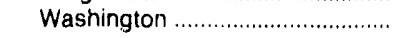 & 24,210 & 8,758 & 37,995 & 47,759 & 55 & - & 847,714 & 901,637 \\
\hline
\end{tabular}

Dashes $(-)$.: No dita reported

Note: Totals may not equal sum of components due to independent rounding.

Sources: - Energy Intormation Administration Form EIA-821, "Annual Fuel Oil and Kerosene Sales Report," for 1988 and 1989

- On-Highway Diese! data are Federal Highway Administation statistics of highway spevial fuels use. 
Table 5. Sales of Residual Fuel Oil by End Use, 1988 and 1989 (Thousand Gallons)

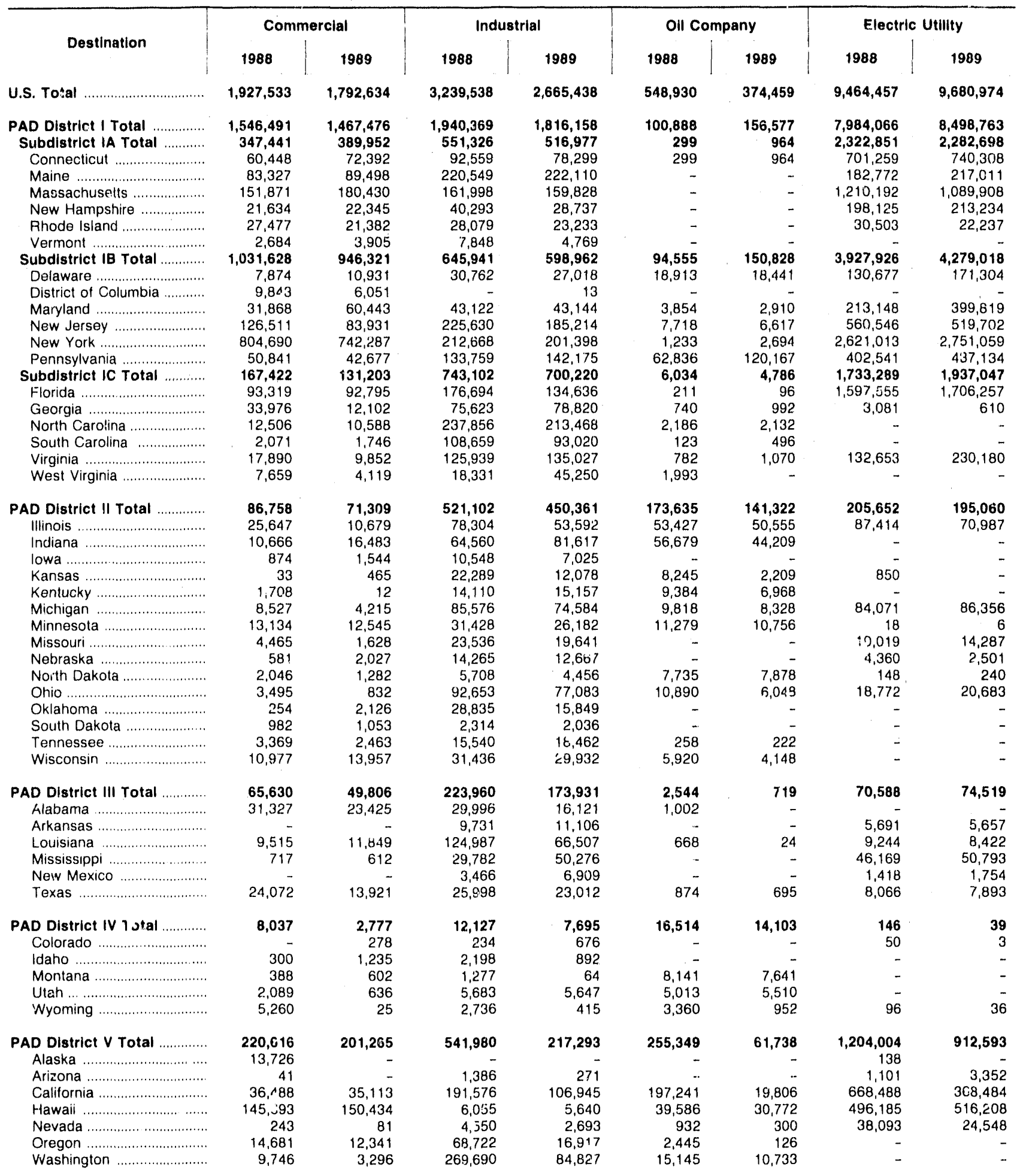

See fuotnotes at end of table. 
Table 5. Sales of Residual Fuel Oil by End Use, 1988 and 1989-Continued

(Thousand Gallons)

\begin{tabular}{|c|c|c|c|c|c|c|c|c|c|c|}
\hline \multirow{2}{*}{ Destination } & \multicolumn{2}{|c|}{ Rallroad } & \multicolumn{2}{|c|}{ Vessel Bunkering } & \multicolumn{2}{|c|}{ Military } & \multicolumn{2}{|c|}{ All Other } & \multicolumn{2}{|c|}{ Total } \\
\hline & 1988 & 1989 & 1988 & 1989 & 1988 & 1989 & 1988 & 1989 & 1988 & 1989 \\
\hline U.S. Total ........... & 32 & 39 & $6,354,536$ & $7,191,689$ & 125,318 & 92,229 & 58,880 & 31,838 & $21,719,226$ & $21,829,300$ \\
\hline PAD District I Total ................. & - & - & $1,410,904$ & $1,381,741$ & 96,270 & 79,609 & 4,719 & 208 & $13,083,707$ & $13,400,532$ \\
\hline Subdistrict IA Total ............... & - & - & 51,996 & 49,484 & 21,752 & 22,627 & - & - & $3,295,666$ & $3,262,701$ \\
\hline 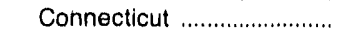 & - & - & 1,325 & 341 & 4,495 & 2,714 & - & - & 860,385 & 895,018 \\
\hline 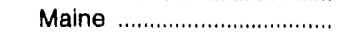 & - & - & 18,545 & 5,729 & - & 3,580 & - & - & 505,193 & 537,928 \\
\hline 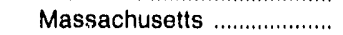 & - & - & 24,651 & 42,742 & 5,281 & 12,607 & - & - & $1,553,993$ & $1,485,516$ \\
\hline 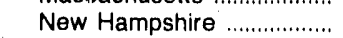 & - & - & 672 & - & 5,808 & 919 & - & - & 266,532 & 265,236 \\
\hline 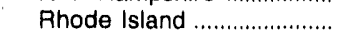 & - & - & 6,803 & 671 & 6,169 & 2,497 & - & - & 99,031 & 70,020 \\
\hline 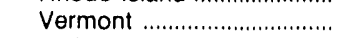 & - & - & - & - & - & 310 & - & - & 10,533 & 8,984 \\
\hline Subdistrict IB Total ................ & - & - & 829,599 & 758,807 & 43,317 & 35,854 & 4,205 & - & $6,577,171$ & $6,769,789$ \\
\hline 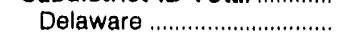 & - & - & 38,735 & 41,561 & - & - & - & - & 226,961 & 269,255 \\
\hline District of Columbia ............ & - & - & - & - & 455 & 1,853 & - & - & 10,298 & 7,917 \\
\hline Maryland ................................. & - & - & 94,942 & 92,725 & 21,592 & 20,756 & - & - & 408,527 & 619,797 \\
\hline 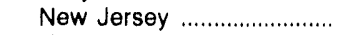 & - & - & 332,802 & 417,851 & 6,317 & 2,534 & 86 & - & $1,259,611$ & $1,215,849$ \\
\hline 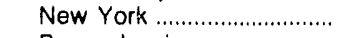 & - & - & 123,456 & 17,095 & 12,143 & 7,724 & 4,119 & - & $3,779,323$ & $3,722,257$ \\
\hline Pennsylvania ......................... & - & - & 239,664 & 189,574 & 2,810 & 2,986 & - & - & 892,451 & 934,713 \\
\hline Subdistrict IC Total ........... & - & - & 529,309 & 573,450 & 31,200 & 21,128 & 513 & 208 & $3,210,870$ & $3,368,042$ \\
\hline Florida & - & - & 355,522 & 372,112 & 8,650 & 6,537 & 246 & - & $2,232,197$ & $2,312,433$ \\
\hline 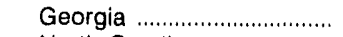 & - & - & 18,274 & 29,423 & 1,628 & 1,706 & - & - & 133,323 & 123,654 \\
\hline North Carolina ........................ & - & - & 8,913 & 27,619 & 9,504 & 3,693 & 267 & 208 & 271,232 & 257,706 \\
\hline 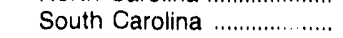 & - & - & 29,307 & 25,874 & 4,892 & 5,558 & - & - & 145,052 & 126,694 \\
\hline 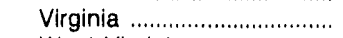 & - & - & 117,293 & 118,421 & 6,526 & 3,635 & - & - & 401,083 & 498,185 \\
\hline West Virginia ............................ & - & - & - & - & - & - & - & - & 27,982 & 49,369 \\
\hline PAD District $\|$ Total .............. & 32 & 1 & 24,593 & 18,710 & 4,492 & 3,760 & 240 & 464 & $1,016,504$ & 880,987 \\
\hline 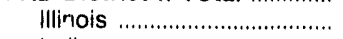 & - & - & 15,351 & 2,671 & 142 & - & 39 & - & 260,322 & 188,483 \\
\hline 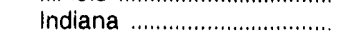 & - & - & 6,831 & 9,908 & - & - & - & - & 138,736 & 152,217 \\
\hline 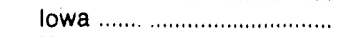 & - & 1 & - & - & - & 8 & - & - & 11,423 & 8,578 \\
\hline 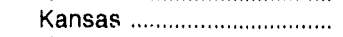 & - & - & - & - & - & - & - & - & 31,419 & 14,752 \\
\hline 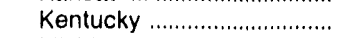 & - & - & - & - & - & - & - & - & 25,202 & 22,137 \\
\hline 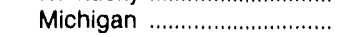 & - & - & 1,315 & 5,397 & - & - & - & 62 & 189,307 & 178,943 \\
\hline 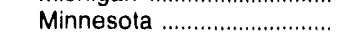 & 11 & - & 306 & 73 & - & - & 185 & 130 & 56,361 & 49,692 \\
\hline 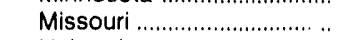 & 21 & - & - & - & 3,850 & 3,250 & - & - & 41,891 & 38,806 \\
\hline 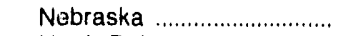 & - & - & - & - & - & - & 16 & 16 & 19,221 & 17,211 \\
\hline North Dakota ......................... & - & - & - & - & - & - & - & 256 & 15,637 & 14,111 \\
\hline Ohio & - & - & 524 & 451 & - & - & - & - & 126,333 & 105,097 \\
\hline 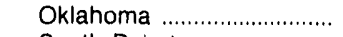 & - & - & - & - & - & - & - & - & 29,089 & 17,974 \\
\hline South Dahota ......................... & - & - & - & - & - & 2 & - & - & 3,297 & 3,091 \\
\hline 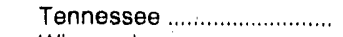 & - & - & 66 & 22 & 500 & 500 & - & - & 19,734 & 21,669 \\
\hline 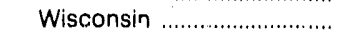 & - & - & 200 & 187 & .. & - & - & - & 48,533 & 48,224 \\
\hline PAD District III Total ............. & - & - & $2,167,731$ & $2,535,626$ & 3,918 & 1,077 & 20,886 & - & $2,555,259$ & $2,835,679$ \\
\hline 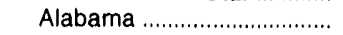 & - & - & 90,239 & 130,922 & 26 & 1,077 & - & - & 152,590 & 171,545 \\
\hline 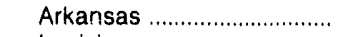 & - & - & - & - & - & - & - & - & 15,422 & 16,763 \\
\hline 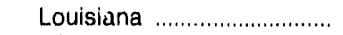 & - & - & $1,024,105$ & $1,130,135$ & 3,892 & .. & 1,656 & - & $1,174,067$ & $1,216,936$ \\
\hline 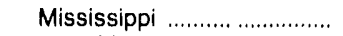 & - & - & 77,565 & 56,303 & - & - & - & - & 154,233 & 157,984 \\
\hline New Mexico ............................... & - & - & - & - & - & - & - & - & 4,885 & 8,663 \\
\hline 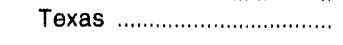 & - & - & 975,822 & $1,218,266$ & - & - & 19,230 & - & $1,054,063$ & $1,263,788$ \\
\hline PAD District IV Total .............. & - & 19 & - & - & - & - & 40 & 225 & 36,865 & 24,858 \\
\hline 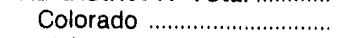 & - & - & - & - & - & - & - & - & 284 & 957 \\
\hline 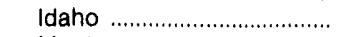 & - & - & - & - & - & - & - & - & 2,498 & 2,127 \\
\hline 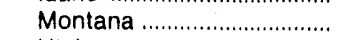 & - & - & - & - & - & - & - & 197 & 9,806 & 8,504 \\
\hline 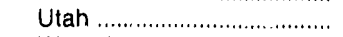 & - & 9 & - & - & - & - & - & - & 12,785 & 11,802 \\
\hline Wyoming & - & 11 & - & - & - & - & 40 & 28 & 11,492 & 1,467 \\
\hline PAD District V Total .............. & - & 19 & $2,151,30 \%$ & $3,255,612$ & 20,639 & 7,783 & 32,996 & 30,942 & $5,026,891$ & $4,687,244$ \\
\hline 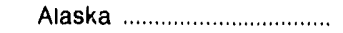 & - & - & 6,199 & 530 & - & - & - & - & 20,063 & 5,530 \\
\hline 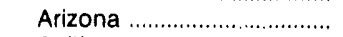 & - & - & - & - & - & - & - & - & 2,528 & 3,623 \\
\hline 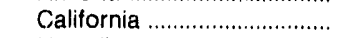 & - & - & $2,070,421$ & $2,297,366$ & 6,538 & - & - & - & $3,170,751$ & $2,827,714$ \\
\hline 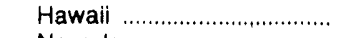 & - & - & $\{9,524$ & 101,297 & 2,913 & 3,196 & $3 \% 748$ & 30,877 & 792.704 & 838,424 \\
\hline 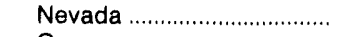 & - & - & - & - & - & - & - & - & 43,818 & 27,622 \\
\hline 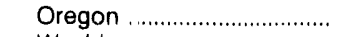 & - & 19 & 186,730 & 222,279 & 215 & - & 36 & 65 & 272,829 & 251,747 \\
\hline 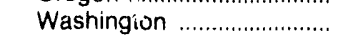 & - & - & 418,433 & 629,140 & 10,973 & 4,587 & 211 & - & 724,198 & 732,584 \\
\hline
\end{tabular}

Dashies $(\cdot)=$ No data reported.

Note: Totals may not equal surn of components due to independent rounding.

Sources: - Energy information Administration Form ElA-821, "Annual Fuel Oil and Kerosene Sales Report," for 1988 ar,d 1989. 
Table 6. Sales of Kerosene by End Use, 1988 and 1989

(Thousand Gallons)

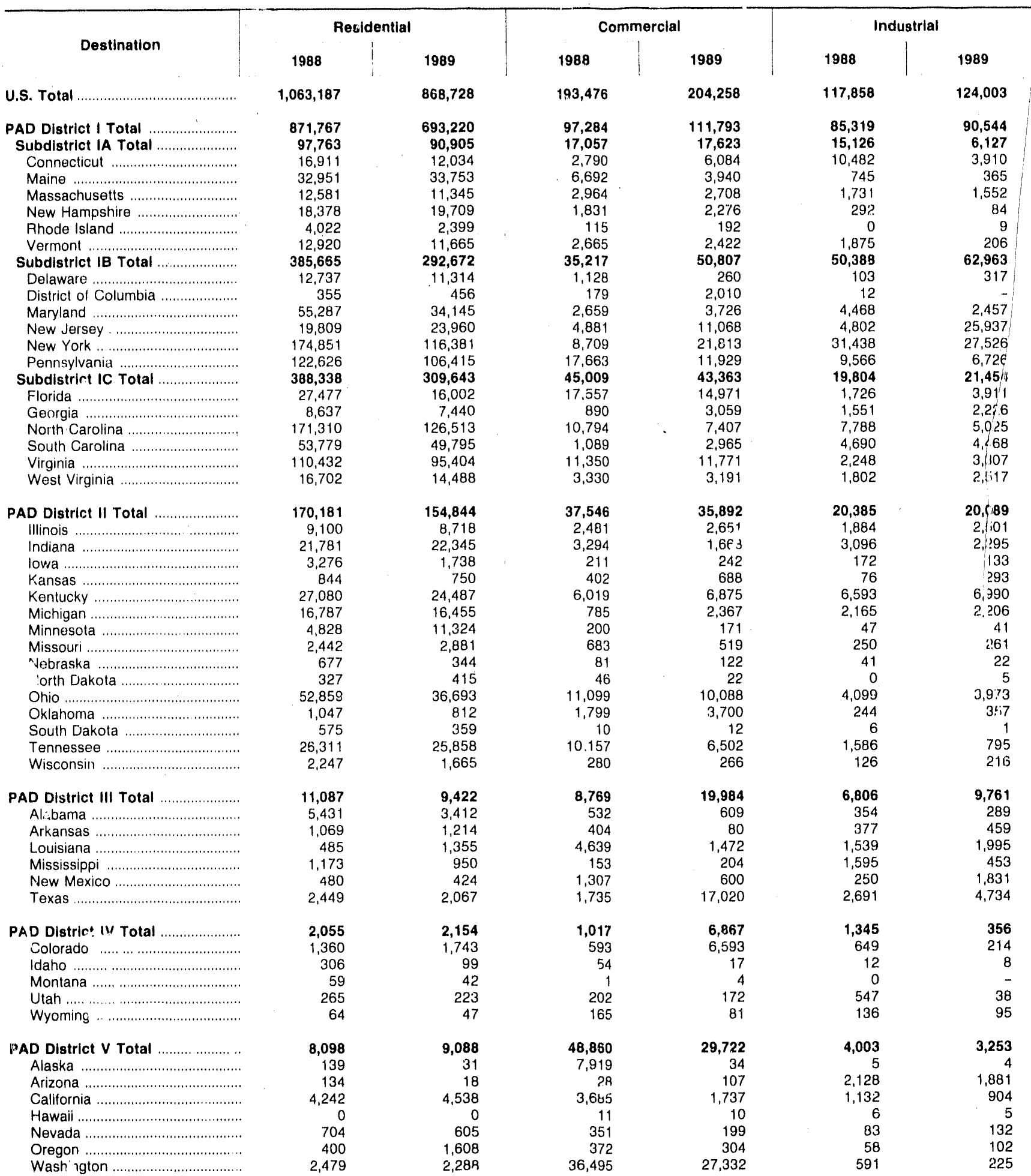

See footnotes at end of table. 
Table 6. Sales of Kerosene by End Use, 1988 and 1989-Continued

(Thousand Gallons)

\begin{tabular}{|c|c|c|c|c|c|c|}
\hline & & & & & & \\
\hline Destination & & & & & & \\
\hline & 1988 & 1989 & 1988 & 1989 & 1988 & 1989 \\
\hline U.S. Total & 33,426 & 30,081 & 70,370 & 64,598 & $1,478,316$ & $1,291,668$ \\
\hline PAI District I Total ......................... &,- 659 & 16,361 & 51,824 & 49,553 & $1,126,852$ & 961,472 \\
\hline 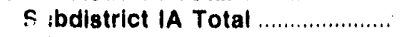 & 2,462 & 2,146 & 2,153 & 8,001 & 134,561 & 124,802 \\
\hline 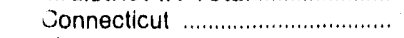 & 22 & 10 & 202 & 6,137 & 30,407 & 28,176 \\
\hline Maine & 791 & 1,144 & 792 & 530 & 41,970 & 39,732 \\
\hline 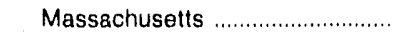 & 11 & 89 & 288 & 768 & 17,575 & 16,461 \\
\hline New Hampshire ........................... & 58 & 54 & 99 & 457 & 20,658 & 22,581 \\
\hline 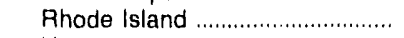 & 1 & 1 & 692 & 36 & 4,830 & 2,638 \\
\hline 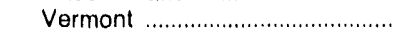 & 1,580 & 848 & 80 & 72 & 19,120 & 15,213 \\
\hline Subdistrict iB Total & 6,693 & 8,711 & 35,221 & 36,555 & 513,185 & 451,707 \\
\hline Delaware & 382 & 16 & 4 & 35 & 14,353 & 11,943 \\
\hline District of Columbia ..................... & - & - & 63 & - & 608 & 2,465 \\
\hline 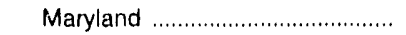 & 363 & 1,743 & 1,302 & 189 & 64,078 & 42,261 \\
\hline 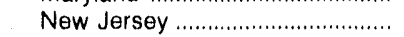 & 32 & 7 & 28,453 & 3,584 & 57,977 & 64,557 \\
\hline 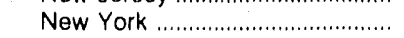 & 4,079 & 4,791 & 1,983 & 30,988 & 221,061 & 201,500 \\
\hline 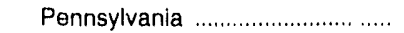 & 1,836 & 2,153 & 3,416 & 1,758 & 155,107 & 128,982 \\
\hline Subdistrict IC Total .......................... & 11,505 & 5,504 & 14,450 & 4,998 & 479,106 & 384,963 \\
\hline 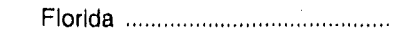 & 54 & 73 & 1,629 & 842 & 48,443 & 35,799 \\
\hline 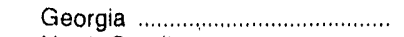 & 1,883 & 55 & 1,184 & 409 & 14,147 & 13,189 \\
\hline 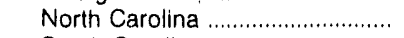 & 5,667 & 1,787 & 6,164 & 898 & 201,723 & 141,630 \\
\hline 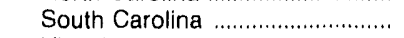 & 1,001 & 1,238 & 1,756 & 1,428 & 62,315 & 59,894 \\
\hline Virginia & 2,411 & 1,500 & 1,599 & 1,077 & 128,040 & 113,059 \\
\hline 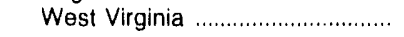 & 488 & 852 & 2,117 & 345 & 24,439 & 21,392 \\
\hline PAD District II Total ......................... & 9,917 & 10,724 & 10,770 & 5,842 & 248,799 & 227,389 \\
\hline 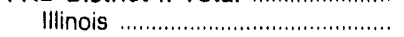 & 843 & 536 & 404 & 999 & 14,711 & 15,405 \\
\hline Indiana & 748 & 830 & 978 & 169 & 29,896 & 27,307 \\
\hline lowa & 725 & 552 & 100 & 313 & 4,484 & 2,978 \\
\hline 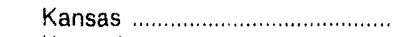 & 320 & 490 & 115 & 124 & 1,758 & 2,345 \\
\hline 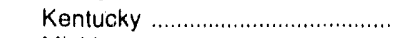 & 1,185 & 871 & 146 & 388 & 41,022 & 39,611 \\
\hline Michigan ....................................... & 178 & 295 & 13 & 379 & 19,926 & 21,703 \\
\hline 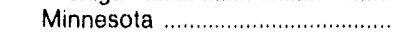 & 1,215 & 1,998 & 149 & 58 & 6,439 & 13,592 \\
\hline 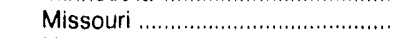 & 180 & 381 & 588 & 742 & 4,143 & 4,784 \\
\hline 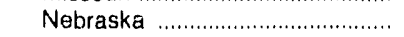 & 353 & 417 & 2,037 & 11 & 3,109 & 915 \\
\hline 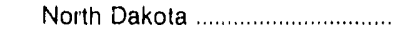 & 86 & 15 & 164 & 11 & 623 & 468 \\
\hline 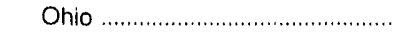 & 2,978 & 3,235 & 2,162 & 2,151 & 73,197 & 56,140 \\
\hline 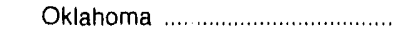 & 433 & 93 & 194 & 60 & 3,716 & $5,02 ?$ \\
\hline 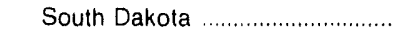 & 180 & 198 & 41 & 33 & 811 & $6 \mathrm{C} 2$ \\
\hline 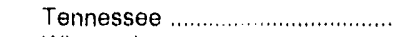 & 318 & 404 & 3,592 & 330 & 41,964 & 33,890 \\
\hline 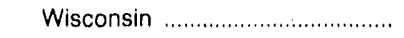 & 175 & 407 & 90 & 73 & 2,918 & 2,627 \\
\hline PAD District Iil Total ........................ & 1,142 & 1,552 & 7,338 & 8,233 & 35,442 & 48,952 \\
\hline 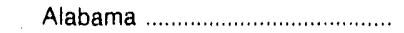 & 108 & 27 & 420 & 412 & 6,845 & 4,748 \\
\hline 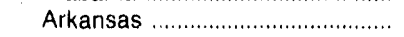 & 75 & 135 & 216 & 66 & 2,142 & 1.954 \\
\hline 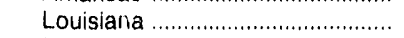 & 46 & 352 & 4,120 & 1,884 & 10,829 & 7,057 \\
\hline 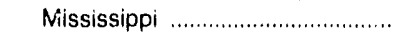 & 771 & 439 & 10 & 673 & 3,702 & 2,719 \\
\hline New Mexico ....................................... & 21 & 41 & 73 & 64 & 2,130 & 2,960 \\
\hline 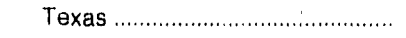 & 421 & 558 & 2,499 & 5,135 & 9,794 & 29,514 \\
\hline PAD District IV Totul ........................ & 1,159 & 781 & 69 & 360 & 5,645 & 10,517 \\
\hline 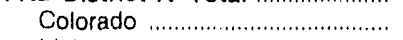 & 969 & 622 & 13 & 329 & 3,585 & 9,501 \\
\hline Idaho & 26 & 22 & 4 & 5 & 402 & 151 \\
\hline 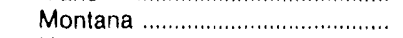 & 102 & 91 & 0 & 4 & 163 & 142 \\
\hline Utah & 37 & 23 & 17 & 10 & 1,069 & 466 \\
\hline 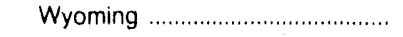 & 25 & 22 & 35 & 12 & 427 & 257 \\
\hline 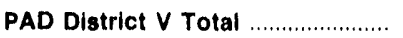 & 248 & 664 & 369 & 610 & 61,578 & 43,337 \\
\hline Alaska & - & - & 13 & 12 & 8,076 & 81 \\
\hline 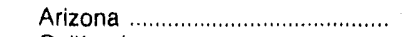 & 9 & 17 & 42 & 63 & 2,340 & 2,086 \\
\hline 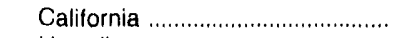 & 112 & 501 & 291 & 392 & 9,461 & 8,072 \\
\hline 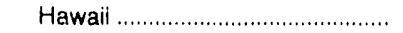 & 0 & 0 & - &.. & 17 & 16 \\
\hline 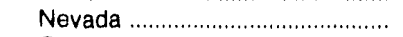 & 21 & 43 & 10 & 116 & 1,170 & 1,095 \\
\hline 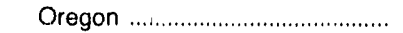 & 6 & 53 & 8 & 17 & 844 & 2,083 \\
\hline Washington & 99 & 50 & 5 & 9 & 39,670 & 29,903 \\
\hline
\end{tabular}

Dashes $(-)=$ No data reported.

Note: Totals may riot equal sum of components due to independent rounding.

Sources: - Energy Information Administration Form ElA-821. "Annual Fuel Oil and Kerosene Sales Report," for 1988 and 1989.

- Kerosene data are based on data from the Energy Iriformation Administration's "Petroleum Supply Annual," Volume 1, 1988 and 1989, (DOE/EIA. $0340(88) / 1 \cdot(89) / 1)$. 
Table 7. Sales for Residential Use: Distillate Fuel Oil and Kerosene, 1989 (Thousand Gallons)

\begin{tabular}{|c|c|c|c|c|}
\hline \multirow{2}{*}{ Destination } & \multicolumn{3}{|c|}{ Distillate Fuel Oll } & \multirow{2}{*}{ Kerosene } \\
\hline & No. 1 & No. 2 & Total & \\
\hline 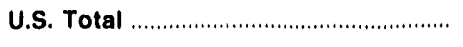 & 324,076 & $8,324,553$ & $8,648,629$ & 868,728 \\
\hline $\begin{array}{l}\text { PAD District I Total } \\
\text { Subdistrict IA Total }\end{array}$ & $\begin{array}{l}45,910 \\
12,278\end{array}$ & $\begin{array}{l}6,855,779 \\
2,405,608\end{array}$ & $\begin{array}{l}6,901,689 \\
2,417,886\end{array}$ & $\begin{array}{r}693,220 \\
90,905\end{array}$ \\
\hline 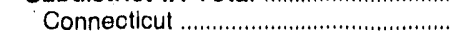 & 1,034 & 685,801 & 686,835 & 12,034 \\
\hline 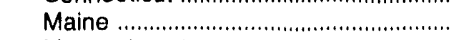 & 7,804 & 266,303 & 274,107 & 33,753 \\
\hline 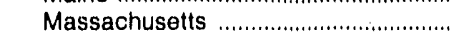 & 470 & 990,640 & 991,111 & 11,345 \\
\hline 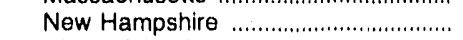 & 1,210 & 206,754 & 207,964 & 19.709 \\
\hline 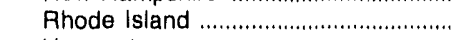 & 1,386 & 152,055 & 153,441 & 2,399 \\
\hline 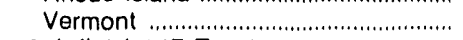 & 375 & 104,055 & 104,429 & 11,665 \\
\hline 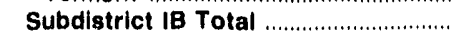 & 17,325 & $3,813,929$ & $3,831,255$ & 292,672 \\
\hline 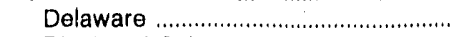 & 149 & 63,636 & 63,785 & 11,314 \\
\hline District of Columbia .................................. & - & 7,029 & 7,029 & 456 \\
\hline 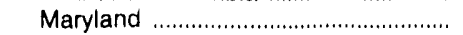 & 1,049 & 247,029 & 248,079 & 34,145 \\
\hline 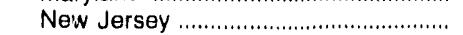 & 51 & 768,760 & 768,811 & 23,960 \\
\hline 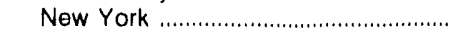 & 8,814 & $1,670,512$ & $1,679,326$ & 116,381 \\
\hline 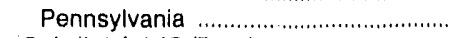 & 7,762 & $1,056,963$ & $1,064,225$ & 106,415 \\
\hline 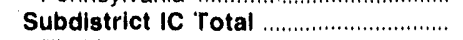 & 16,307 & 636,242 & 652,549 & 309,643 \\
\hline Florida & 478 & 17,039 & 17,517 & 16,002 \\
\hline 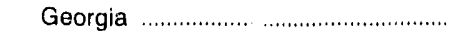 & 70 & 12,813 & 12,884 & 7,440 \\
\hline 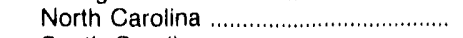 & 8,113 & 217,629 & 225,742 & 126,513 \\
\hline 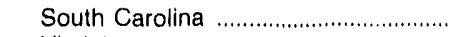 & 2,034 & 59,835 & 61,869 & 49,795 \\
\hline 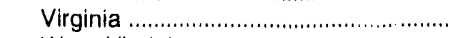 & 4,187 & 299,248 & 303,435 & 95,404 \\
\hline West Virginia ......................................... & 1,425 & 29,678 & 31,102 & 14,488 \\
\hline 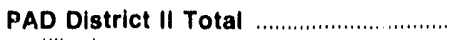 & 227,232 & $1,176.837$ & $1,404,069$ & 154,844 \\
\hline 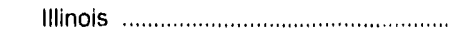 & 12,595 & 67,378 & 79,973 & 8,718 \\
\hline 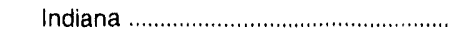 & 24,433 & 92,628 & 117,061 & 22,345 \\
\hline 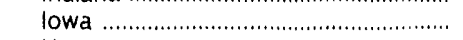 & 9,833 & 44,061 & 52,894 & 1,738 \\
\hline 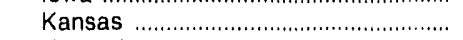 & 544 & 1,077 & 1,620 & 750 \\
\hline 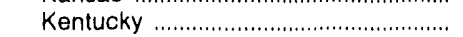 & 3,041 & 34,655 & 37,696 & 24,487 \\
\hline 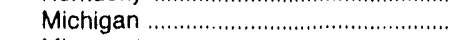 & 38,932 & 202,459 & 241,391 & 16,45 \\
\hline 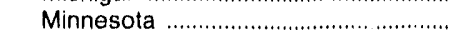 & 48,289 & 179,108 & 227,397 & 11,324 \\
\hline 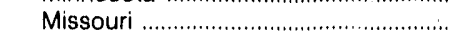 & 5,272 & 18,695 & 23,967 & 2,881 \\
\hline 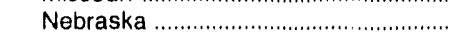 & 6,321 & 6,277 & 12,598 & 344 \\
\hline North Dakota .......................................... & 8,556 & 37,636 & 46,192 & 415 \\
\hline 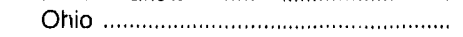 & 24,282 & 207,084 & 231,366 & 36,693 \\
\hline 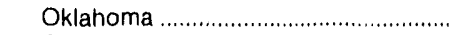 & 1 & 20 & 20 & 812 \\
\hline Suuth Dakota ........................................ & 13.141 & 32,405 & 45,547 & 359 \\
\hline 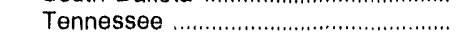 & 161 & 12,213 & 12,373 & 25,858 \\
\hline 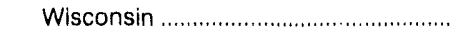 & 31,831 & 241,140 & $2.72,972$ & 1,665 \\
\hline 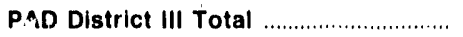 & 101 & 2,130 & 2,231 & $9 ; 422$ \\
\hline 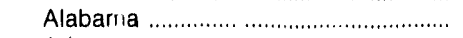 & 14 & 1,041 & 1,055 & 3,412 \\
\hline Arkansas & - & 23 & 23 & 1,214 \\
\hline 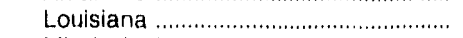 & 19 & 218 & 237 & 1,355 \\
\hline 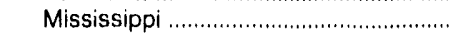 & 41 & 159 & 200 & 950 \\
\hline 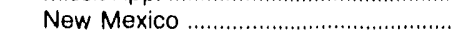 & 21 & 302 & 323 & 424 \\
\hline 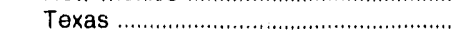 & 7 & 387 & 394 & 2,067 \\
\hline 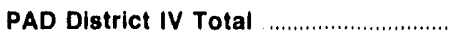 & 12,565 & 35,826 & 48,390 & 2,154 \\
\hline Colorado & 723 & 986 & 1,709 & 1,743 \\
\hline 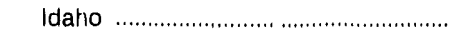 & 4,278 & 18,565 & 22,843 & 99 \\
\hline 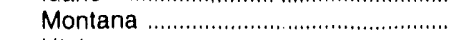 & 6,907 & 8,083 & 14,991 & 42 \\
\hline 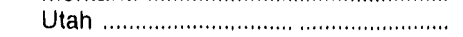 & 361 & 7,126 & 7,488 & 223 \\
\hline 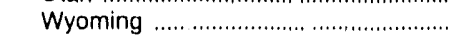 & 296 & 1,065 & 1,361 & 47 \\
\hline PAD District $\vee$ Total $\ldots \ldots \ldots \ldots \ldots \ldots \ldots \ldots$ & 38,268 & 253,981 & 292,250 & 9,088 \\
\hline 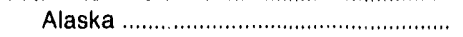 & 20,215 & 46,490 & 66,705 & 31 \\
\hline Arizona & 129 & 167 & 296 & 18 \\
\hline 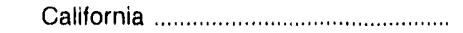 & 3,125 & 7,452 & 10,578 & 4,538 \\
\hline 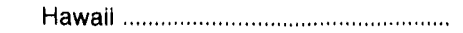 & 4 & 14 & 17 & 0 \\
\hline Nevada & 1,275 & 9,803 & 11,078 & 605 \\
\hline Oregon & 6,079 & 76,909 & 82,988 & 1,608 \\
\hline Washington & 7,441 & 113,146 & 120,587 & 2,288 \\
\hline
\end{tabular}

Dashes (-) :-: No data reported.

Note: Totals may not equal sum of components due to independent rounding.

Sources: - Energy Information Administration Form ElA-821. "Annual Fuel Oil and Kerosene Sales Report," for 1989.

- Kerosene data are based on data from the Energy Information Administration's "Petroleum Supply Annual," Volume 1, 1989, (DOE/EIA. $0340(89) / 1)$ 
Table 8. Sales for Commercial Use: Distillate Fuel Oil, Residual Fuel Oil, and Kerosene, 1989

(Thousand Gallons)

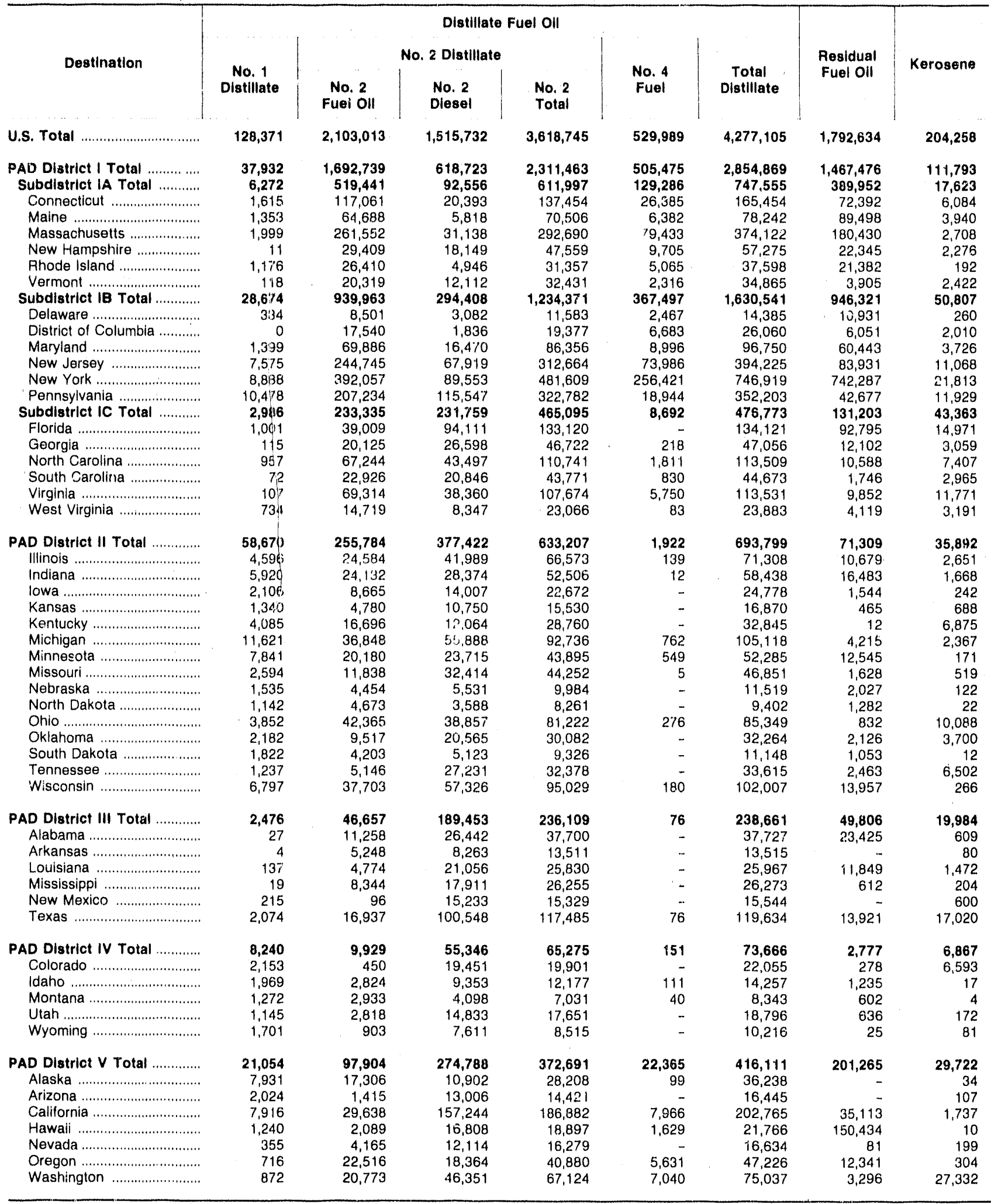

Dashes $(-)=$ No data reported.

Note: Totals may not equal sum of components due to independent rounding.

Sources: - Energy Information Administration Form ElA-821, "Annual Fuel Oil and Kerosene Sales Report," for 1989.

- Kerosene data are based on data from the Energy Information Administration's "Petroleum Supply Annual," Volume 1, 1989, (DOE/EIA$0340(89) / 1)$. 
Table 9. Saies for Industrial Use and for Oil Company Use: Distillate Fuel Oil, Residual Fuel Oil, and Kerosene, 1989

(Thousand Gallons)

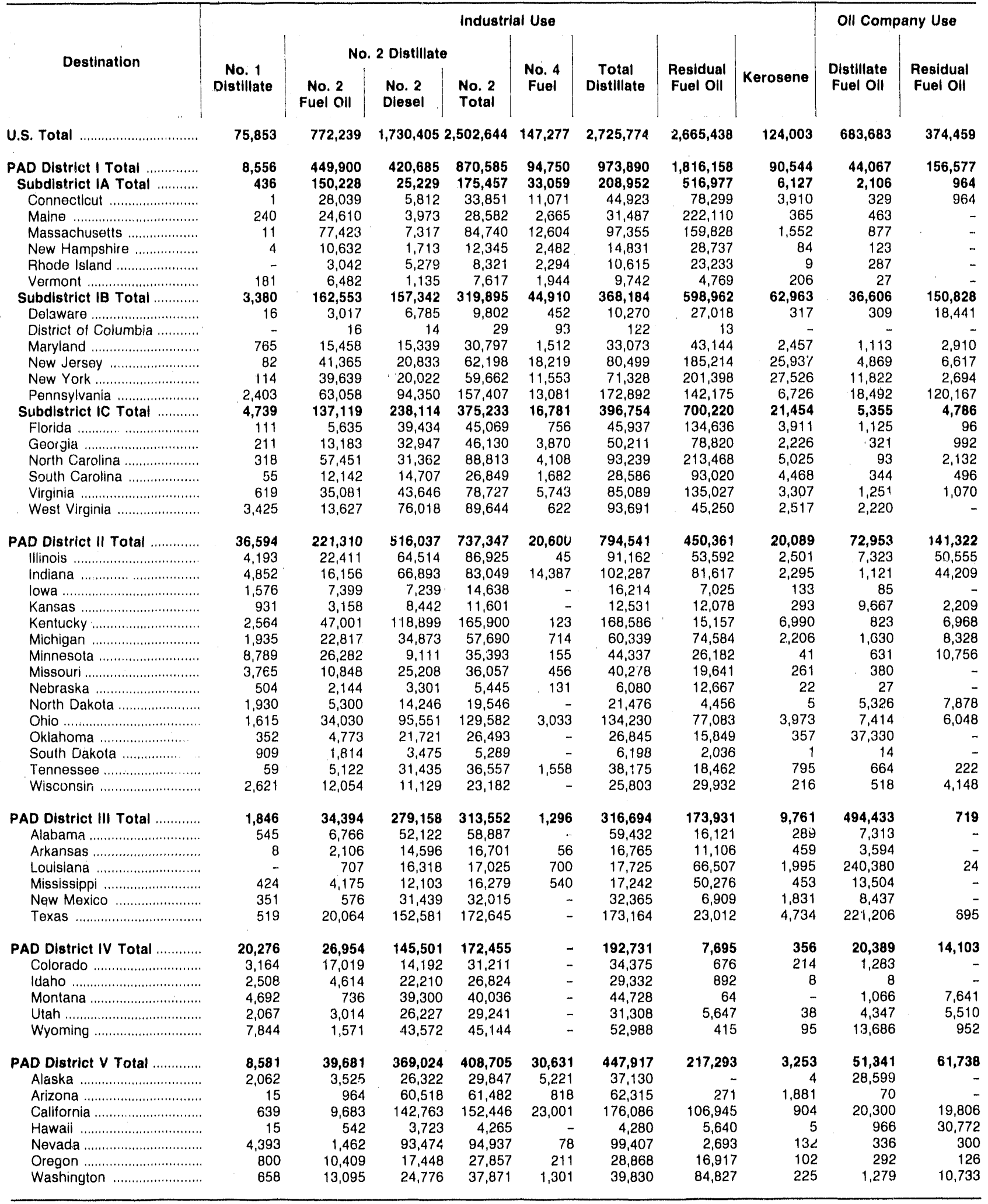

Dashes $(-):=$ No data reported.

Note: Totals may not equal sum of components due to independent rounding.

Sources: - Energy Information Administration Form ElA-821, "Annual Fuel Oll and Kerosene Sales Report," lor 1989.

- Kerosene data are based on data from the Energy information Administration's "Petroleum Supply Annual," Volume 1, 1989, (DOE/EIA $0340(89) / 1)$. 
Table 10. Sales for Farm Use: Distillate Fuel Oil and Kerosene; Sales for Electric Utility Use: Distillate Fuel Oil and Residual Fuel Oil, 1989

(Thousand Gallons)

\begin{tabular}{|c|c|c|c|c|c|c|}
\hline \multirow{2}{*}{ Destination } & \multicolumn{4}{|c|}{ Farm } & \multicolumn{2}{|c|}{ Electric } \\
\hline & Diesel & $\begin{array}{l}\text { Other } \\
\text { Distillate }\end{array}$ & $\begin{array}{c}\text { Total } \\
\text { Distillate }\end{array}$ & Kerosene & $\begin{array}{l}\text { Distillate } \\
\text { Fuel Oll }\end{array}$ & $\begin{array}{l}\text { Residual } \\
\text { Fuel Oil }\end{array}$ \\
\hline 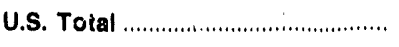 & $3,185,827$ & 174,266 & $3,360,092$ & 30,081 & 887,599 & $9,680,974$ \\
\hline $\begin{array}{l}\text { PAD District I Total ............................ } \\
\text { Subdistrict IA Total ...................... }\end{array}$ & $\begin{array}{r}389,762 \\
12,618\end{array}$ & $\begin{array}{r}67,875 \\
9,203\end{array}$ & $\begin{array}{r}457,637 \\
21,820\end{array}$ & $\begin{array}{r}16,361 \\
2,146\end{array}$ & $\begin{array}{r}516,159 \\
32,525\end{array}$ & $8,498,763$ \\
\hline 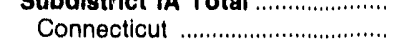 & $\begin{array}{r}12,618 \\
1,722\end{array}$ & $\begin{array}{l}9,203 \\
1,175\end{array}$ & $\begin{array}{r}21,820 \\
2,897\end{array}$ & $\begin{array}{r}2,146 \\
10\end{array}$ & $\begin{array}{r}32,525 \\
4,431\end{array}$ & $\begin{array}{r}2,282,698 \\
740,308\end{array}$ \\
\hline Maine & 3,822 & 1,397 & 5,219 & 1,144 & 2,398 & 217,011 \\
\hline 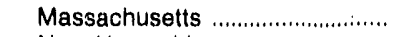 & 1,164 & 2,182 & 3,345 & 89 & 20,113 & $1,089,908$ \\
\hline New Hampshire ........................... & 853 & 1,401 & 2,254 & 54 & 2,720 & 213,234 \\
\hline 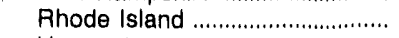 & 113 & 116 & 229 & 1 & 1,613 & 22,237 \\
\hline 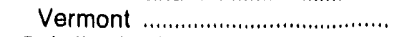 & 4,944 & 2,931 & 7,876 & 848 & 1,248 & - \\
\hline Subdlstrict IB Total ......................... & 112,012 & 53,848 & 165,860 & 8,711 & 330,245 & $4,279,018$ \\
\hline Delaware & 5,580 & 790 & 6,369 & 16 & 11.774 & 171,304 \\
\hline District of Columbia ...................... & - & - & - & - & 59,210 & - \\
\hline 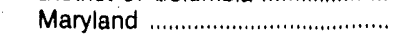 & 23,924 & 3,573 & 27,497 & 1,743 & 52,289 & 399,819 \\
\hline New Jersey ................................... & 14,039 & 7,933 & 21,972 & 7 & 45,090 & 519,702 \\
\hline 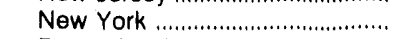 & 33,923 & 22,256 & 56,179 & 4,791 & 93,882 & $2,751,059$ \\
\hline Pennsylvania ................................ & 34,547 & 19,296 & 53,843 & 2,153 & 67,999 & 437,134 \\
\hline Subdistrict IC Total ...................... & 265,132 & 4,824 & 269,956 & 5,504 & 153,389 & $1,937,047$ \\
\hline 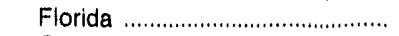 & 54,597 & 45 & 54,642 & 73 & 65,510 & $1,706,257$ \\
\hline 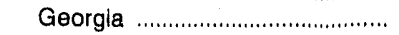 & 95,368 & 6 & 95,374 & 55 & 13,291 & 610 \\
\hline North Carolina .............................. & 43,516 & 1,989 & 45,504 & 1,787 & 18,444 & - \\
\hline South Carolina ............................... & 30,999 & 492 & 31,491 & 1,238 & 9,773 & - \\
\hline 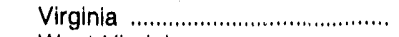 & 35,788 & 1,456 & 37,243 & 1,500 & 27,883 & 230,180 \\
\hline 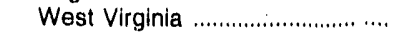 & 4,864 & 837 & 5,702 & 852 & 18,488 & - \\
\hline PAD District II Total ......................... & $1,586,957$ & 91,887 & $1,678,844$ & 10,724 & 154,963 & 195,060 \\
\hline 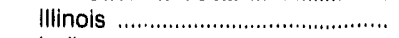 & 191,670 & 13,848 & 205,518 & 536 & 20,280 & 70,987 \\
\hline Indiana & 91,895 & 9,537 & 101,433 & 830 & 23,953 & 0 \\
\hline 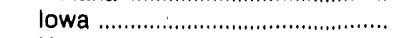 & 168,551 & 9,263 & 177,815 & 552 & 3,975 & - \\
\hline 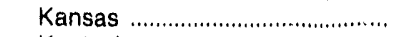 & 156,246 & 3,280 & 159,526 & 490 & 6,144 & - \\
\hline 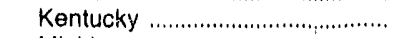 & 44,220 & 2,289 & 46,509 & 871 & 14,074 & - \\
\hline 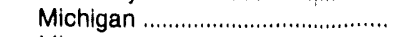 & 68,652 & 3,434 & 72,086 & 295 & 31,072 & 86,356 \\
\hline 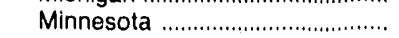 & 149,400 & 6,534 & 155,934 & 1,998 & 5,252 & 6 \\
\hline 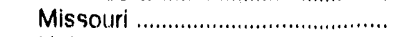 & 93,926 & 1,646 & 95,572 & 381 & 7,915 & 14,287 \\
\hline 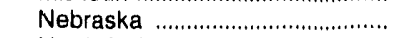 & 172,902 & 2,567 & 175,469 & 417 & 3,211 & 2,501 \\
\hline North Dakota ............................... & 102,035 & 6,271 & 108,306 & 15 & 3,540 & 240 \\
\hline 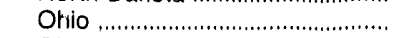 & 81,793 & 8,351 & 90,144 & 3,235 & 17,498 & 20,683 \\
\hline 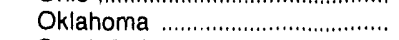 & 49,546 & - & 49,546 & 93 & 446 & - \\
\hline 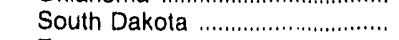 & 80,500 & 9,256 & 89,756 & 198 & 709 & - \\
\hline 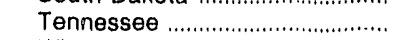 & 47,682 & 26 & 47,708 & 404 & 2,835 & -. \\
\hline 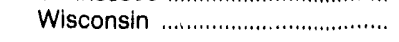 & 87,938 & 15.585 & 103,523 & 407 & 14,080 & - \\
\hline PAD District III Total ......................... & 532,657 & 8,696 & 541,353 & 1,552 & 94,515 & 74,519 \\
\hline 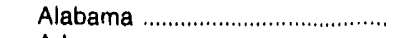 & 43,235 & 892 & 44,127 & 27 & 8,627 & - \\
\hline Arkansas & 67,178 & 201 & 67,379 & 135 & 9,110 & 5,657 \\
\hline 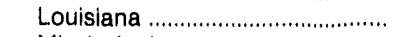 & 66,962 & 159 & 67,122 & 352 & 16,159 & 8,422 \\
\hline Mississippi .................................. & 97,462 & 7 & 97,469 & 439 & 4,173 & 50,793 \\
\hline 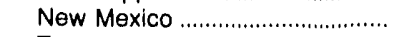 & 12,381 & - & 12,381 & 41 & 5,225 & 1,754 \\
\hline 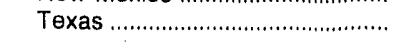 & 245,439 & 7,436 & 252,875 & 558 & 50,623 & 7,893 \\
\hline PAD District IV Total ........................ & 188,165 & 3,059 & 191,225 & 781 & 15,576 & 39 \\
\hline 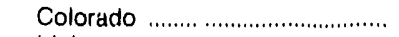 & 52,630 & 655 & 53,285 & 622 & 4,246 & 3 \\
\hline 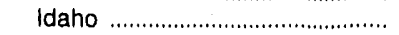 & 66,216 & 336 & 66,552 & 22 & 22 & - \\
\hline 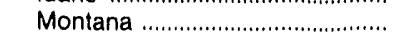 & 51,229 & 1,277 & 52,506 & 91 & 2,100 & - \\
\hline 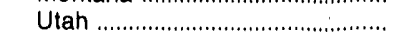 & 9,108 & 305 & 9,413 & 23 & 3,532 & - \\
\hline 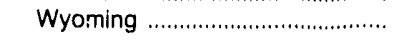 & 8,982 & 486 & 9,469 & 22 & 5,676 & 36 \\
\hline PAD District $\vee$ Total ......................... & 488,286 & 2,749 & 491,034 & 664 & 106,386 & 912,593 \\
\hline 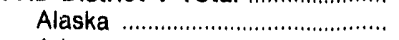 & 269 & - & 269 & - & 18,061 & - \\
\hline Arizona & 33,116 & - & 33,116 & 17 & 3,605 & 3,352 \\
\hline 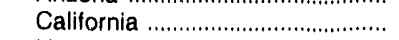 & 316,145 & 10 & 316,155 & 501 & 16,223 & 368,484 \\
\hline 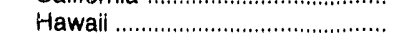 & 10,478 & 1,171 & 11,649 & 0 & 61,077 & 516,208 \\
\hline 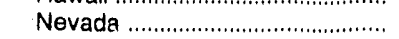 & 5,283 & 146 & 5,428 & 43 & 2,171 & 24,548 \\
\hline Oregon & 51,458 & 73 & 51,532 & 53 & 4,412 & $2-$ \\
\hline 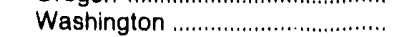 & 71,537 & 1,348 & 72,885 & 50 & 837 & - \\
\hline
\end{tabular}

Dashes $(-)=$ No data reported.

Note: Totals may not equal sum of components due to independent rounding.

Sources: - Energy Information Administration Form ElA-821. "Annual Fuel Oil and Kerosene Sales Feport," for 1989

- Kerosene data are based on data from the Energy Information Administration's "Petroleum Supply Annual," Volume 1, 1989, (DOE/EIA. $0340(89) / 1)$ 
Table 11. Sales for Transportation Use: Distillate Fuel Oil and Residual Fuel Oil, 1989

(Thousand Gallons)

\begin{tabular}{|c|c|c|c|c|c|c|c|}
\hline \multirow[b]{2}{*}{ Destination } & \multicolumn{2}{|c|}{ Rallroad Use } & \multicolumn{2}{|c|}{ Vessel Bunkering } & \multirow{2}{*}{$\begin{array}{c}\text { On-Highway } \\
\text { Diesel } \\
\text { Distlllate } \\
\text { Fuel Oll }\end{array}$} & \multicolumn{2}{|c|}{ Total Transportation Use } \\
\hline & $\begin{array}{l}\text { Dissillate } \\
\text { Fuel Oll }\end{array}$ & $\begin{array}{l}\text { Residual } \\
\text { Fuel Oll }\end{array}$ & $\begin{array}{l}\text { Distillate } \\
\text { Fuel Oll }\end{array}$ & $\begin{array}{l}\text { Residual } \\
\text { Fuel Oll }\end{array}$ & & $\begin{array}{l}\text { Disitillate } \\
\text { Fubl Oil }\end{array}$ & $\begin{array}{l}\text { Residual } \\
\text { Fuel Oll }\end{array}$ \\
\hline U.S. Total & $3,240,649$ & 39 & $2,258,465$ & $7,191,689$ & $21,131,692$ & $26,6: 30,806$ & $7,191,727$ \\
\hline 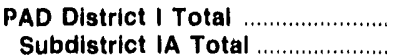 & $\begin{array}{r}551,268 \\
35,062\end{array}$ & - & $\begin{array}{r}521,110 \\
67,894\end{array}$ & $\begin{array}{r}1,381,741 \\
49,484\end{array}$ & $\begin{array}{r}6,752,738 \\
827956\end{array}$ & $7,825,116$ & $1,381,741$ \\
\hline 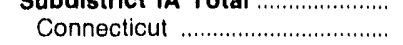 & $\begin{array}{r}30,002 \\
5,374\end{array}$ & - & $\begin{array}{r}01,694 \\
2,249\end{array}$ & $\begin{array}{r}49,404 \\
341\end{array}$ & $\begin{array}{l}827,956 \\
257,361\end{array}$ & $\begin{array}{l}930,912 \\
264,983\end{array}$ & $\begin{array}{r}49,484 \\
341\end{array}$ \\
\hline 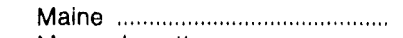 & 6,377 & - & 14,097 & 5,729 & 139,908 & 60,382 & 5,729 \\
\hline 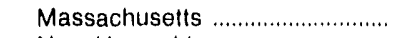 & 19,591 & - & 43,961 & 42,742 & 271,944 & 335,496 & 42,742 \\
\hline New Hampshire .......................... & 36 & - & 387 & - & 59,561 & 59,984 & - \\
\hline 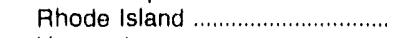 & 530 & - & 6,778 & 671 & 52,932 & 60,240 & 671 \\
\hline 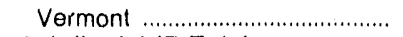 & 3,154 & - & 422 & - & 46,250 & 49,826 & - \\
\hline 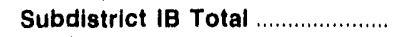 & 178,330 & - & 191,904 & 758,807 & $2,571,926$ & $2,942,160$ & 758,807 \\
\hline 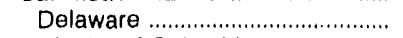 & 1,030 & - & 3,746 & 41,561 & 74,089 & 78,866 & 41,561 \\
\hline District of Columbia ..................... & 13,191 & - & 25 & - & 23,064 & 36,280 & - \\
\hline 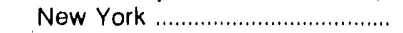 & 17,457 & - & 11,409 & 17,095 & 754,276 & 783,142 & 17,095 \\
\hline Pennsylvania .................................... & 43,679 & - & 29,720 & 189,574 & 915,865 & 989,265 & 189,574 \\
\hline Subdistrict IC Total & 337,876 & - & 261,312 & 573,450 & $3,352,856$ & $3,952,044$ & 573,450 \\
\hline 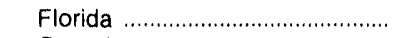 & 84,939 & - & 103,032 & 372,112 & 899,369 & $1,087,34 t$ & 372,112 \\
\hline 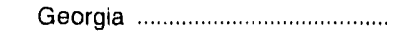 & 65,462 & - & 40,099 & 29,423 & 838,847 & 944,408 & 29,423 \\
\hline 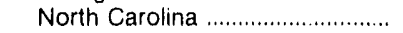 & 36,898 & - & 9,930 & 27,619 & 614,485 & 661,314 & 27,619 \\
\hline 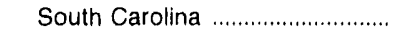 & 30,723 & - & 6,186 & 25,874 & 302,614 & 339,523 & 25,874 \\
\hline Virginia & 93,726 & - & 29,071 & 118,421 & 537,569 & 660,366 & $118,42.1$ \\
\hline 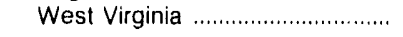 & 26,127 & - & 72,993 & - & 159,972 & 259,093 & - \\
\hline PAD District \| Total ......... & $1,326,339$ & 1 & 458,790 & 18,710 & $7,253,324$ & $9,038,453$ & 18,710 \\
\hline 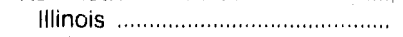 & 138,438 & - & 39,678 & 2,671 & 839,968 & $1,018,084$ & 2,671 \\
\hline 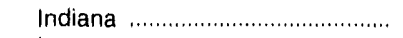 & 225,525 & - & 11,125 & 9,908 & 784,441 & $1,021,091$ & 9,908 \\
\hline 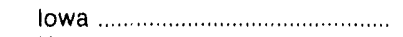 & 33,695 & 1 & 2,896 & - & 349,044 & 385,635 & 1 \\
\hline 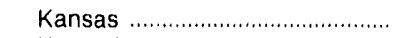 & 168,700 & - & - & - & 303,893 & 472,593 & - \\
\hline 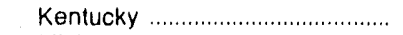 & 115,661 & - & 118,211 & - & 692,215 & 926,087 & - \\
\hline 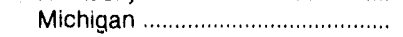 & 42,376 & - & 5,491 & 5,397 & 528,906 & 576,773 & 5,397 \\
\hline 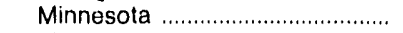 & 39,159 & - & 3,221 & 73 & 331,625 & 374,005 & 73 \\
\hline 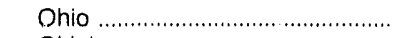 & 122,714 & - & 10,171 & 451 & 960,195 & $1,093,081$ & 451 \\
\hline 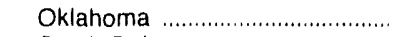 & 77,737 & - & 321 & - & 394,174 & 472,232 & - \\
\hline 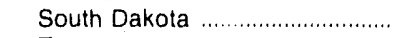 & 2,357 & - & - & - & 91,221 & 93,578 & - \\
\hline 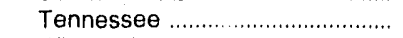 & 61,730 & - & 210,942 & 22 & 587,396 & 860,068 & 22 \\
\hline 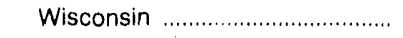 & 105,521 & - & 3,641 & 187 & 449,764 & 558,926 & 187 \\
\hline PAD District III Total .......................... & 636,216 & - & 730,531 & $2,535,626$ & $3,221,237$ & $4,587,983$ & $2,535,626$ \\
\hline 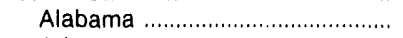 & 66,745 & - & 102,207 & 130,922 & 544,882 & 713,835 & 130,922 \\
\hline 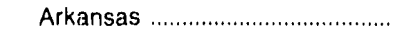 & 34,945 & - & 487 & - & 395,236 & 430,669 & - \\
\hline 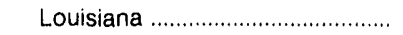 & 78,426 & - & 336,513 & $1,130,135$ & 385,621 & 800,560 & $1,130,135$ \\
\hline 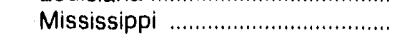 & 17,760 & - & 55,534 & 56,303 & 302,523 & 375,818 & 56,303 \\
\hline 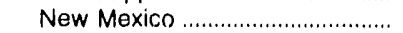 & 51,784 & - & - & - & 205,916 & 257,700 & - \\
\hline 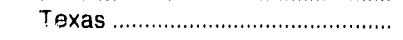 & 386,555 & - & 235,789 & $1,218,266$ & $1,387,059$ & $2,009,402$ & $1,218,266$ \\
\hline 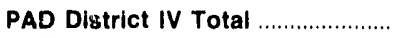 & 310,192 & 19 & 131 & - & 734,904 & $1,045,226$ & 19 \\
\hline 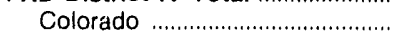 & 87,970 & - & 62 & - & 185,519 & 273,551 & - \\
\hline 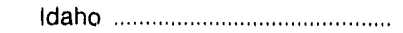 & 19,585 & - & 69 & - & 117,246 & 136,900 & - \\
\hline 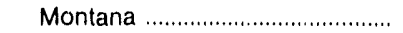 & 58,855 & - & - & - & 119,503 & 178,358 & .. \\
\hline 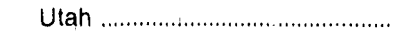 & 34,590 & 9 & - & - & 125,184 & 159,774 & 9 \\
\hline 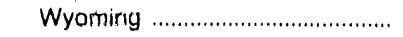 & 109,191 & 11 & - & - & 187,452 & 296,643 & 11 \\
\hline PAD District V Total ........................ & 416,635 & 19 & 547,903 & $3,255,612$ & $3,169,489$ & $4,134,027$ & $3,255,631$ \\
\hline 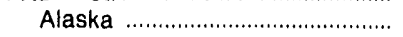 & 4,927 & - & 138,221 & 5,530 & 96,446 & 239,595 & 5,530 \\
\hline Arizona & 8,288 & - & - & - & 314,671 & 322,959 & - \\
\hline 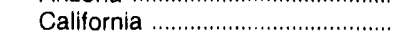 & 216,816 & - & 208,063 & $2,297,366$ & $1,913,647$ & $2,338,526$ & $2,297,366$ \\
\hline 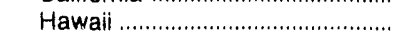 & 17 & - & 86,679 & 101,297 & 23,717 & 110,413 & 101,297 \\
\hline 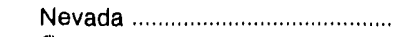 & 23,341 & - & - & - & 131,055 & 154,396 & - \\
\hline 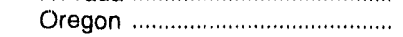 & 85,764 & 19 & 35,865 & 222,279 & 311,843 & 433,473 & 222,298 \\
\hline 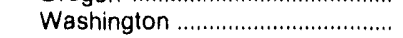 & 77,481 & - & 79,074 & 629,140 & 378,110 & 534,665 & 629,140 \\
\hline
\end{tabular}

Dashes $(\cdot)=$ No data reported.

Note: Totals may not equal sum of components due to independent rounding.

Sources: - Energy Information Administration Form ElA-821, "Annual Fuel Oll and Kerosene Sales Report," for 1989.

- On-Highway Diesel data are Federal Highway Administation statistics of highway special fuels use. 
Tabie 12. Sales for Military, Off-Highway, and All Other Uses: Distillate Fuel Oil, Residual Fuel Oil, and Kerosene, 1989 (Thousand Gallons)

\begin{tabular}{|c|c|c|c|c|c|c|c|c|c|c|}
\hline \multirow{2}{*}{ Destination } & \multicolumn{3}{|c|}{ Military } & \multicolumn{4}{|c|}{$\begin{array}{l}\text { Off-Highway Diesel } \\
\text { Distillate Fuel Oll }\end{array}$} & \multicolumn{3}{|c|}{ All Other } \\
\hline & Diesel & $\begin{array}{l}\text { Other } \\
\text { Distillate }\end{array}$ & $\begin{array}{c}\text { Total } \\
\text { Distillate }\end{array}$ & $\begin{array}{l}\text { Residual } \\
\text { Fuel OII }\end{array}$ & Construction & Other & Total & $\begin{array}{l}\text { Distillate } \\
\text { Fuel Oll }\end{array}$ & $\begin{array}{l}\text { Residual } \\
\text { Fuel Oil }\end{array}$ & Kerosene \\
\hline U.S. Total & 774,168 & 78,671 & 852,839 & 92,229 & $1,504,932$ & 184,719 & $1,689,651$ & 32,719 & 31,838 & 64,598 \\
\hline $\begin{array}{l}\text { PAD District I Total .............. } \\
\text { Subdistrict IA Total .............. }\end{array}$ & $\begin{array}{l}97,901 \\
12,552\end{array}$ & $\begin{array}{r}64,609 \\
4,809\end{array}$ & $\begin{array}{r}162,510 \\
17,361\end{array}$ & $\begin{array}{r}79,609 \\
22,627\end{array}$ & $\begin{array}{r}488,476 \\
69,375\end{array}$ & $\begin{array}{r}42,203 \\
7,073\end{array}$ & $\begin{array}{r}530,679 \\
76,448\end{array}$ & $\begin{array}{r}12,998 \\
687\end{array}$ & $\begin{array}{r}208 \\
-\end{array}$ & $\begin{array}{r}49,553 \\
8,001\end{array}$ \\
\hline 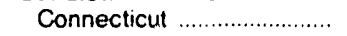 & 73 & 629 & 702 & 2,714 & 21.475 & 37 & 21,512 & 551 & - & 6,137 \\
\hline Maine ........... & 3,130 & 300 & 3,431 & 3.580 & 10,398 & 4,357 & 14,755 & 45 & - & $53 C$ \\
\hline Massachusetts & 1,456 & 829 & 2,285 & 12,607 & 20,927 & 17 & 20,943 & - & - & 768 \\
\hline New Hampshire ................... & 350 & 1.422 & 1,772 & 919 & 7.399 & 1,422 & 8,821 & - & - & 457 \\
\hline Rhode Island .......................... & 7,461 & 1,446 & 8,907 & 2,497 & 3,842 & 1 & 3,844 & 91 & - & 36 \\
\hline 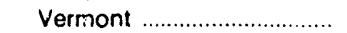 & 81 & 183 & 264 & 310 & 5,334 & 1,238 & 6,572 & - & - & 72 \\
\hline 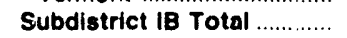 & 31,463 & 32,487 & 63,949 & 35,854 & 197,196 & 10,869 & 208,065 & 3,838 & - & 36,555 \\
\hline Delaware & - & 98 & 98 & - & 4,827 & 3 & 4,830 & - & - & 35 \\
\hline District of Columbia ............ & 8 & 3,436 & 3,444 & 1,853 & 36 & - & 36 & - & - & - \\
\hline 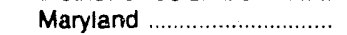 & 16,997 & 8,679 & 25,677 & 20,756 & 35,675 & 1,300 & 36.975 & 2,954 & - & 189 \\
\hline 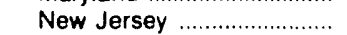 & 4,742 & 5,747 & 10,489 & 2,534 & 58,193 & 2,104 & 60,297 & 55 & - & 3,584 \\
\hline New York ..... & 6,820 & 5.147 & 11,967 & 7,724 & 39,786 & 6,539 & 46,325 & 4 & - & 30,988 \\
\hline Pennsylvania & 2,895 & 9,379 & 12,274 & 2,986 & 58,679 & 922 & 59,601 & 825 & - & 1,758 \\
\hline Subdiatrict IC Total ............ & 53,887 & 27,314 & 81,200 & 21,128 & 221,904 & 24,262 & 246,166 & 8,474 & 208 & 4,998 \\
\hline 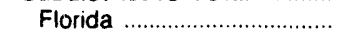 & 23,327 & 15 & 23,342 & 6,537 & 71,415 & 4,027 & $75,4.42$ & 1,503 & - & 842 \\
\hline 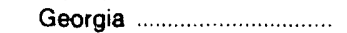 & y,981 & 541 & 10,522 & 1,706 & 40,588 & 3,301 & 43,889 & 1,851 & - & 409 \\
\hline North Carolina ..................... & -814 & 1,146 & 8,960 & 3,693 & 29,034 & 1,607 & 30,641 & 2,568 & 208 & 898 \\
\hline 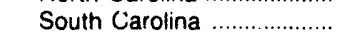 & 2,389 & 550 & 2,939 & 5,558 & 18,120 & 12,460 & 30,580 & 1,044 & - & 1,428 \\
\hline Virginia & 10,248 & 25.003 & 35,251 & 3,635 & 40,853 & 1,359 & 42,212 & 1,493 & - & 1,077 \\
\hline West Virginia .......................... & 128 & 59 & 187 & - & 21,894 & 1,508 & 23,402 & 15 & - & 345 \\
\hline PAD District II Total. & 46,933 & 6,767 & 53,700 & 3,760 & 387,145 & 22,921 & 410,067 & 16,777 & 464 & 5,842 \\
\hline Illinois & 4,666 & 671 & 5.337 & - & 44,767 & 478 & 45,245 & 194 & - & 999 \\
\hline Indiana & 7,760 & 154 & 7,914 & - & 33,763 & 1,738 & 35,500 & 3,397 & - & 169 \\
\hline lowa & 296 & 31 & 327 & 8 & 10,364 & 1,197 & 11,561 & 2,260 & - & 313 \\
\hline 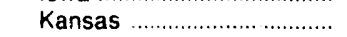 & 905 & 125 & 1,030 & - & 13,091 & 1,791 & 14,882 & 1,928 & - & 124 \\
\hline 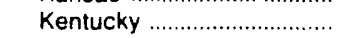 & 5,482 & 1,047 & 6,529 & - & 25,486 & 793 & 26,279 & 23 & .- & 388 \\
\hline 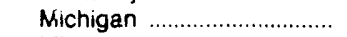 & 3,176 & 615 & 3,791 & - & 52,963 & 1,732 & 54,695 & 2,101 & 62 & 379 \\
\hline Minnesota & 2,190 & 255 & 2,445 & - & 28,472 & 3,050 & 31,523 & 2,190 & 130 & 58 \\
\hline Missouri .............. & 3,860 & 2,157 & 6,017 & 3,250 & 24,671 & 1,231 & 25,902 & 1,403 & - & 742 \\
\hline Nebraska ........ & 1,190 & 91 & 1,281 & - & 20,283 & 299 & 20,582 & - & 16 & 11 \\
\hline North Dakota ... & 1,603 & 12 & 1,620 & - & 4,749 & 683 & 5,433 & 224 & 256 & 11 \\
\hline 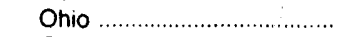 & 10,159 & 199 & 10,358 & - & 32,705 & 986 & 33,691 & 404 & - & 2,151 \\
\hline 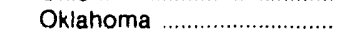 & 1,324 & 472 & 1,796 & - & 24,883 & 1,680 & 26,563 & 274 & - & 60 \\
\hline South Dakota ......................... & 585 & 31 & 616 & 2 & 6,678 & 120 & 6,798 & 651 & - & 33 \\
\hline 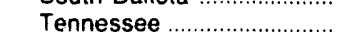 & 2,772 & 583 & 3,355 & 500 & 29,644 & 2,428 & 32,072 & 783 & .. & 330 \\
\hline 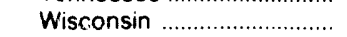 & 958 & 324 & 1,282 & - & 34,626 & 4,715 & 39,341 & 935 & - & 73 \\
\hline PAD District III Total & 323,328 & 74 & 323,472 & 1,077 & 247,229 & 28,540 & 275,769 & 2,505 & - & 8,233 \\
\hline Alabama & 4,561 & 73 & 4,634 & 1,077 & 38,914 & 7,810 & 46,724 & 1,463 & - & 412 \\
\hline 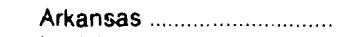 & 1,514 & - & 1,514 & - & 16,719 & 6.784 & 23,503 & 46 & - & 66 \\
\hline Louisiana. & 60,773 & - & 60,773 & - & 39,230 & 986 & 40,216 & 201 & - & 1,884 \\
\hline Mississippi & 53,722 & 1 & 53,723 & - & 20,295 & 4,267 & 24,562 & 118 & - & 673 \\
\hline New Mexico. & 667 & - & 667 & - & 15,053 & 663 & 15,716 & 35 & - & 64 \\
\hline Texas & 202,160 & - & 202,160 & - & 117,017 & 8,029 & 125,047 & 643 & - & 5.135 \\
\hline PAD District IV Total ............ & 11,215 & 2,167 & 13,402 & - & 80,700 & 10,561 & 91,261 & 438 & 225 & 360 \\
\hline Colorado & 8,144 & - & 8,144 & - & 26,225 & 443 & 26.668 & 45 & - & 329 \\
\hline 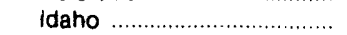 & 720 & - & 720 & - & 10,732 & 2,792 & 13,524 & 25 & - & 5 \\
\hline 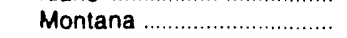 & 213 & 129 & 342 & - & 8,755 & 6,832 & 15,587 & 24 & 197 & 4 \\
\hline Utah .......... & 1,958 & 2,058 & 4,016 & - & 17,757 & 329 & 18,086 & - & - & 10 \\
\hline Wyoming . & 181 & - & 181 & - & 17,231 & 165 & 17,396 & 344 & 28 & 12 \\
\hline PAD District $\vee$ Total ............... & 294,721 & 5,033 & 299,754 & 7,783 & 301,382 & 80,493 & 381,876 & - & 30,942 & 610 \\
\hline Alaska & 35,535 & 122 & 35,657 & - & 8,983 & 5.643 & 14,630 & - & - & 12 \\
\hline Arizona .............. & 2,350 & 8 & 2,358 & - & 37.165 & 2.576 & 39,741 & - & - & 63 \\
\hline 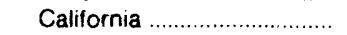 & 215,013 & - & 215,013 & - & 175,637 & 26,599 & 202,235 & - & - & 392 \\
\hline Hawail ........... & 32,595 & - & 22,595 & 3.196 & 5,621 & 59 & 5,680 & - & 30,877 & - \\
\hline Nevada .............. & 3,781 & 824 & 4,605 & - & 24,910 & 273 & 25,183 & - & - & 116 \\
\hline Oregon & 714 & 53 & 767 & - & 16,529 & 30,119 & 46,647 & - & 65 & 17 \\
\hline 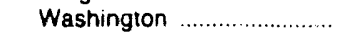 & 4,733 & 4,026 & 8,758 & 4,587 & 32,534 & $i 5,225$ & 47,759 & - & - & 9 \\
\hline
\end{tabular}

Dashes $(\cdot)=$ No data reported.

Note: Totals may not equal sum of components due to independent rounding

Sources: - Energy Information Administration Form ElA-821, "Annual Fuel Oil and Kerosene Sales Report," for 1989. • Kerosenn data are based on data from the Energy Information Administration's "Petroleum Supply Annual," Volume 1, 1989, (DOE/EIA.
$0340(89) / 1$ ). 


\section{Adjusted Sales of Fuel Oil and Kerosene in 1989}

The following tables contain estimates which have been adjusted to ensure that PAD vistricts total sales of distillate and residual fuel oils and kerosene equal published EIA volume estimates of products supplied in the U.S. marketplace. The products supplied estimates can be found in the 1985 through 1989 Petroleum Supply Annual. For further discussion of these adjustments, see Technical Note 3 . As noted in the Introduction, Tables 13 through 24 will now be titled "Adjusted Sales of Fuel Oil and Kerosene."
Total domestic adjusted sales of fuel oil and kerosene remained constant from 1988 to $1989,70.6$ billion gallons to 70.7 billion gallons. While adjusted sales of distillate fuel oil showed a 0.9 percent increase over 1988 , adjusted sales of residual fuel oil declined by 0.8 percent in 1989. Adjusted sales of kerosene showed a dramatic decrease, declining 12.6 percent from the previous year. 
Table 13. Adjusted Sales of Distillate Fuel Oil by End Use in the United States: 1985-1989

(Thousand Gallons)

\begin{tabular}{|c|c|c|c|c|c|}
\hline \multirow{3}{*}{ End Use } & \multicolumn{5}{|c|}{ Distillate Fuel OII } \\
\hline & & & & & \\
\hline & 1985 & 1986 & 1987 & 1988 & 1989 \\
\hline 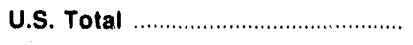 & $43,966,734$ & $44,677,080$ & $45,629,346$ & $47,985,420$ & $48,393,450$ \\
\hline 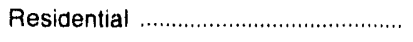 & $7,212,897$ & $7,294,814$ & $7,426,412$ & $7,662,046$ & $7,501,098$ \\
\hline 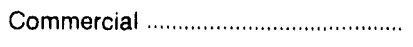 & $4,514,211$ & $4,293,095$ & $4,279,375$ & $4,135,079$ & $3,858,743$ \\
\hline 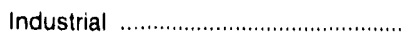 & $2,592,678$ & $2,684,102$ & $2,917,624$ & $2,617,639$ & $2,565,293$ \\
\hline Oil Company & 876,505 & 749,992 & 881,729 & 872,133 & 845,217 \\
\hline Farm & $3,311,309$ & $3,380,046$ & $3,241,968$ & $3,420,671$ & $3,196,928$ \\
\hline 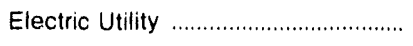 & 514,327 & 614,361 & 646,073 & 800,085 & $1,078,887$ \\
\hline 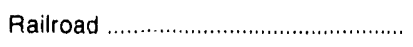 & $3,209,729$ & $3,099,616$ & $3,147,661$ & $3,259,250$ & $3,264,229$ \\
\hline On-Highway Diesel ............................ & $17,279,650$ & $17,926,508$ & $18,172,934$ & $20,038,691$ & $21,131,692$ \\
\hline 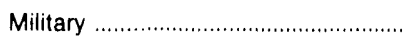 & 768,179 & 760,417 & 884,392 & 980,877 & 928,316 \\
\hline Off-Highway Diesel ............................ & $1,616,685$ & $1,699,252$ & $1,732,106$ & $1,821,819$ & $1,637,497$ \\
\hline 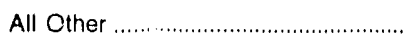 & 176,299 & 140,661 & 75,815 & 66,763 & 29,106 \\
\hline
\end{tabular}

Note: Totals may not equal sum of components due to independent rounding.

Sources: - Energy Information Administration Form ElA.821, "Annual Fuel Oil and Kerosene Sales Report," for 1985.1989.

- Electric Utility data are based on data obtained on Form EIA-759, "Monthiy Power Plant Report," and Form FERC-423, "Monthly Report of Cost and Quality of Fuels for Elestric Plants."

- Railroad data for distillate fuel oil are based on Association of American Railroads' statistics for railroads of Class I and Amtrak Companies.

- On-Highway Diesel data are Federal Highway Administation statistics of highway special fuels use

- Energy Information Administration's "Petroleum Supply Annual," Volume 1, 1985-1989, (DOE/EIA-0340(85)/1 - (89)/1). 


\section{Table 14. Adjusted Sales of Residual Fuel Oil by End Use in the United States: 1985-1989 \\ (Thousand Gallons)}

\begin{tabular}{|c|c|c|c|c|c|}
\hline \multirow{3}{*}{ End Use } & \multicolumn{5}{|c|}{ Residual Fuel DII } \\
\hline & $\cdot \cdot$ & & & & \\
\hline & 1985 & 1986 & 1987 & 1988 & 1989 \\
\hline 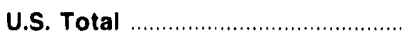 & $18,431,280$ & $21,744,114$ & $19,383,168$ & $21,179,550$ & $21,002,814$ \\
\hline 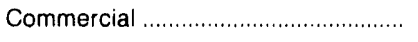 & $1,326,870$ & $1,663,005$ & $1,764,531$ & 1788,728 & $1,537,027$ \\
\hline 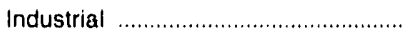 & $4,011,361$ & $4,492,393$ & $3,810,345$ & $3,855,070$ & $3,712,664$ \\
\hline Oil Company ......................................... & 776,019 & 766,034 & 502,615 & 433,294 & 403,191 \\
\hline 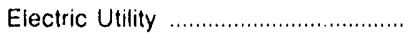 & $6,183,985$ & $9,041,608$ & $7,631,898$ & $9,540,878$ & $9,797,966$ \\
\hline 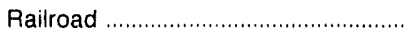 & 6,163 & 569 & 294 & 33 & 151 \\
\hline Vessel Bunkering & $5,473,562$ & $5,287,347$ & $5,259,272$ & $5,248,981$ & $5,410,263$ \\
\hline 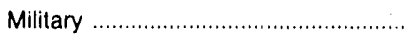 & 192,918 & 180,918 & 191,045 & E 137,287 & 105,255 \\
\hline 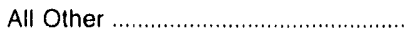 & 460,402 & 312,241 & 223,168 & 175,279 & 36,298 \\
\hline
\end{tabular}

E Estimated data. Annual estimate is based on eleven months of data

Note: Totals may not equal sum of components due to independent rounding

Sources: - Energy iriformation Administration Form ElA-821, "Annual Fuel Oil and Kerosene Sales Report," for 1385-1989.

- Electric Utility data are based on data obtained on Form ElA-759, "Monthly Power Plant Report," and Form FERC-423, "Monthly Report of Cost and Quality of Fuels for Electric Plants."

- Vessel Bunkering data for residual fuel oil are based on ElA estimates of intercoastal bunkering and the Bureau of the Census Report, "United States Foreign Trade, Buriker Fuels."

- Military data for residual fuel oil are based on Defense Fuel Supply Center's "DEIS Report."

- Energy Information Administration's "Petroleum Supply Annual," Volume 1, 1985-1989, (DOE/EIA-0340(85)/1 - (B9)/1).

\section{Table 15. Adjusted Sales of Kerosene by End Use in the United States: $1985-1989$ \\ (Thousand Gallons)}

\begin{tabular}{|c|c|c|c|c|c|}
\hline \multirow{3}{*}{ End Use } & \multicolumn{5}{|c|}{ Kerosene } \\
\hline & & & & & \\
\hline & 1985 & 1986 & 1997 & 1988 & 1989 \\
\hline 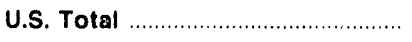 & $1,745,856$ & $1,506,162$ & $1,449,504$ & $1,478,316$ & $1,291,668$ \\
\hline 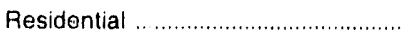 & 862,573 & 641.742 & 671,350 & 770,042 & 618,322 \\
\hline Comınercial ..................................... & 367,218 & 307,704 & 267,437 & 306,584 & 349,566 \\
\hline Industrial ...... & 254,491 & 173,859 & 160,057 & 188,117 & 171,124 \\
\hline 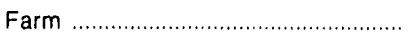 & 53,434 & 47,948 & 34,761 & 45,990 & 36,907 \\
\hline 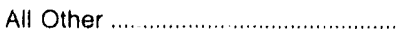 & 208,139 & 334,908 & 315,900 & 167,583 & 115,750 \\
\hline
\end{tabular}

Note: Totals may not equal sum of components due to independent rounding

Note: Kerosene data in the Adjusted Sales tables (13-24) are adjusted at the PAD District level. See Technical Note 3 for further explanation.

Sources: - Kerosene data are based on data from the Energy Information Adininistration's "Petroleum Supply Annual," Volume 1, 1985-1989, (DOE/EIA-0340(85)/1 - (89)/1) 
Table 16. Adjusted Sales of Distillate Fuel Oil by End Use, 1988 and 1989

(Thousand Gallons)

\begin{tabular}{|c|c|c|c|c|c|c|}
\hline \multirow{3}{*}{ Destination } & \multicolumn{2}{|c|}{ Residential } & \multicolumn{2}{|c|}{ Commercial } & \multicolumn{2}{|c|}{ Industrial } \\
\hline & & & & & & \\
\hline & 1988 & 1989 & 1988 & 1989 & 1988 & 1989 \\
\hline 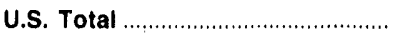 & $7,662,046$ & $7,501,098$ & $4,135,079$ & $3,858,743$ & $2,617,639$ & $2,565,293$ \\
\hline PAD District I Total. & $6,019,068$ & $6,003,771$ & $2,370,013$ & $2,483,447$ & 882,413 & 847,186 \\
\hline 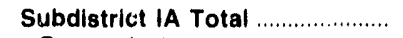 & $1,968,313$ & $2,103,316$ & 580,541 & 650,297 & 179,249 & $\{81,767$ \\
\hline Connecticut ................................... & 551,570 & 597,477 & 126,954 & 143,928 & 41,539 & 39,079 \\
\hline Massachusetts ........................... & 778,311 & 862,166 & 267,161 & 325,448 & 81,932 & 84,689 \\
\hline New Hampshire ........................... & 155,027 & 180,907 & 48,426 & 49,823 & 12,381 & 12,902 \\
\hline 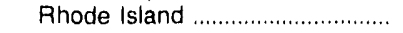 & 151,241 & 133,478 & 33,914 & 32,707 & 9,660 & 9,234 \\
\hline 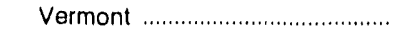 & 81,504 & 90,843 & 29,000 & 30,329 & 8,010 & 8,474 \\
\hline 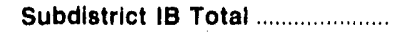 & $3,413,354$ & $3,332,803$ & $1,301,863$ & $1,418,405$ & $369,59.5$ & 320,283 \\
\hline 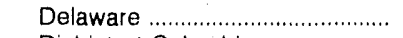 & 58,492 & 55,486 & 16,393 & 12,513 & 8,396 & 8,934 \\
\hline District of Columbia .................... & 12,392 & 6,115 & 22,978 & 22,669 & 41 & 107 \\
\hline 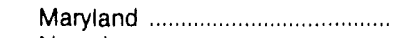 & 248,603 & 215,803 & 78,171 & 84,163 & 28,327 & 28,770 \\
\hline 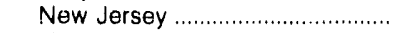 & 733,996 & 668,788 & 331,693 & $\checkmark 42,936$ & 84,121 & 70,026 \\
\hline Subcilstrict IC Total ........................ & 637,401 & 567,651 & 487,609 & 414,744 & 333,569 & 345,136 \\
\hline 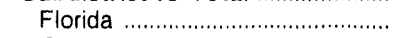 & 21,929 & 15,238 & 139,057 & 116,672 & 34,013 & 39,961 \\
\hline 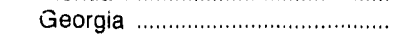 & 10,821 & 11,208 & 50,504 & 40,934 & 36.948 & 43,679 \\
\hline 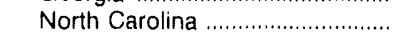 & 240,830 & 196,372 & 129,619 & 98,741 & 81,008 & 81,108 \\
\hline 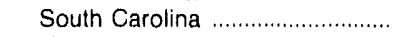 & 47,027 & 53,820 & 44,277 & 38,861 & 21,804 & 24,867 \\
\hline 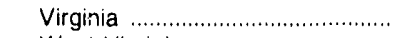 & 292.714 & 263,958 & 109,142 & 98,760 & 77,482 & 74.019 \\
\hline 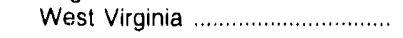 & 24,079 & 27,056 & 15,011 & 20,776 & 82,313 & 81,502 \\
\hline PAD District $\|$ Total ....................... & $1,313,802$ & $1,165,361$ & 717,889 & 575,845 & 718,861 & 659,460 \\
\hline Illinois & 89,088 & 66,377 & 82,151 & 59,185 & 72.447 & 75,664 \\
\hline Indiana & 117,319 & 97,159 & 56,174 & 48,503 & 71,576 & 84.897 \\
\hline 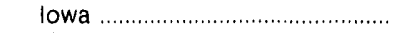 & 46,844 & 44,732 & 28,766 & 20,565 & 19,781 & 13,458 \\
\hline 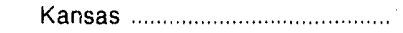 & 1,254 & 1,345 & 16,178 & 14,002 & 14,194 & 10,401 \\
\hline 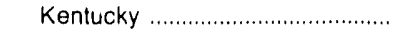 & 37,255 & 31,287 & 40,976 & 27,261 & 152,600 & 139,924 \\
\hline 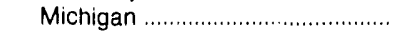 & 244,362 & 200,352 & 102,033 & 87,247 & 78,573 & 50,08 ? \\
\hline 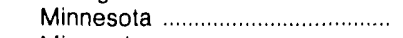 & 183,729 & 188,737 & 46,264 & 43,396 & 41,621 & 36,800 \\
\hline 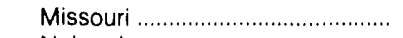 & 26,635 & 19,892 & 69,849 & 38,886 & 38,594 & 33,430 \\
\hline 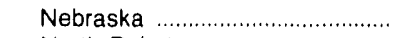 & 8,355 & 10,456 & 12,554 & 9,561 & 5,116 & 5,046 \\
\hline 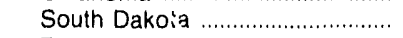 & 38,625 & 37,803 & 14,495 & 9,253 & 8,409 & 5,144 \\
\hline 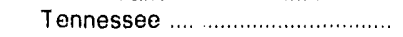 & 9,476 & 10,270 & 46,311 & 27,900 & 42,657 & 31,685 \\
\hline 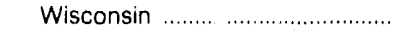 & 273,650 & 226,563 & 75,758 & 84,664 & 29,508 & 21,416 \\
\hline 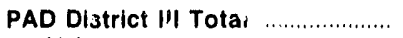 & 3,956 & 3,050 & 395,649 & 326,243 & 434,962 & 432,914 \\
\hline 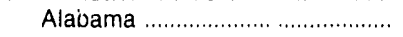 & 1,773 & 1,442 & 47,236 & 51,571 & 88,349 & 81,243 \\
\hline 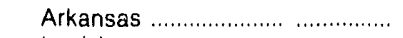 & 55 & 32 & 10,684 & 18,475 & 20,300 & 22,918 \\
\hline 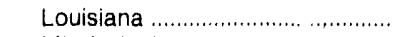 & 99 & 323 & 54,428 & 35,497 & 36,289 & 24,230 \\
\hline Mississippi ............................... & 180 & 273 & 25,175 & 35,915 & 34,130 & 23,570 \\
\hline 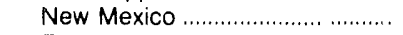 & 514 & 441 & 23,573 & 21,249 & 71,161 & 44,243 \\
\hline 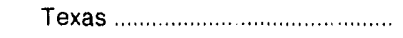 & 1,334 & 539 & $22,4,553$ & 163,537 & 184,732 & 236,711 \\
\hline PAD District IV Total ....................... & 44,722 & 49,637 & 109,842 & 75,564 & 171,297 & 197,695 \\
\hline 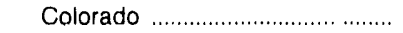 & 2,221 & 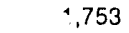 & 42,770 & 22,623 & 45,199 & 35,260 \\
\hline 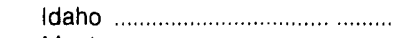 & 25,838 & 23,431 & 18,114 & 14,624 & 20,019 & 30,087 \\
\hline 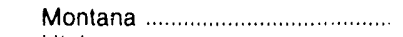 & 9,850 & 15,377 & 8,361 & 8,558 & 18,124 & 45,880 \\
\hline 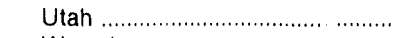 & 5,520 & 7,680 & 29,284 & 19,280 & 40,870 & 32,114 \\
\hline 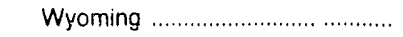 & 1,293 & 1,396 & 11,315 & 10,479 & 47,085 & 54,353 \\
\hline PAD District $\vee$ Total ....................... & 280,499 & 279,280 & 541,685 & 397,644 & 410,107 & 428,039 \\
\hline Alaska & 53,971 & 63,744 & 36,760 & 34,630 & 27,629 & 35,482 \\
\hline Arizona & 264 & $\angle B 3$ & 20,391 & 15,715 & 37,327 & 59,550 \\
\hline 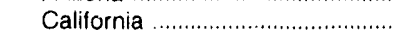 & 9,580 & 10,108 & 265,230 & 193,766 & 215,198 & 168,271 \\
\hline 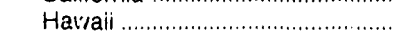 & 74 & 17 & 25,357 & 20,800 & 4,738 & 4,090 \\
\hline Nevada & 12,216 & 10,587 & 19,107 & 15,896 & 57,528 & 94.996 \\
\hline Oregon & 77,372 & 79,305 & 63,832 & 45,131 & 29,093 & 27,587 \\
\hline 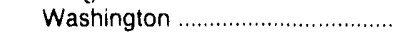 & 127,023 & 115,235 & 111,008 & 71,707 & 38.595 & 38,062 \\
\hline
\end{tabular}

See footnotes at end of table. 
Table 16. Adjusted Sales of Distillate Fuel Oil by End Use, 1988 and 1989-Continued

(Thousand Gallons)

\begin{tabular}{|c|c|c|c|c|c|c|}
\hline \multirow{2}{*}{ Destination } & \multicolumn{2}{|c|}{ Oll Company } & \multicolumn{2}{|c|}{ Farm } & \multicolumn{2}{|c|}{ Electric Utility } \\
\hline & 1988 & 1989 & 1988 & 1989 & 1988 & 1989 \\
\hline U.S. Total & 872,133 & 845,217 & $3,420,671$ & $3,196,928$ & 800,085 & $1,078,887$ \\
\hline PAn District I Total & 35,160 & 38,334 & 403,743 & 398,098 & 420,314 & 627,397 \\
\hline St'bdistrict IA Total & 1,492 & 1,832 & 21,679 & 18,981 & 30,371 & 39,534 \\
\hline Connecticut ...................................... & 240 & 286 & 1,905 & 2,520 & 2,405 & 5,386 \\
\hline 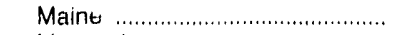 & 11 & 403 & 6,112 & 4,540 & 606 & 2,915 \\
\hline 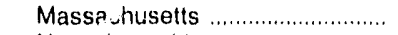 & 853 & 763 & 2,447 & 2,910 & $2<, 055$ & 24,448 \\
\hline New' Hampshire ........................... & 63 & 107 & 2,303 & 1,961 & 1,233 & 3,306 \\
\hline 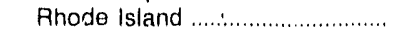 & 300 & 249 & 232 & 199 & 2,164 & 1,961 \\
\hline 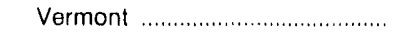 & 25 & 23 & 8,679 & 6,851 & 1,908 & 1,517 \\
\hline 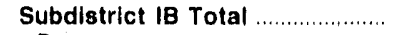 & 27,795 & 31,843 & 152,663 & 144,281 & 270,705 & 401,417 \\
\hline 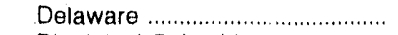 & 380 & 269 & 6,477 & 5,541 & 6,261 & 14,312 \\
\hline 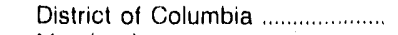 & - & - & - & - & 71,720 & 71,970 \\
\hline 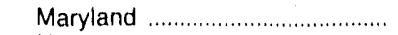 & 843 & 968 & 24,984 & 23,920 & 16,697 & 63,558 \\
\hline 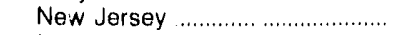 & 4,946 & 4,236 & 16,849 & 19,113 & 33,155 & 54,808 \\
\hline 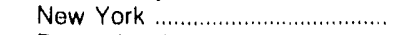 & 7,506 & 10,284 & 50,739 & 48,870 & 81,982 & 114,115 \\
\hline 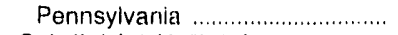 & 14,121 & 16,087 & 33,615 & 46,838 & 60,890 & 82,654 \\
\hline Subdistrict IC Total & 5,873 & 4,658 & 249,401 & 234,835 & 119,238 & 186,446 \\
\hline Florida & 938 & 979 & 83,471 & 47,533 & 56,055 & 79,629 \\
\hline 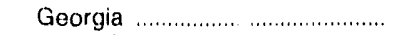 & 156 & 280 & 66,123 & 82,966 & 12,310 & 16,155 \\
\hline Nortn Carolina & 22 & 81 & 42,810 & 37,584 & 18,434 & 22,419 \\
\hline South Carolina ................................. & 692 & 299 & 21,478 & 27,394 & 8,692 & 11,880 \\
\hline 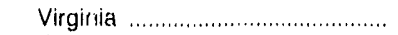 & 1,191 & 1,088 & 29,428 & 32,398 & 17,231 & 33,892 \\
\hline 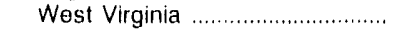 & 2,873 & 1,931 & $5,0 \Omega i$ & 4,960 & 6,316 & 22,472 \\
\hline PAD District II Total .......................... & 58,243 & 60,550 & $1,525,354$ & $1,393,420$ & 161,038 & 188,359 \\
\hline 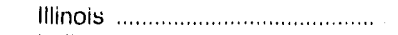 & 7,540 & 6,078 & 189,073 & 170,578 & 29,615 & 24,651 \\
\hline 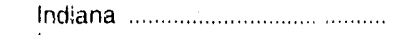 & 1,289 & 930 & 85,050 & 84,188 & 19,048 & 29,115 \\
\hline 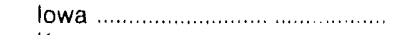 & 55 & 71 & 175,643 & 147,584 & 8,624 & 4,832 \\
\hline 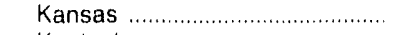 & 7,730 & 8,024 & 146,670 & 132,404 & 6,280 & 7.468 \\
\hline 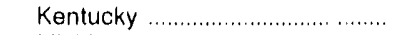 & 574 & 683 & 44,062 & 38,602 & 8,740 & 17,107 \\
\hline 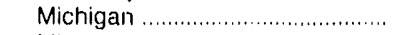 & 3,878 & 1,353 & 75,242 & $59,83 \mathrm{C}$ & 34,125 & 37,769 \\
\hline Minnesota .................................... & 512 & 524 & 154,086 & 129,423 & 8,439 & 6,384 \\
\hline 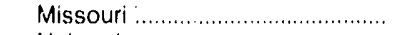 & 465 & 315 & 75,031 & 79,324 & 9,594 & 9,620 \\
\hline Nebraska & 458 & 22 & 159,059 & 145,637 & 647 & 3,902 \\
\hline 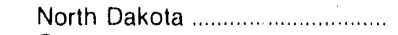 & 2,686 & 4,421 & 82,674 & 89,892 & 1,999 & 4,302 \\
\hline Ohio & 7,547 & 6,153 & 75,940 & 74,818 & 20,205 & 21,269 \\
\hline 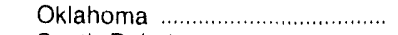 & 23,790 & 30,983 & 51,635 & 41,123 & 66 & 542 \\
\hline South Dakota ................................ & 11 & 12 & 91,500 & 74,497 & 1,265 & 862 \\
\hline 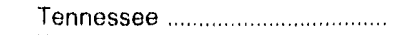 & 984 & 551 & 37,538 & 36,97 & 2,842 & 3,447 \\
\hline Wisconsin & 724 & 430 & 82,151 & 85,923 & 9,551 & 17,090 \\
\hline PAD District III Total & 671,714 & 675,879 & 904,614 & 740,010 & 71,653 & 114,885 \\
\hline 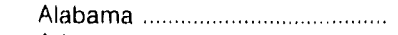 & 2,159 & 9,997 & 37,124 & 60,320 & 7,208 & 10,486 \\
\hline 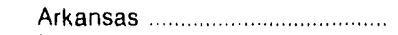 & 1,160 & 4,913 & 153,512 & 92,106 & 11,103 & 11,073 \\
\hline 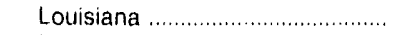 & 301,589 & 328,594 & 177,694 & 91,754 & 13,349 & 20,371 \\
\hline 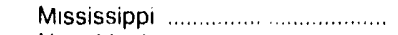 & 40,260 & 18,459 & 122,241 & 133,238 & 4,361 & 5,072 \\
\hline 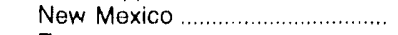 & 16,701 & 11,533 & 26,954 & 16,925 & 387 & 6,351 \\
\hline 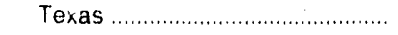 & 309,844 & 302,383 & 387,089 & 345,675 & 35,245 & 61,532 \\
\hline PAD District IV Total ......................... & 20,442 & 20,914 & 192,693 & 196,150 & 14,497 & 18,933 \\
\hline 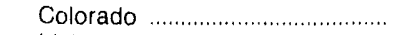 & 4,329 & 1,316 & 62,193 & 54,657 & 3,088 & 5,161 \\
\hline 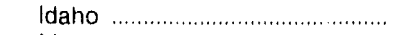 & 93 & 8 & 66,078 & 68,266 & 48 & 27 \\
\hline 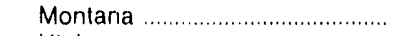 & 1,032 & 1,093 & 37,452 & 53,859 & 1,043 & 2,552 \\
\hline 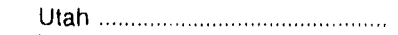 & 1.555 & 4,459 & 16,783 & 9,656 & 4,386 & 4,293 \\
\hline 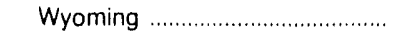 & 13,434 & 14,038 & 10,187 & 9,713 & 5,931 & 6,899 \\
\hline FAD District $\vee$ Total $\ldots \ldots \ldots$ & 86,573 & 49,540 & 394,267 & 469,243 & 132,584 & 129,313 \\
\hline Alaska & 42,770 & 27,330 & 358 & 257 & 34,863 & 21,953 \\
\hline - & 231 & 67 & 22,433 & 31,647 & 4,993 & 4,382 \\
\hline California . .................................... & 40.105 & 19,399 & 254,767 & 302,124 & 28,521 & 19,719 \\
\hline 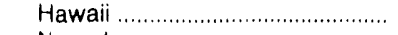 & 1,782 & 923 & 18,509 & 11,132 & 60,862 & 74,240 \\
\hline 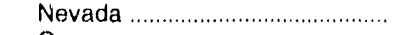 & 165 & 321 & 8,360 & 5,187 & 1,768 & 2,639 \\
\hline Oregon & 620 & 279 & 49,234 & 49,245 & 467 & 5,363 \\
\hline 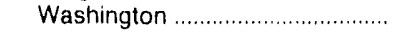 & 900 & 1,222 & 40,608 & 69,650 & 1,110 & 1,017 \\
\hline
\end{tabular}

See footnotes at end of table. 
Table 16. Adjusted Sales of Distillate Fuel Oil by End Use, 1988 and 1989-Continued (Thousand Gallons)

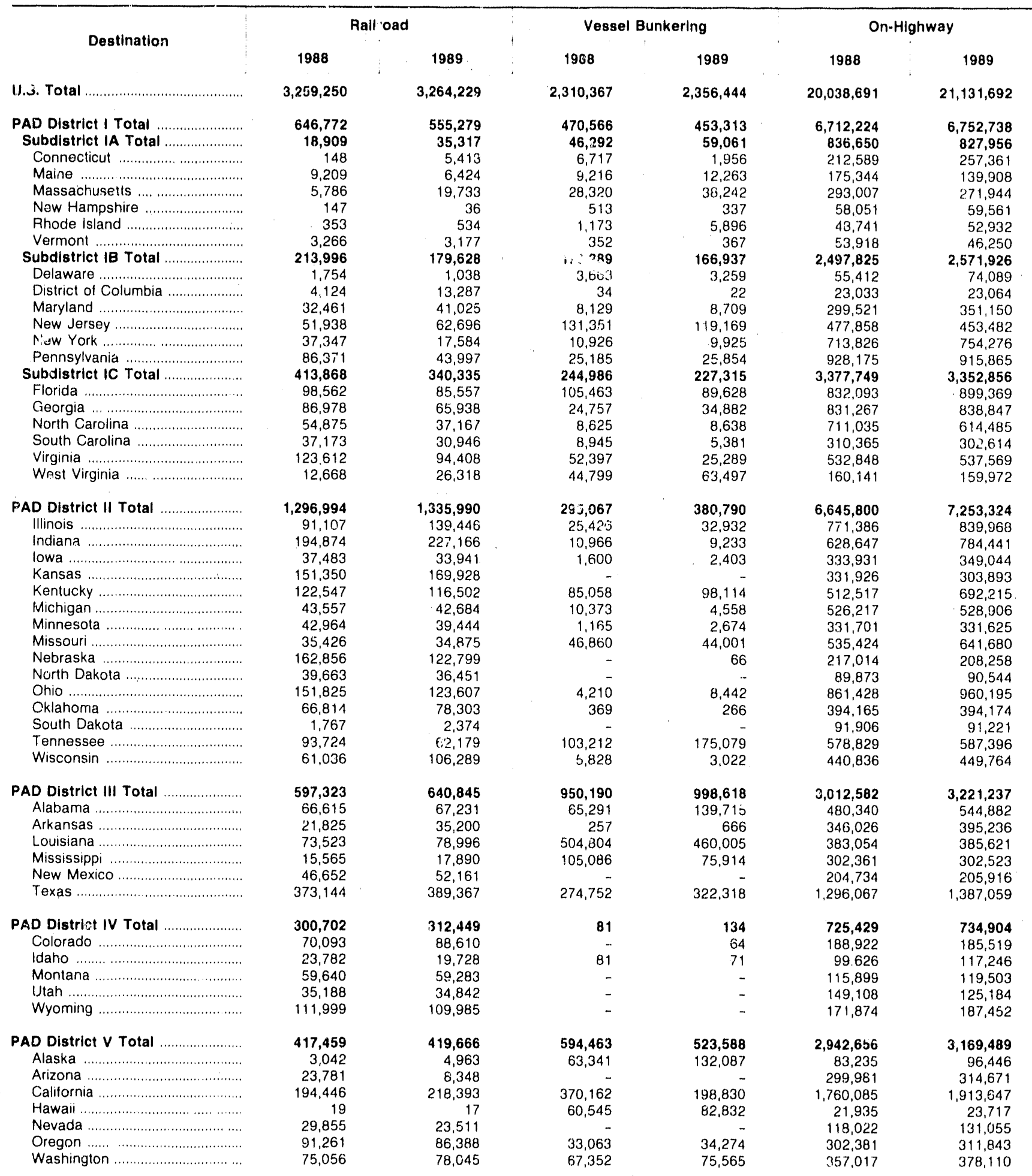

See footnotes at end of table. 
Table 16. Adjusted Sales of Distillate Fuel Oil by End Use, 1988 and 1989-Continued

(Thousand Gallons)

\begin{tabular}{|c|c|c|c|c|c|c|c|c|}
\hline \multirow{2}{*}{ Destination } & \multicolumn{2}{|c|}{ Milltary } & \multicolumn{2}{|c|}{ Off-Highway } & \multicolumn{2}{|c|}{ All Other } & \multicolumn{2}{|c|}{ Total } \\
\hline & 1988 & 1989 & 1988 & 1989 & 1988 & 1989 & 1988 & 1989 \\
\hline$\ldots \ldots \ldots \ldots \ldots \ldots \ldots \ldots \ldots$ & 980,877 & 928,316 & $1,821,819$ & $1,637,497$ & 66,763 & 29,106 & $47,985,420$ & $48,393,450$ \\
\hline PAD District I Total & 179,235 & 141,367 & 415,066 & 461,637 & 15,811 & 11,307 & $18,570,384$ & $18,773,874$ \\
\hline Subdistrict |A Total .......................... & 13,837 & 15,102 & 64,179 & 66,502 & 674 & 597 & $3,762,185$ & $4,000,264$ \\
\hline 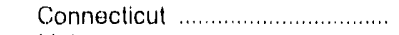 & 1,305 & 611 & 12,010 & 18,713 & 281 & 480 & 957,661 & $1,073,210$ \\
\hline Maine & 2.304 & 2,984 & 20,028 & 12,836 & - & 39 & 574,305 & 516,210 \\
\hline Massachusetts ……....................... & 3,850 & 1,988 & 17,627 & 18,219 & 66 & . & $1,501,415$ & $1,650,549$ \\
\hline New Hampshire ........................... & 75 & 1,542 & 6.110 & 7,674 & - & - & 284,329 & 318,155 \\
\hline Rhode Island & 5,682 & 7,748 & 999 & 3,344 & 185 & 79 & 249,646 & 248,361 \\
\hline Vermont ........... & 620 & 229 & 7,404 & 5,717 & $1: ?$ & - & 194,829 & 193,778 \\
\hline 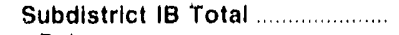 & 36,071 & 55,629 & 134,658 & 180,995 & $3,2,86$ & 3,338 & $8,581,098$ & $8,807,488$ \\
\hline 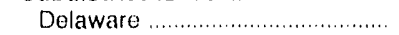 & 33 & 85 & 3,384 & 4,202 & 94 & - & 160,736 & 179,727 \\
\hline 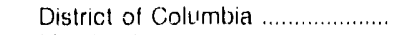 & 8,836 & 2,996 & 81 & 31 & - & - & 143,240 & 140,261 \\
\hline 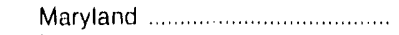 & 3,930 & 22,336 & 15,272 & 32,165 & 2,710 & 2,569 & 759,648 & 875,137 \\
\hline 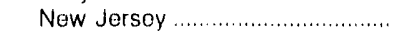 & 6,008 & 9,124 & 28,363 & 52,452 & 32 & 48 & $1,900,310$ & $1,856,878$ \\
\hline New lork & 12,611 & 10,410 & 41,963 & 40,298 & 263 & 3 & $3,159,737$ & $3,178,401$ \\
\hline 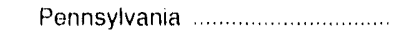 & 4,653 & 10,677 & 45,595 & 51,847 & 187 & 718 & $2,457,425$ & $2,577,083$ \\
\hline Subdistrict IC Total & 129,327 & 70,636 & 216,230 & 214,139 & 11,851 & 7,371 & $6,227,101$ & $5,966,123$ \\
\hline Florida ………………………........ & 28,773 & 20,305 & 56,154 & 65,627 & 1,489 & 1,307 & $1,457,996$ & $1,461,804$ \\
\hline$\ldots+\ldots+\ldots+\ldots+\ldots \ldots \ldots$ & 12,085 & 9,153 & 35,397 & 38,179 & 4,165 & 1,610 & $1,171,711$ & $1,183,830$ \\
\hline North Carolina ................................ & 4,308 & 7,794 & 28,443 & 26,655 & 2,780 & 2,234 & $1,323,790$ & $1,135,279$ \\
\hline South Cardlina ............................... & 3.221 & 2,557 & 24,928 & 26,601 & 1,277 & 908 & 529,879 & 526,128 \\
\hline 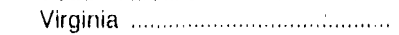 & 80,442 & 30,664 & 53,024 & 36,720 & 2,112 & 1,299 & $1,371,623$ & $1,230,064$ \\
\hline 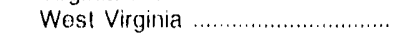 & 498 & 163 & 18,285 & 20,358 & 28 & 13 & 372,102 & 429,017 \\
\hline PAD Districf H Total & 50,752 & 44,570 & 414,244 & 340,350 & 40,819 & 13,925 & $13,238,862$ & $13,411,944$ \\
\hline Illinois . . & 1,879 & 4,430 & 56,630 & 37,553 & 3,544 & 161 & $1,419,887$ & $1,457,021$ \\
\hline 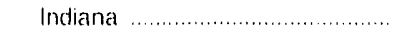 & 4,031 & 6,569 & 17,954 & 29,465 & 3,198 & 2,819 & $1,210,126$ & $1,404,487$ \\
\hline 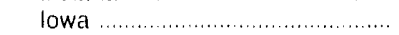 & 110 & 272 & 17,335 & 9,595 & 2,848 & 1,876 & 673,021 & 628,372 \\
\hline 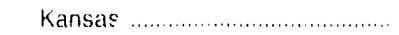 & 1,947 & 855 & 17,127 & 12,352 & 1,518 & 1,601 & 696,175 & 662,271 \\
\hline 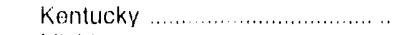 & 10,793 & 5,419 & 21,276 & 21,811 & 719 & 10 & $1,037,117$ & $1,188,945$ \\
\hline Michigan. & 3,222 & 3,146 & 49,716 & 45,397 & 2,192 & 1,744 & $1,173,490$ & $1,063,066$ \\
\hline 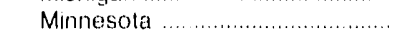 & 2,704 & 2,030 & 23,638 & 26,163 & 2,122 & 1,818 & 838,945 & 809,017 \\
\hline 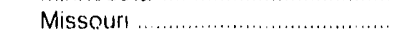 & 6,309 & 4,994 & 37,120 & 21,499 & 6,791 & 1,165 & 888,098 & 929,681 \\
\hline Nobraska & 1,423 & 1.063 & 18,094 & 17,083 & - & -. & 585,578 & 523,895 \\
\hline North Dakota & 2,505 & 1,344 & 5,470 & 4,509 & 76 & 194 & 284,583 & 295,626 \\
\hline Ohio ……………………… & 9,006 & 8,591 & 27,752 & 27,963 & 358 & 335 & $1,553,942$ & $1,605,659$ \\
\hline Oklahoma …................................ & 2,412 & 1,491 & 28,108 & 22,047 & 4,616 & 227 & 624,037 & 618,234 \\
\hline South Dakola ................................... & 730 & 512 & 11,684 & 5,642 & 336 & 540 & 260,730 & 227,860 \\
\hline 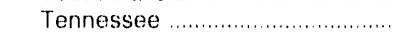 & 1,054 & 2,785 & 59,940 & 26,619 & 1,236 & 650 & 977,804 & 968,157 \\
\hline Wisconsin & 2,626 & 1,064 & 22,398 & 32,652 & 11,264 & 776 & $1,015,329$ & $1,029,655$ \\
\hline PAD District III Total & $407, j 84$ & 442,179 & 505,034 & 376,969 & 8,780 & 3,425 & $7,964,040$ & $7,976,262$ \\
\hline Alabama & 4,853 & 6,335 & 53,110 & 63,870 & 3,578 & 1,999 & 857,636 & $1,039,092$ \\
\hline 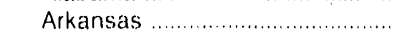 & 2,467 & 2,069 & 30,943 & 32,128 & 2,793 & 63 & 601,125 & 614,878 \\
\hline Louisiana ......................................... & 20,955 & 83,075 & 74,854 & 54,974 & 297 & 275 & $1,640,937$ & $1,563,715$ \\
\hline Mississippi ........................................ & 116,607 & 73,439 & 33,755 & 33,576 & 880 & 162 & 800,602 & 720,030 \\
\hline 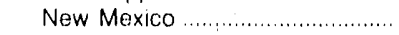 & 1,982 & 912 & 35,167 & 21,484 & 0 & 48 & 427,725 & 381,263 \\
\hline 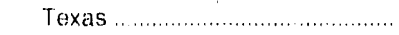 & 260,820 & 276,348 & $277,20 \%$ & 170,936 & 1,232 & 879 & $3,636,015$ & $3,657,284$ \\
\hline PAD District IV Total ................... & 15,584 & 13,748 & 115,045 & 93,611 & 410 & 450 & $1,710,744$ & $1,714,188$ \\
\hline Colorado & 8,482 & 8,353 & 43,198 & 27,355 & 33 & 46 & 470,526 & 430,717 \\
\hline Idaho & 2,542 & 738 & 17,004 & 13,872 & - & 26 & 273,223 & 288,124 \\
\hline Montana ................................ & 480 & 351 & 11,171 & 15,989 & 213 & 25 & 263,266 & 322,470 \\
\hline Utah & 3,797 & 4,120 & 21,284 & 18,551 & - & - & 307,776 & 260,179 \\
\hline 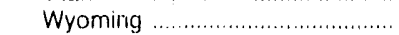 & 283 & 186 & 22,388 & 17,844 & 165 & 353 & 395,953 & 412,697 \\
\hline PAD District $\vee$ rotal & 327,723 & 286,451 & 372,431 & 364,928 & 943 & - & $6,501,390$ & $6,517,182$ \\
\hline Alaska ………......................... & 17,561 & 34,075 & 13,855 & 13,980 & 40 & - & 377,423 & 464,948 \\
\hline Arizona ….............................. & 1,324 & 2,253 & 25,285 & 37,978 & - & .- & 436,010 & 474,894 \\
\hline California ……........................ & 221,645 & 205,471 & 208,735 & 193,260 & 811 & - & $3.569,284$ & $3,442,990$ \\
\hline 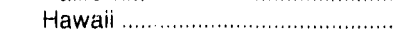 & 54,672 & 31,149 & $7,22.7$ & 5,428 & - & .. & 255,720 & 254,345 \\
\hline Nevada ............................................ & 4,928 & 4,401 & 32,792 & 24,066 & 33 & - & 284,773 & 312,658 \\
\hline 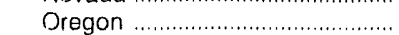 & 1,390 & 733 & 43,412 & 44,577 & - & - & 692,124 & 684,725 \\
\hline Washington ................................... & 26,205 & 8,370 & 41,125 & 45,640 & 59 & - & 886,056 & 882,623 \\
\hline
\end{tabular}

Dashes (-) No data reported.

Nole: Totals may not equal sum of components due to independent rounding.

Sources: - Energy Information Administration Form ElA-821, "Annual Fuel Oil and Kerosene Sales Report," for 1988 and 1989.

- Electric Utility data are based on data obtained on Form EIA-759, "Monthly Power Plant Report," and Form FERC-423, "Monthly Report of Cost and Quality of Fuels for Elestric Plants."

- Railroad data for distillate fuel oil are based on Association of American Railroads' statistics for railroads of Class I and Amtrak Companies.

- On-Highway Diesel data are Federal Highway Administation statistics of highway special fuels use.

- Energy Information Administration's "Petroleum Supply Arnual," Volume 1, 1988 and 1989, (DOE/EIA-0340(88)/1 - (89)/1). 
Table 17. Adjusted Sales of Residual Fuel Oil by End Use, 1988 and 1989

(Thousand Gallons)

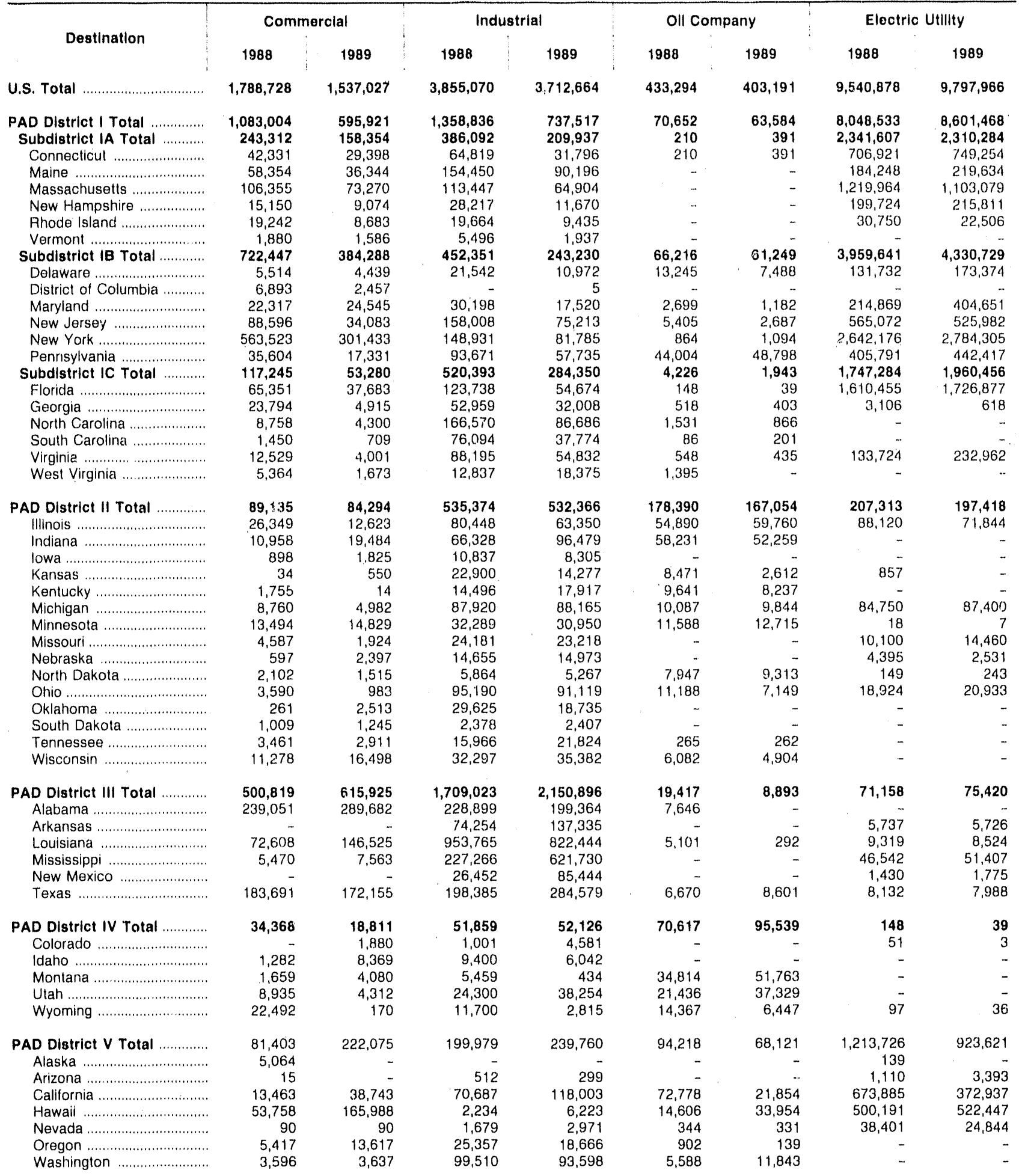

See footnotes at end of table. 
Table 17. Adjusted Sales of Residual Fuel Oil by End Use, 1988 and 1989-Continued

(Thousand Gallons)

\begin{tabular}{|c|c|c|c|c|c|c|c|c|c|c|}
\hline \multirow[b]{2}{*}{ Destination } & \multicolumn{2}{|c|}{ Rallroad } & \multicolumn{2}{|c|}{ Vessel Bunkering } & \multicolumn{2}{|c|}{ Milltary } & \multicolumn{2}{|c|}{ All Other } & \multicolumn{2}{|c|}{ Total } \\
\hline & 1988 & 1989 & 1988 & 1989 & 1988 & 1989 & 1988 & 1989 & 1988 & 1989 \\
\hline 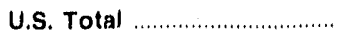 & 33 & 151 & $5,248,981$ & $5,410,263$ & E 137,287 & 105,255 & 175,279 & 36,298 & $21,179,550$ & $21,002,814$ \\
\hline PAD District I Total ................ & - & - & 933,547 & $955,48 \dagger$ & 105,464 & 90,853 & 3,304 & 84 & $11,603,340$ & $11,044,908$ \\
\hline 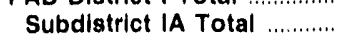 & - & - & 34,404 & 34,218 & 23,830 & 25,823 & - & - & $3,029,455$ & $2,739,007$ \\
\hline 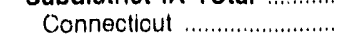 & . & - & 876 & 236 & 4,924 & 3,097 & - & - & 820,082 & 814,172 \\
\hline Maine & - & - & 12,270 & 3,962 & - & 4,085 & - & - & 409,322 & 354,221 \\
\hline 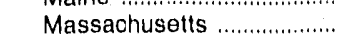 & - & - & 16,311 & 29,557 & 5,785 & 14,388 & - & - & $1,461,862$ & $1,285,197$ \\
\hline New Hampshire .................. & - & - & 445 & - & 6,363 & 1,049 & - & - & 249,899 & 237,604 \\
\hline 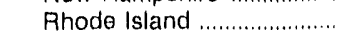 & - & - & 4,501 & 464 & 6,758 & 2,850 & - & - & 80,914 & 43,937 \\
\hline Vermont ................................. & - & - & - & - & - & 354 & - & - & 7,376 & 3,876 \\
\hline Subdistrict IB Total ............... & - & - & 548,917 & 524,719 & 47,454 & 40,918 & 2,945 & - & $5,799,972$ & $5,585,132$ \\
\hline 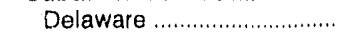 & - & - & 25,630 & 28,740 & - & - & - & - & 197,663 & 225,013 \\
\hline District of Columbia ............. & - & - & - & - & 499 & 2,115 & - & - & 7,392 & 4,578 \\
\hline Maryland ................................ & - & - & 62,820 & 64,120 & 23,654 & 23,688 & - & - & 356,558 & 535,705 \\
\hline New Jersey ........................... & - & - & 220,204 & 288,946 & 6,920 & 2,892 & 60 & - & $1,044,265$ & 929,804 \\
\hline Subdistrict IC Total ........... & - & - & 350,226 & 396,544 & 34,180 & 24,112 & 360 & 84 & $2,773,913$ & $2,720,769$ \\
\hline Florida & - & - & 235,237 & 257,318 & 9,476 & 7,460 & 173 & - & $2,044,577$ & $2,084,050$ \\
\hline Georgia & - & - & 12,092 & 20,346 & 1,783 & 1,947 & - & - & 94,252 & 60,236 \\
\hline North Carolina ..................... & - & - & 5,898 & 19,099 & 10,411 & 4,214 & 187 & 84 & 193,355 & 115,249 \\
\hline South Carolina ........................ & - & - & 19,391 & 17,892 & 5,360 & 6,343 & - & - & 102,381 & 62,919 \\
\hline 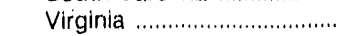 & - & - & 77,609 & 81,889 & 7,149 & 4,149 & - & - & 319,753 & 378,267 \\
\hline West Virginia ......................... & - & - & - & - & - & - & - & - & 19,596 & 20,048 \\
\hline PAD District \| Total .. & 33 & 1 & 24,593 & 18,710 & 4,921 & 4,291 & 248 & 549 & $1,040,004$ & $1,004,682$ \\
\hline 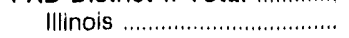 & - & - & 15,351 & 2,671 & 155 & - & 40 & - & 265,353 & 210,249 \\
\hline 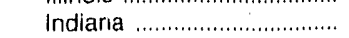 & - & - & 6,831 & 9,908 & - & - & - & - & 142,348 & 178,130 \\
\hline 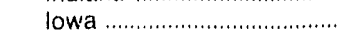 & - & 1 & - & - & - & 10 & - & - & 11,735 & 10,140 \\
\hline 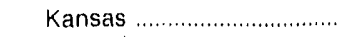 & - & - & - & - & - & - & - & - & 32,263 & 17,439 \\
\hline 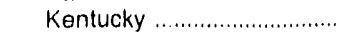 & - & - & - & - & - & - & - & - & 25,892 & 26,168 \\
\hline 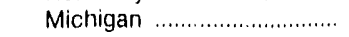 & - & - & 1,315 & 5,397 & - & - & - & 74 & 192,832 & 195,862 \\
\hline Minnesota .............................. & 11 & - & 306 & 73 & - & - & 190 & 153 & 57,895 & 58,726 \\
\hline Missouri ................................ & 22 & - & - & - & 4,218 & 3,709 & - & - & 43,107 & 43,311 \\
\hline 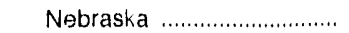 & - & - & - & - & - & - & 16 & 19 & 19,664 & 19,920 \\
\hline South Dakota ...................... & - & - & - & - & - & 2 & - & - & 3,387 & 3,654 \\
\hline 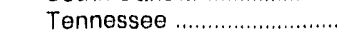 & - & - & 66 & 22 & 548 & 571 & - & - & 20,306 & 25,590 \\
\hline 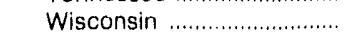 & - & - & 200 & 187 & - & - & - & - & 49,857 & 56,971 \\
\hline PAD District III Total ............... & - & - & $1,794,626$ & $1,897,207$ & 4,292 & 1,229 & 159,381 & - & $4,258,716$ & $4,749,570$ \\
\hline Alabama & - & .. & 74,707 & 97,958 & 28 & 1,229 & - & - & 550,331 & 588,234 \\
\hline 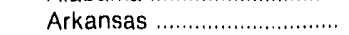 & - & - & - & - & - & - & ـ & - & 79,991 & 143,061 \\
\hline 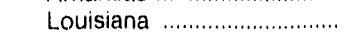 & - & - & 847,838 & 845,590 & 4,264 & - & 12,636 & - & $1,905,531$ & $1,823,375$ \\
\hline 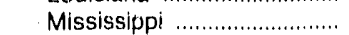 & - & $\sim$ & 64,215 & 42,127 & - & - & - & - & 343,492 & 722,827 \\
\hline New Mexico ............................ & - & - & - & - & - & - & - & - & 27,882 & 87,219 \\
\hline 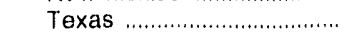 & - & - & 807,866 & 911,532 & - & - & 146,745 & - & $1,351,488$ & $1,384,854$ \\
\hline PAD District IV Total ............... & - & 129 & - & - & - & - & 172 & 1,524 & 157,164 & 168,168 \\
\hline Colorado .................................. & - & ... & $\sim$ & - & - & - & - & - & 1,051 & 6,464 \\
\hline 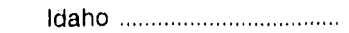 & - & - & - & - & - & - & - & - & 10,682 & 14,411 \\
\hline 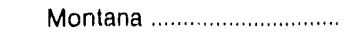 & - & - & - & - & - & - & - & 1,331 & 41,932 & 57,608 \\
\hline 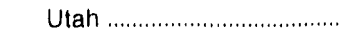 & - & 58 & - & - & - & - & - & - & 54,670 & 79,952 \\
\hline 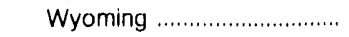 & - & 72 & - & - & - & - & 172 & 193 & 48,829 & 9,732 \\
\hline PAD District $\vee$ Total ................ & - & 21 & a $2,496,215$ & $2,538,865$ & 22,610 & 8,882 & 12,175 & 34,141 & $4,120,326$ & $4,035,486$ \\
\hline 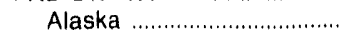 & - & - & 5,624 & 4,313 & - & - & - & - & 10,828 & 4,313 \\
\hline 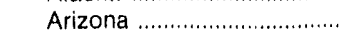 & - & - & - & - & - & - & - & - & 1,636 & 3,692 \\
\hline 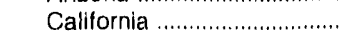 & - & - & $1,878,459$ & $1,791,584$ & 7,162 & - & - & - & $2,716,435$ & $2,343,121$ \\
\hline 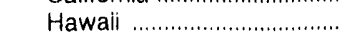 & - & - & 63,078 & 78,996 & 3,191 & 3,647 & 12,083 & 34,069 & 649,142 & 845,324 \\
\hline 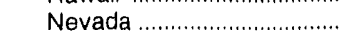 & - & - & - & - & - & - & - & - & 40,513 & 28,236 \\
\hline Oregon & - & 21 & $169,41 T$ & 173,342 & 236 & - & 13 & 72 & 201,342 & 205,857 \\
\hline Washington & - & - & 379,637 & 490,630 & 12,021 & 5,235 & 78 & . & 500,430 & 604,943 \\
\hline
\end{tabular}

a See Technical Note 3 for adjustment to vessel bunkering data.

E Estimated data. Annual estimate is based on eleven months of data.

Dashes $(-)=$ No data reported.

Note: Totals may not equal sum of components due to independent rounding.

Sources: - Energy Information Administration' Form EIA-821, "Annual Fuel Oil and Kerosene Sales Report," for 1988 and 1989.

- Electric Utility data are based on data obtained on Form ElA-759, "Monthly Power Plant Report," and Form FERC-423, "Monthly Report of Cost and Quality of Fuels for Electric Plants."

- Vessel Bunkering data for residual fuel oil are based on ElA estimates of intercoastal bunkering and the Bureau of the Census Report, "United States Foreign Trade, Bunker Fuels."

- Milttary data for residual fuel oil are based on Defense Fuel Supply Center's "DEIS Report."

- Energy Information Administration's "Petroleum Supply Arinual," Volume 1, 1988 and 1989, (DOE/ElA-0340(88)/1 - (89)/1). 
Table 18. Adjusted Sales of Kerosene by End Use, 1988 and 1989 (Thousand Gallons)

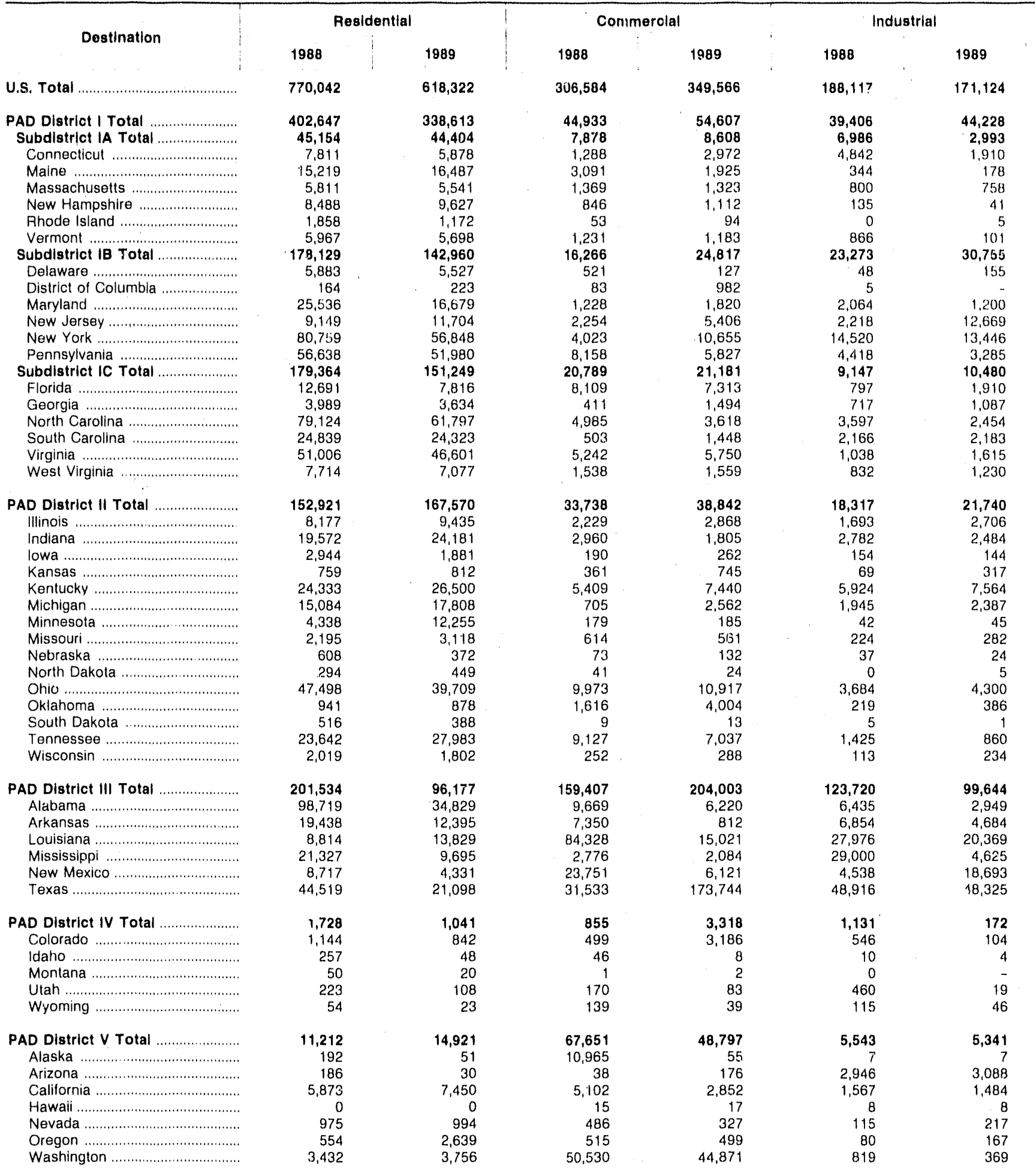

See footnotes at end of table. 
Table 18. Adjusted Sales of Kerosene by End Use, 1988 and 1989-Continued (Thousand Gallons)

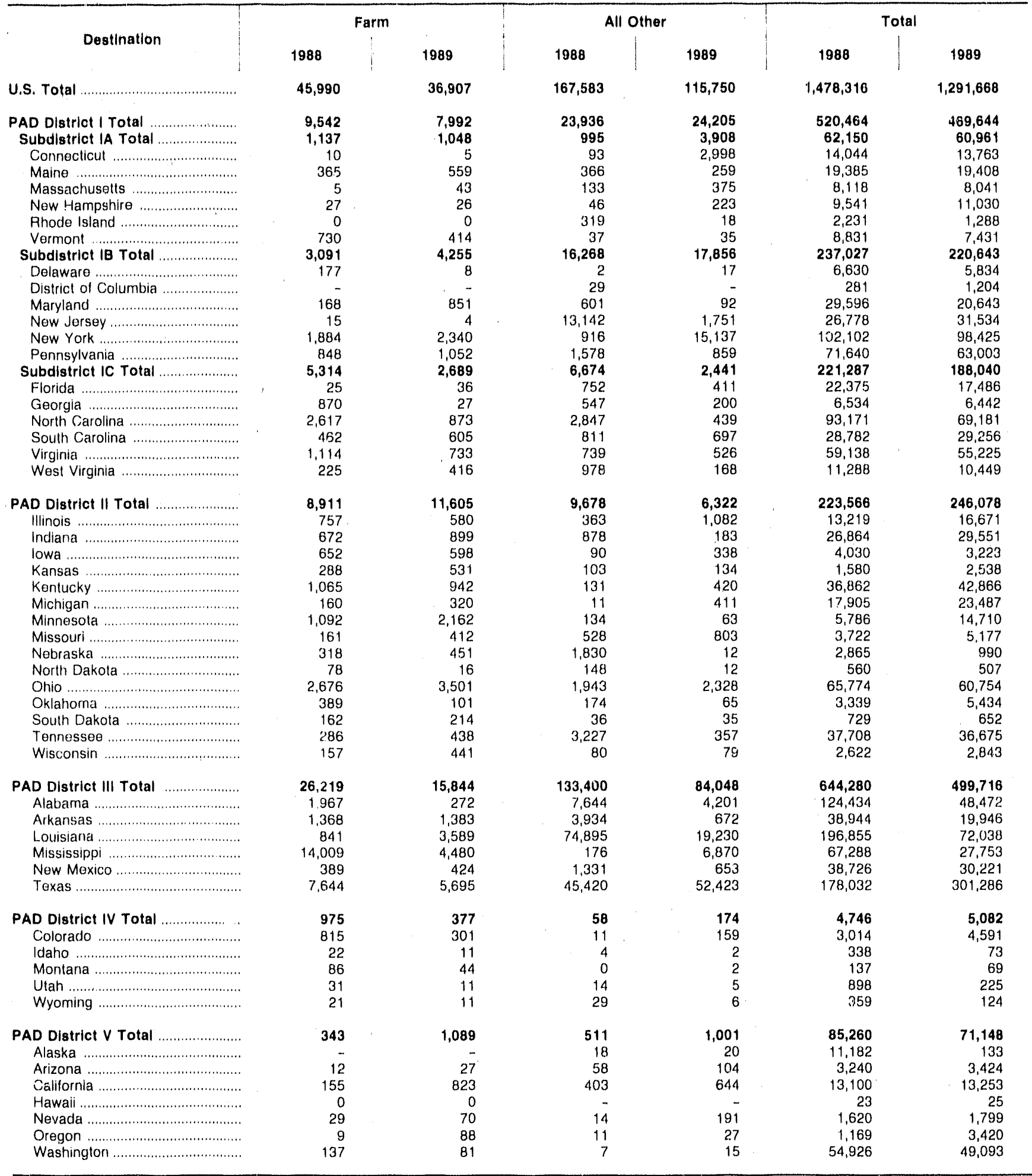

Dashes $(-)$ No data report-1.

Note: Totals may not equal sum of components due to independent rounding.

Sources: - Energy Information Administration Form FlA-821, "Annual Fuel Oil and Kerosene Sales Report," for 1988 and 1989

- Energy Information Administration's "Petroleum Supply Annual," Volume 1, 1988 and 1989, (DOE/EIA.0340(88)/1 - (89)/1). 


\section{Table 19. Adjusted Sales for Residential Use: Distillate Fuel Oil and Kerosene, 1989}

(Thousand Gallons)

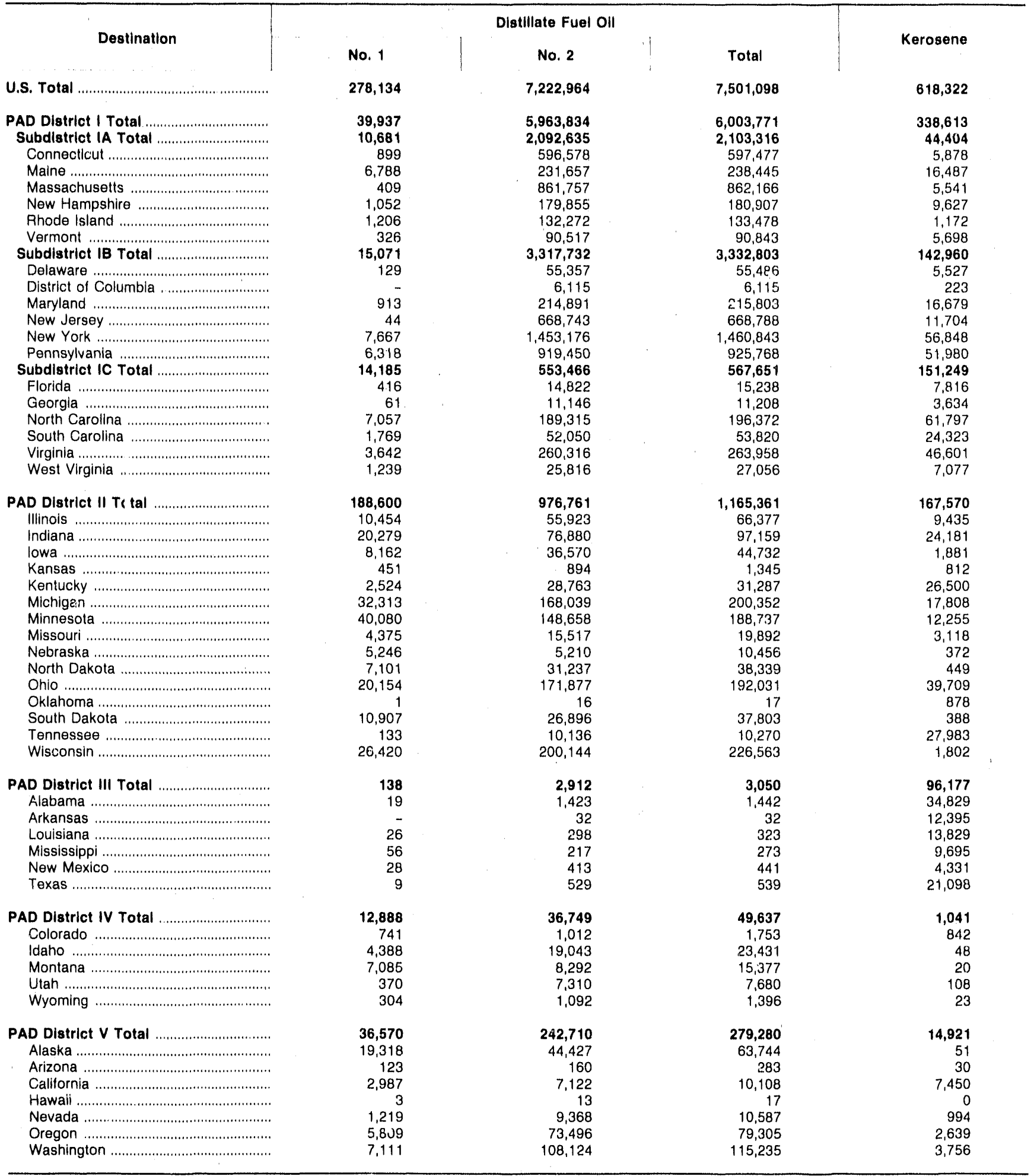

Dashes (-) = No data reported.

Note: Totals may not equal sum of components due to independent rounding

Sources: - Energy Information Administration Form ElA-821, "Annual Fuel Oil and Kerosene Sales Report," for 1989.

- Energy Information Administration's "Petroleum Supply Annual," Volume 1, 1989, (DOE/E/A-0340(89)/1) 
Table 20. Adjusted Sales for Commercial Use: Distillate Fuel Oil, Residual Fuel Oil, and Kerosene, 1989 (Thousand Gallons)

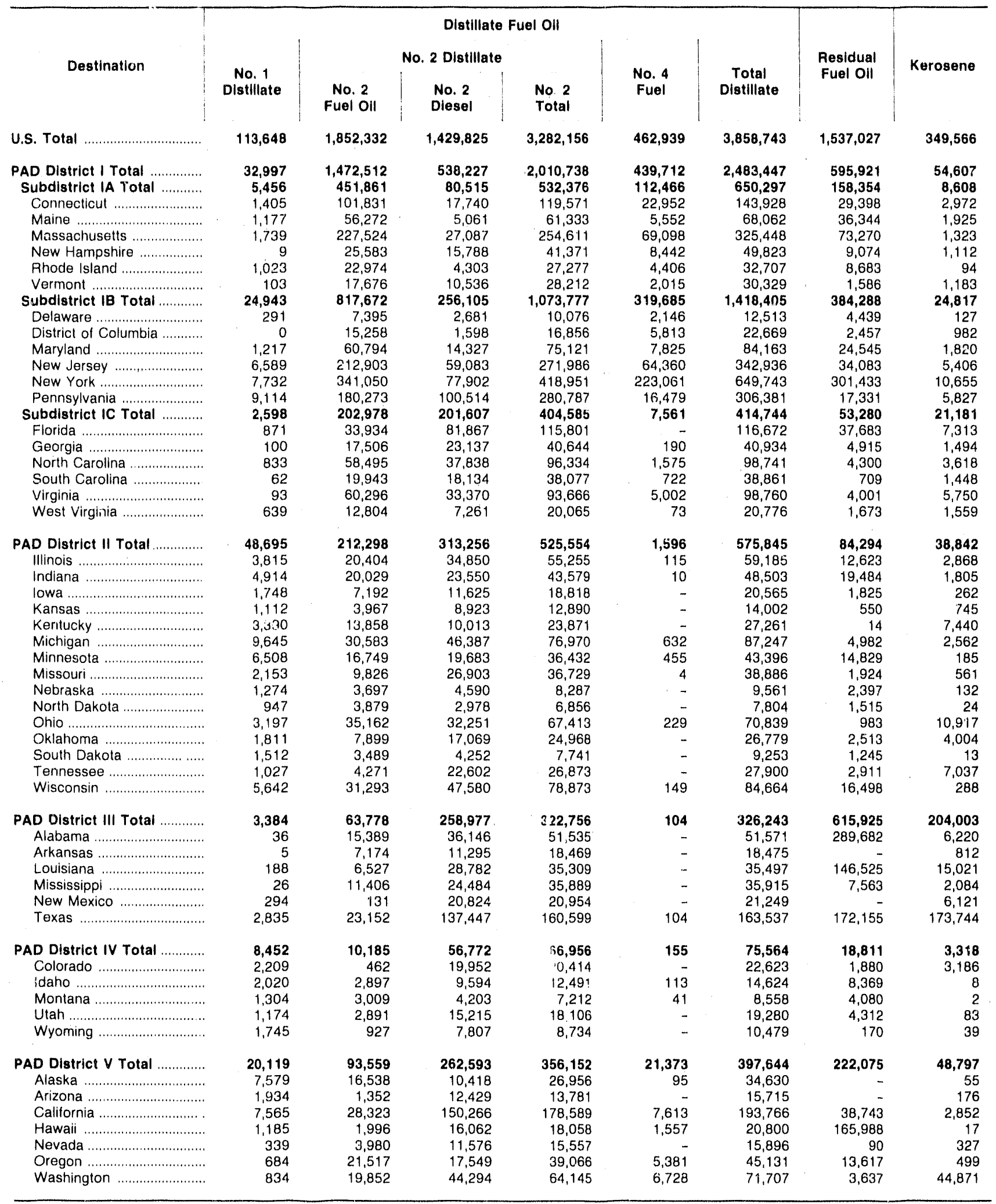

Dashes (-) :.: No data reported.

Note: Totals may not equal sum of components due to independent rounding

Sources: - Energy Information Administration Form ElA-821, "Annual Fuel Oll and Kerosene Sales Report," for 1989.

- Energy Information Administration's "Petroleum Supply Annual," Volume 1, 1989, (DOE/EIA-0340(89)/1). 
Table 21. Adjusted Sales for Industrial and Oil Company Use: Distillate Fuel Oil, Residual Fuel Oil, and Kerosene, 1989

(Thousand Gallons)

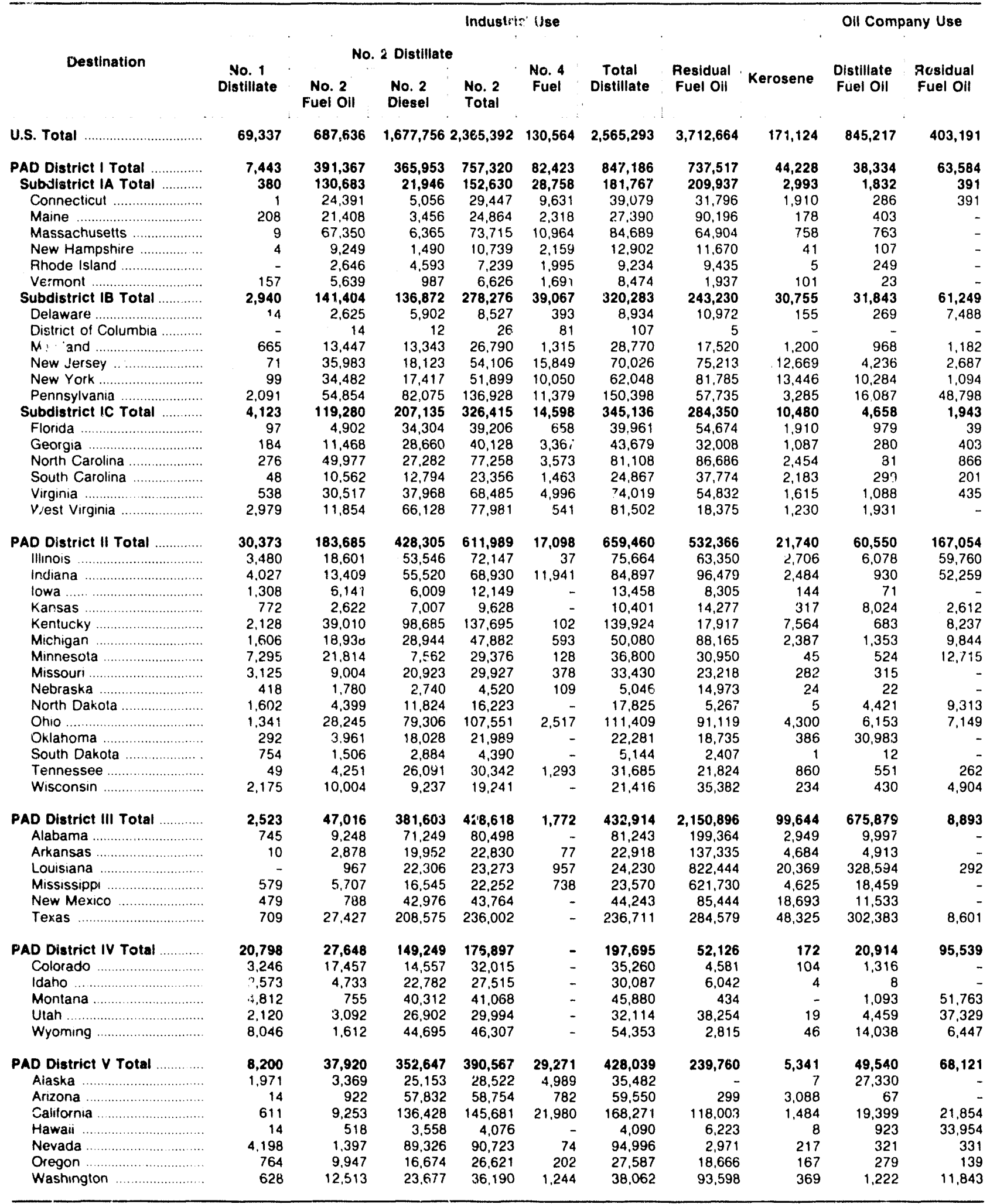

Dashes $(\cdot)=$ No data reported

Note: Totals may not equal sum of components due to independent rounding

Sources: - Eivergy In'ormation Administration Form EIA-821. "Annual Fuel Oil and Kerosene Sales Report," for 1989.

- Energy Information A, dministration's "Petroleum Supply Annual," Volume 1, 1989. (DOE/EIA.0340(89)/1) 
Table 22. Adjusted Sales for Farm Use: Distillate Fuel Oil and Kerosene; Adjusted Sales for Electric Utility Use: Distillate Fuel Oil and Residual Fuel Oil, 1989 (Thousand Gallons)

\begin{tabular}{|c|c|c|c|c|c|c|}
\hline \multirow[b]{2}{*}{ Destination } & \multicolumn{4}{|c|}{ Farm } & \multicolumn{2}{|c|}{ Electric } \\
\hline & Diesel & $\begin{array}{l}\text { Other } \\
\text { Distillate }\end{array}$ & $\begin{array}{l}\text { Total } \\
\text { Distillate }\end{array}$ & Kerosene & $\begin{array}{l}\text { Distillate } \\
\text { Fuel Oll }\end{array}$ & $\begin{array}{l}\text { Residual } \\
\text { Fuel Oll }\end{array}$ \\
\hline 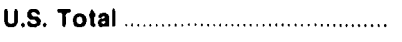 & $3,043,967$ & 152,961 & $3,196,928$ & 36,907 & $1,078,887$ & $9,797,966$ \\
\hline $\begin{array}{l}\text { PAD District I Total } \ldots \ldots \ldots \ldots \ldots \ldots \ldots \\
\text { Subdistrict IA Total }\end{array}$ & $\begin{array}{r}339,053 \\
10,976\end{array}$ & $\begin{array}{r}59,044 \\
8,005\end{array}$ & $\begin{array}{r}398,098 \\
18,981\end{array}$ & $\begin{array}{l}7,992 \\
1,048\end{array}$ & $\begin{array}{r}627,397 \\
39,534\end{array}$ & $\begin{array}{l}8,601,468 \\
2,310,234\end{array}$ \\
\hline 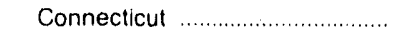 & 1,498 & 1,022 & 2,520 & 5 & 5,386 & 749,254 \\
\hline 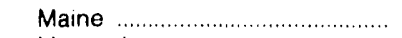 & 3,325 & 1,216 & 4,540 & 559 & 2,915 & 219,634 \\
\hline 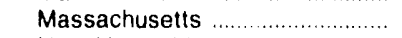 & 1,012 & 1,898 & 2,910 & 43 & 24,448 & $1,103,079$ \\
\hline New Hampshire ............................ & 742 & 1,219 & 1,961 & 26 & 3,306 & 215,811 \\
\hline 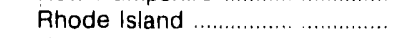 & 98 & 101 & 199 & 0 & 1,961 & 22,506 \\
\hline 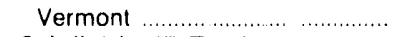 & 4,301 & 2,550 & 6,851 & 414 & 1,517 & - \\
\hline 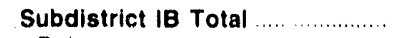 & 97,439 & 46,842 & 144,281 & 4,255 & 401,417 & $4,330,729$ \\
\hline Delaware & 4,854 & 687 & 5,541 & 8 & 14,312 & 173,374 \\
\hline 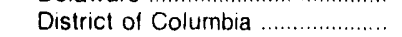 & - & - & - & - & 71,970 & - \\
\hline 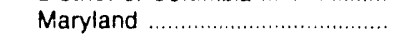 & 20,811 & 3,108 & 23,920 & 851 & 63,558 & 404,651 \\
\hline 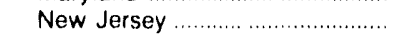 & 12,213 & 6,901 & 19,113 & 4 & 54,808 & 525,982 \\
\hline 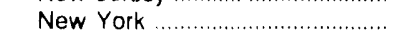 & 29,509 & 19,360 & 48,870 & 2,340 & 114,115 & $2,784,305$ \\
\hline Pennsylvania. & 30,052 & 16,786 & 46,838 & 1,052 & 82,654 & 442,417 \\
\hline 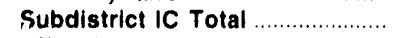 & 230,638 & 4,197 & 234,835 & 2,689 & 186,446 & $1,960,456$ \\
\hline 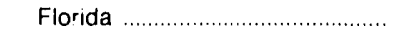 & 47,494 & 39 & 47,533 & 36 & 79,629 & $1,726,877$ \\
\hline 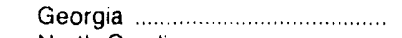 & 82,960 & 5 & 82,966 & 27 & 16,155 & 618 \\
\hline 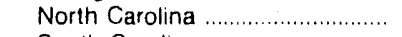 & 37,854 & 1,730 & 39,584 & 873 & 22,419 & - \\
\hline South Carolina $\ldots . . . \ldots \ldots \ldots \ldots \ldots \ldots \ldots \ldots \ldots \ldots \ldots$ & 26,966 & 428 & 27,394 & 605 & 11,880 & - \\
\hline 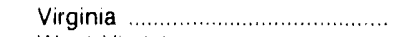 & 31,132 & 1,266 & 32,398 & 733 & 33,892 & 232,962 \\
\hline 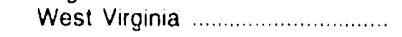 & 4,232 & 728 & 4,960 & 416 & 22,472 & - \\
\hline PAD District \| Total ........................ & $1,317,155$ & 76,265 & $1,393,420$ & 11,605 & 188,359 & 197,418 \\
\hline 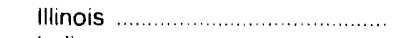 & 159,084 & 11,494 & 170,578 & 580 & 24,651 & 71,844 \\
\hline Indiana & 76,272 & 7,316 & 84,188 & 899 & 29,115 & - \\
\hline lowa & 139,896 & 7,688 & 147,584 & 598 & 4,832 & - \\
\hline 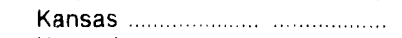 & 129,682 & 2,722 & 132,404 & 531 & 7,468 & - \\
\hline Kentucky & 36,702 & 1,900 & 38,602 & 942 & 17,107 & - \\
\hline 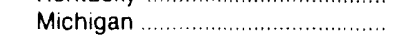 & 56,980 & 2,850 & 59,830 & 320 & 37,769 & 87,400 \\
\hline 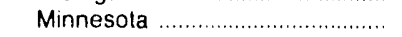 & 124,001 & 5,423 & 129,423 & 2,162 & 6,384 & 7 \\
\hline 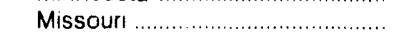 & 77,957 & 1,366 & 79,324 & 412 & 9,620 & 14,460 \\
\hline 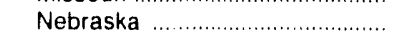 & 143,507 & 2,130 & 145,637 & 451 & 3,902 & 2,531 \\
\hline 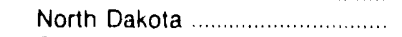 & 84,686 & E.205 & 89,892 & 16 & 4,302 & 243 \\
\hline Ohio & 67,887 & 6.931 & 74,818 & 3,501 & 21,269 & 20,933 \\
\hline 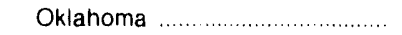 & 41,123 & - & 41,123 & 101 & 542 & - \\
\hline 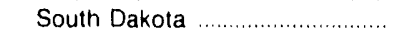 & 66,814 & 7,683 & 74,497 & 214 & 862 & - \\
\hline 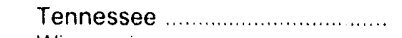 & 39,575 & 22 & 39,597 & 438 & 3,447 & - \\
\hline 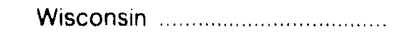 & 72,988 & 12,935 & 85,923 & 441 & 17,090 & - \\
\hline PAD District III Total. & 728,130 & 11,887 & 740,018 & 15,844 & 114,885 & 75,420 \\
\hline Alabama & 59,101 & 1,220 & 60,320 & 272 & 10,486 & - \\
\hline 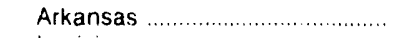 & 91,831 & 275 & 92,106 & 1,383 & 11,073 & 5,726 \\
\hline 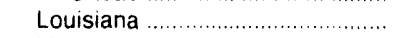 & 91,536 & 218 & 91,754 & 3,589 & 20,371 & 8,524 \\
\hline 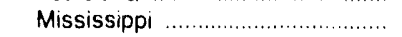 & 133,228 & 10 & 133,238 & 4,480 & 5.072 & 51,407 \\
\hline 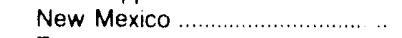 & 16,925 & - & 16,925 & 424 & 6,351 & 1,775 \\
\hline 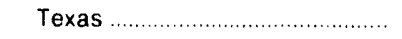 & 335,510 & 10,165 & 345,675 & 5,695 & 61,532 & 7,988 \\
\hline PAD District IV Total ...................... & 193,012 & 3,138 & 196,150 & 377 & 18,933 & 39 \\
\hline Colorado & 53,986 & 671 & 54,657 & 301 & 5,161 & 3 \\
\hline ldaho & 67,921 & 345 & 68,266 & 11 & 27 & - \\
\hline Montana & 52.549 & 1,310 & 53,859 & 44 & 2,552 & - \\
\hline 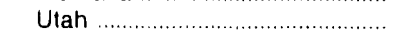 & 9,343 & 313 & 9,656 & 11 & 4,293 & - \\
\hline 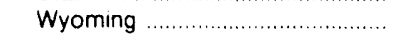 & 9,214 & 499 & 9,713 & 11 & 6,899 & 36 \\
\hline PAD District $V$ Total & 466,616 & 2,627 & 469,243 & 1,089 & 129,313 & 923,621 \\
\hline 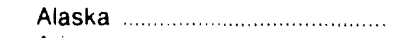 & 257 & - & 257 & - & 21,953 & - \\
\hline Arizona & 31,647 & - & 31,647 & 27 & 4,382 & 3,393 \\
\hline 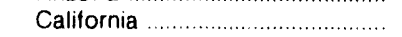 & 302,115 & 10 & 302,124 & 823 & 19,719 & 372,937 \\
\hline 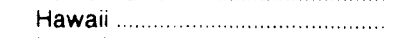 & 10,013 & 1,119 & 11,132 & 0 & 74,240 & 522,447 \\
\hline 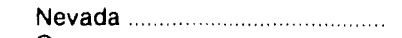 & 5,048 & 139 & 5,187 & 70 & 2,639 & 24,844 \\
\hline Oregon & 49,175 & 70 & 49,245 & 88 & 5,363 & 20,0 \\
\hline 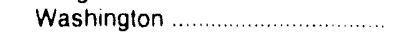 & 68,362 & 1,288 & 69,650 & 81 & 1,017 & - \\
\hline
\end{tabular}

Cashes $(\cdot)$.. No data reported.

Note: Totals may not equal sum of components due to independent rounding

Sources: - Energy Information Administration Form ElA-821, "Annual Fuel Oil and Kerosene Sales Feport," for 1989.

- Electric Utility data are based on data obtained on Form EIA.759, "Monthly Power Plant Report," and Form FERC.423, "Monthly Report of Cost and Quality of Fuels for Electric Plarts."

- Energy Information Administration's "Petroleum Supply Annual," Volume 1, 1989, (DOE/EIA-0340(89)/1). 

Table 23. Adjusted Sales for Transportation Use: Distillate Fuel Oil and Residual
Fuel Oil, 1989

(Thousand Gallons)

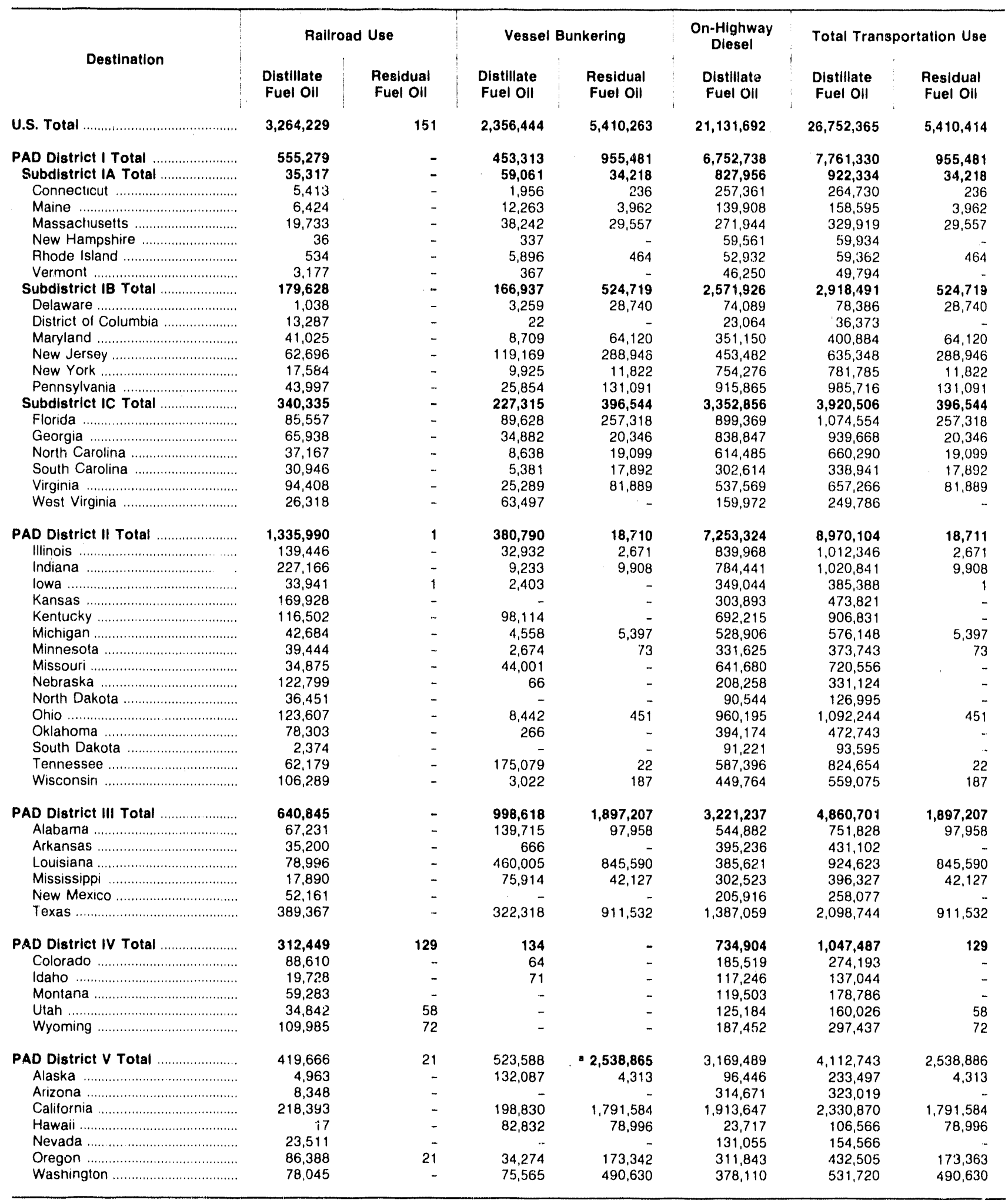

- See Technical Note 3 for adjustment to vessel bunkering data

Dashes $(-)$ :- No data reported.

Note: Totals may not equal sum of components due to independent rounding.

Sources: - Energy Information Administration Form EIA-821, "Annual Fuel Oil and Kerosene Sales Report," for 1989.

- Raiiroad data for distillate fuel oil are based on Association of American Railroads' statistics for railroads of Class I and Amtrak Companies.

- Vessel Bunkering data for residual fuel oil are based on EIA estimates of intercoastal bunkering and the Bureau of the Census Report, "United States Foreign Trade, Bunker Fuels."

- On-Highway Diesel data are Federal Highway Administation statistics of highway special fuels use.

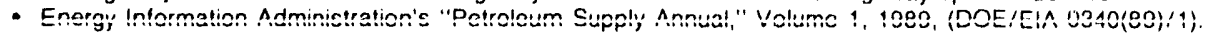


Table 24. Adjusted Sales for Military, Off-Highway, and All Other Uses: Distillate Fuel Oil, Residual Fuel Oil, and Kerosene, 1989

(Thousand Gallons)

\begin{tabular}{|c|c|c|c|c|c|c|c|c|c|c|}
\hline \multirow{2}{*}{ Destination } & \multicolumn{3}{|c|}{ Military } & \multicolumn{4}{|c|}{$\begin{array}{l}\text { Off-Highway Diesel } \\
\text { Distillate Fuel Oil }\end{array}$} & \multicolumn{3}{|c|}{ All Other } \\
\hline & Diesel & $\begin{array}{c}\text { Other } \\
\text { Distilla- } \\
\text { te }\end{array}$ & $\begin{array}{c}\text { Total } \\
\text { Distillate }\end{array}$ & $\begin{array}{l}\text { Residual } \\
\text { Fuel Oil }\end{array}$ & Construction & Other & Total & $\begin{array}{l}\text { Distillate } \\
\text { Fuel Oil }\end{array}$ & $\begin{array}{l}\text { Residual } \\
\text { Fuel Oll }\end{array}$ & Kerosene \\
\hline U.S. Total & 859,341 & 68,975 & 928,316 & 105,255 & $1,454,992$ & 182,505 & $1,637,497$ & 29,106 & 36,298 & 115,750 \\
\hline PAD District I Total & 85,164 & 56,203 & 141,367 & 90,853 & 424,924 & 36,712 & 461,637 & 11,307 & 84 & 24,205 \\
\hline Subdistrict IA Total & 10,919 & 4,183 & 15,102 & 25,823 & 60,350 & 6.152 & 66,502 & 597 & - & 3,908 \\
\hline Connecticut ................ & 64 & 547 & 611 & 3,097 & 18,681 & 32 & 18,713 & 480 & - & 2,898 \\
\hline Maine & 2,723 & 261 & 2,984 & 4,085 & 9,045 & 3,790 & 12,836 & 39 & - & 259 \\
\hline Massachuselts …................. & 1,267 & 721 & 1,988 & 14,388 & 18,204 & 15 & 18,219 & - & - & 375 \\
\hline New Hampshire .................. & 304 & 1,237 & 1,542 & 1,049 & 6,436 & 1,237 & 7,674 & - & - & 223 \\
\hline Rhode Island & 6,490 & 1,258 & 7,748 & 2,850 & 3,342 & 1 & 3,344 & 79 & - & 18 \\
\hline Vermont & 71 & 159 & 229 & 354 & 4,640 & 1,077 & 5,717 & . & - & 35 \\
\hline Subdistrict IB Total. & 27,369 & 28,260 & 55,629 & 40,918 & 171,541 & 9,455 & 180,995 & 3,338 & - & 17,856 \\
\hline Delaware & & 85 & 85 & & 4,199 & 3 & 4,202 & - & - & 17 \\
\hline District of Columbia ............ & 7 & 2,989 & 2,996 & 2,115 & 31 & - & 31 & - & - & - \\
\hline Maryland & 14,786 & 7,550 & 22,336 & 23,688 & 31,034 & 1,131 & 32,165 & 2,569 & - & 92 \\
\hline New Jersey & 4,125 & 4,999 & 9,124 & 2,892 & 50,622 & 1,830 & 52,452 & 48 & - & 1,751 \\
\hline New York .... & 5,933 & 4,477 & 10,410 & 8,815 & 34,610 & 5,689 & 40,298 & 3 & - & 15,137 \\
\hline 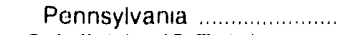 & 2,518 & 8,159 & 10,677 & 3,408 & 51,045 & 802 & 51,847 & 718 & - & 859 \\
\hline Subdistrict IC Total & 46,876 & 23,760 & 70,636 & 24,112 & 193,034 & 21,105 & 214,139 & 7,371 & 84 & 2,441 \\
\hline Florida ……....................... & 20,292 & 13 & 20,305 & 7,460 & 62,124 & 3,503 & 65,627 & 1,307 & - & 411 \\
\hline Georgia & 8,682 & 471 & 9,153 & 1,947 & 35,307 & 2,872 & 38,179 & 1,610 & - & 200 \\
\hline North Carolina ......................... & 6,797 & 997 & 7,794 & 4,214 & 25,257 & 1,398 & 26,655 & 2,234 & 34 & 439 \\
\hline South Carolina ....................... & 2,078 & 478 & 2,557 & 6,343 & 15,763 & 10,839 & 26,601 & 908 & - & 697 \\
\hline 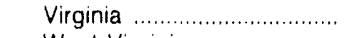 & 8,914 & 21,750 & 30,664 & 4,149 & $35,5,38$ & 1,182 & 36,720 & 1,299 & - & 526 \\
\hline West Virginia & 111 & 51 & 163 & - & 19,046 & 1,312 & 20,358 & 13 & - & 168 \\
\hline PAD District || Total .............. & 38,954 & 5,617 & 44,570 & 4,291 & 321,326 & 19,024 & 340,350 & 13,925 & 549 & 6,322 \\
\hline 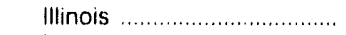 & 3,873 & 557 & 4,430 & - & 37,156 & 396 & 37,553 & 161 & - & 1,082 \\
\hline Indiana & 6,441 & 128 & 6,569 & - & 28,023 & 1,442 & 29,465 & 2,819 & - & 183 \\
\hline lowa & 246 & 26 & 272 & 10 & 8,602 & 994 & 9,595 & 1,876 & - & 338 \\
\hline 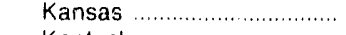 & 751 & 104 & 855 & - & 10,865 & 1,487 & 12,352 & 1,601 & - & 134 \\
\hline 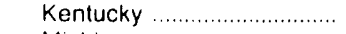 & 4,550 & 869 & 5,419 & - & 21,153 & 658 & 21,811 & 19 & - & 420 \\
\hline Michigan ............................. & 2,636 & 510 & 3,146 & - & 43,959 & 1,438 & 45,397 & 1,744 & 74 & 411 \\
\hline Minnesota ............................... & 1,818 & 212 & 2,030 & - & 23,632 & 2,532 & 26,163 & 1,818 & 153 & 63 \\
\hline Missouri ….............................. & 3,204 & 1,790 & 4,994 & 3,709 & 20,477 & $1,02: 2$ & 21,499 & 1,165 & - & 803 \\
\hline Nebraska & 988 & 75 & 1,063 & - & 16,834 & 248 & 17,083 & - & 19 & 12 \\
\hline North Dakota ........................... & 1,334 & 10 & 1,344 & - & 3,942 & 567 & 4,509 & 194 & 303 & 12 \\
\hline Ohio & 8,432 & 165 & 8,597 & - & 27,145 & 819 & 27,963 & 335 & - & 2,328 \\
\hline$\ldots+\ldots \ldots \ldots \ldots \ldots$ & 1,099 & 392 & 1,491 & - & 20,653 & 1,394 & 22,047 & 227 & - & 65 \\
\hline South Dakota ....................... & 486 & 26 & 512 & 2 & 5,543 & 100 & 5,642 & 540 & - & 35 \\
\hline Tennessee ............................. & 2,301 & 484 & 2,785 & 571 & 24,604 & 2,015 & 26,619 & 650 & - & 357 \\
\hline Wisconsin & 795 & 269 & 1,064 & - & 28,739 & 3,913 & 32,652 & 776 & - & 79 \\
\hline rict III Total & 442,078 & 101 & 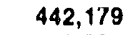 & 1,228 & 33 & 39 & 37 & 3,425 & - & 84,048 \\
\hline …………… & 6,235 & 100 & 6,335 & 1,229 & 53,194 & $10,67 ?$ & 63,870 & 1,999 & - & 4,201 \\
\hline 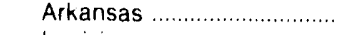 & 2,069 & - & 2,069 & - & 22,855 & 9,274 & 32,128 & 63 & ... & 672 \\
\hline$\ldots+\ldots \ldots \ldots \ldots \ldots$ & 83,075 & - & 83,075 & - & 53,626 & 1,348 & 54,974 & 275 & - & 19,230 \\
\hline Mississippl …………….......... & 73,437 & 1 & 73,439 & - & 27,743 & 5,833 & 33,576 & 162 & - & 6,870 \\
\hline New Mexico .......................... & 912 & - & 912 & - & 20,578 & 906 & 21,484 & 48 & - & 653 \\
\hline 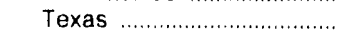 & 276,348 & - & 276,348 & - & 159,960 & 10,976 & 170,936 & 879 & - & 52,423 \\
\hline PAD District IV Total & 11,504 & 2,244 & 13,748 & - & 82,778 & 10,833 & 93,611 & 450 & 1,524 & 174 \\
\hline Colorado & 8,353 & - & 8,353 & - & 26,900 & 455 & 27,355 & 46 & - & 159 \\
\hline Idaho ......... & 738 & - & 738 & - & 11,009 & 2,864 & 13,872 & 26 & - & 2 \\
\hline 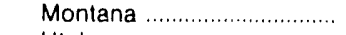 & 218 & 132 & 351 & - & 8,981 & 7,008 & 15,989 & 25 & 1,331 & 2 \\
\hline Utah & 2,008 & 2,111 & 4,120 & - & 18,214 & 337 & 18,551 & - & - & 5 \\
\hline 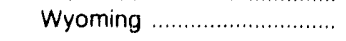 & 186 & - & 186 & - & 17,674 & 170 & 17,844 & 353 & 193 & 6 \\
\hline PAD District $V$ Total & 281,642 & 4,810 & 286,451 & 8,882 & 288,007 & 76,921 & & - & 34,141 & 1,001 \\
\hline Alaska & 33,958 & 117 & 34,075 & - & 8,588 & 5,393 & 13,980 & - & - & 20 \\
\hline Arizona & 2,246 & 7 & 2,253 & - & 35,516 & 2,461 & 37,978 & - & - & 104 \\
\hline$\ldots \ldots \ldots \ldots \ldots \ldots \ldots$ & 205,471 & - & 205,471 & - & 167,842 & 25,418 & 193,260 & - & - & 644 \\
\hline Hawaii & 31,149 & - & 31,149 & 3,647 & 5,371 & 56 & 5,428 & - & 34,069 & - \\
\hline 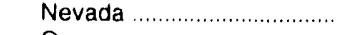 & 3,613 & 787 & 4,401 & - & 23,805 & 261 & 24,066 & - & - & 191 \\
\hline 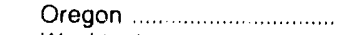 & 682 & 51 & 733 & - & 15,795 & 28,782 & 44,577 & - & 72 & 27 \\
\hline Washington ........................... & 4,523 & 3,847 & 8,370 & 5,235 & 31,090 & 14,550 & 45,640 & - & $=$ & 15 \\
\hline
\end{tabular}

Dashes $(\cdot)=$ No data reported.

Note: Totals may not equal sum of components due to independent rounding.

Sources: - Energy Information Administration Form ElA-821, "Annual Fuel Oil and Kerosene Sales Report," for 1989

- Military data for residual fuel oll are based on Defense Fuel Supply Center's "DEIS Report."

- Energy information Administration's "Petroleum Supply Annual," Volume 1, 1989, (DOE/EIA-0340(89)/1). 


\section{Technical Notes}

\section{Note 1: ElA-821: Annual Fuel Oil and Kerosene Sales Report, 1989}

\section{Background}

The EIA-821, "Annual Fuel Oil and Kerosene Sales Report" survey was implemented to meet Energy Information Administration (EIA) legislative mandates and data user needs. Form EIA-821 is used to gather data on the sales to end users of distillate fuel oil, residual fuel oil, and kerosene. The data are used to determine the level of demand by end-use category and product at the State, regional, and national levels. This mandatory report is authorized by the Federal Energy Administration Act of 1974 (P. L. 93-275).

The predecessor survey to the ElA-821 was the EIA-172, "Sales Report of Fuel Oil and Kerosene" survey. The EIA-172 was conducted for reference years 1979 through 1982. Due to a serious deterioration in the sample of respondents during the 4-year period, the Form ElA-172 data were not collected for 1983. Instead, estimates for 1983 were published and a new sample of respondents was designed and selected from an improved sampling frame. The EIA-821 survey commenced with reference year 1984 .

\section{Discussion of Sampling Frame}

The target population for the fuel oil and kerosene sales survey is the universe of companies that sell fuel oil and kerosene to end users.

The EIA-863 data base provided the sampling frame for the 1989 ElA-821 survey. The EIA-863, "Petroleum Product Sales Identification Survey," was mailed 10 approximately 27,000 companies in January 1989 to collect 1988 State-level sales volume data for No. 2 distillate, residual fuel, and motor gasoline. Companies also indicated if they sold kerosene. The No. 2 distillate data were further identified by residential and nonresidential end use, and non-end-use sales; the residual and motor gasoline data were identified by end use and non-end-use sales. The mailing list for the EIA-863 survey was constructed by merging and unduplicating the 1985 frame file and more than 51 State and commercial lists. In addition, company/State-level volumes for distillate fuel oil, residual fuel oil, and motor gasoline from the 1988 EIA-821 survey were merged with the EIA-863 to yield a combined data file. The transformed and edited version of the frame was then used to design and select the 1989 EIA-821 sample.

It should be noted that truck stops selling No. 2 diesel fuel were not specifically included in the ElA-863 survey. Therefore, the EIA-821 survey does not include all sales of No. 2 distillate through retail truck stops. However, to compensate for this shortage in coverage, data from the Federal Highway Administration are presented in the tables for on-highway diesel sales.

The sampling frame a!so lacked full coverage of kerosene retailers, many of whom are hardware stores and service stations. To account for all kerosene volumes supplied to the marketplace, aggregate reported kerosene volumes are set equal to the products supplied volumes published in the Petroleum Supply Annual.

\section{Discussion of the Sample Design}

The purpose of the EIA-821 sample design was to produce State-level estimates of total sales for the following five target variables: (1) residential No. 2 distillate, (2) other retail No. 2 distillate, (3) wholesale No. 2 distillate, (4) retail residual, and (5) wholesale residual. A separate sample was initially designed for each target variable.

Companies were divided into two basic types of strata: certainty and noncertainty. Companies selected with certainty were:

- all refiners and gas plant operators

- companies doing business in four or more States

- companies accounting for 5 percent or more of the distillate or residual fuel oil volume for any target variable or particular end-use category sold in a State 
- companies accounting for 5 percent or more of the kerosene volume sold in a State in earlier EIA-821 surveys

- augmented frame units, e.g., vessel bunkering for which no attribute data were available.

Firms determined not to be selling distillate or residual fuel oil or kerosene were removed from the frame file. Volumes for nonrespondents to the EIA-863 who had reported in the previous frame survey or in a sample survey were imputed. The imputed nonrespondents and the noncertainty companies were stratified by sales volume for each target variable. Strata boundries were determined using the Dalenius-Hodges procedures, allowing the number of strata and the strata boundries to vary by State. Nonrespondents for whom no previous information was available were classified in a separate stratum and sampled with reduced probabilities of selection.

Neyman allocation was used to allocate the number of companies required for each strata to obtain the required leveis of accuracy: a 2.5 percent coefficient of variation for residual fuel oil and a 5 percent coefficient of variation for distillate fuel oil. Sample selections were then performed simultaneously from the same random list for the five samples to produce a final linked sample of approximately 5,500 companies. This method reduced the total survey sample size while improving volume estimates. Selected companies were asked to report sales by end-use category of distillate and residual fuel oil and kerosene.

\section{Imputation and Estimation}

Survey data gathered from the respondents may contain incomplete reporting, total nonresponse, or values that fail editing. Missing data are estimated, or implicitly imputed for, using the strata means and treated the same as reported data. The weighted estimate is defined as follows:

$$
\hat{V}=\Sigma_{h} N_{h}\left(\Sigma_{i} W_{h i} V_{h i} / \Sigma_{i} W_{h i}\right)
$$

where:

$$
\begin{aligned}
& V=\text { total estimated volume, } \\
& \Sigma_{h}=\text { denotes summation over strata, }
\end{aligned}
$$

$$
\begin{aligned}
N_{h}= & \text { stratum population for stratum } \mathrm{h}, \\
\Sigma_{i}= & \text { denotes summation over units within } \\
& \text { stratum } \mathrm{h}, \\
V_{h i}= & \text { volume reported for unit } \mathrm{i} \text { in stratum } \mathrm{h}, \\
W_{h i}= & \text { weight attached to unit } \mathrm{i} \text { in stratum } \mathrm{h} .
\end{aligned}
$$

where: $W_{h i}$ is inversely proportional to the probability of inclusion in the linked sample.

The degree of imputation by product and end use at the U.S. total level ranged as follows for the 1989 EIA-821 data: distillate, 0.4 percent for all other use to 6.8 percent for residential use; residual fuel oil, 0.006 percent for all other use to 8.8 percent for commercial use; and kerosene, 1.0 percent for industrial use to 4.7 percent for all other use.

\section{Collection Methods}

The EIA-821 form was mailed in January 1990 to all companies selected for participation in the 1989 reference year survey. The completed form was due to EIA on March 1, 1990. A second request was sent by certified mail to nonrespondents approximately 1 month after the filing deadline. Follow-up telephone calls were made to collect outstanding data and to verify reported data. Late submissions and resubmissions were processed wher received.

\section{Data Processing}

\begin{abstract}
As EIA-821 forms were received, they were logged into an automated Survey Control File which maintained company identification and survey form status information for each company selected to participate in the survey. The data were reviewed manually, en tered onto the computer files, and then processed through an automated edit program which detected missing data and outlying values. Data that failed the edits were resolved through telephone calls to the data reporters and corrections were entered onto the computer files. Preliminary estimates were generated and processed through a series of validation procedures to flag and rectify potential misreporting of data. Statistical reports, including publication tables, were produced using only acceptable and verified data.
\end{abstract}




\section{Note 2: Reliability of the Data}

Two types of errors are associated with data produced from a sample survey---sampling errors and nonsampling errors. Sampling errors oecur because the estimates are based on a sample rather than on a census. The particular sample used for the EIA-821 survey is one of a large number of samples of equal size which could have been selected from the sumpling frame using the same sample design. Each of these samples would produce a different estimate. If the estimates were averaged over all possible sumples, the result would be the same as the estimate derived from a census of the sampling frame. The sampling error is a measure of variability among the estimates from all possible samples and, thus, is a measure of the precision with which an estimate from a particular sample approximates the results of a census.

Nonsampling errors and possibly biases can arise from a number of sources: (1) inability to obtain information about all cases in the sample, (2) response errors, (3) differences in the interpretation of questions or definitions, (4) mistakes in recording or coding of the data obtained, and (5) other errors of collection, response, coverage, and estimation for missing data. Bias is the difference between the average of the estimates over all possible samples of the same size and design, and the true value being estimated. It is not possible to estimate bias using the results of one sample.

Data obtained from alternate sources are not subject to sampling errors, but may be subject to nonsampling errors, the magnitudes of which are unknown. For example, kerosene estimates may have more nonsampling errors due to a lack of coverage of kerosene retailers. Nonsampling errors for survey estimates and estimates adjusted to alternate sources cannot be determined, but attempts are made throughout survey processing to minimize this type of error.
Data in Tables 1 through 12 are based on survey data which are subject to sampling errors. Coefficients of variation, which are estimates of sampling errors, are presented for the retail target variables in the following table for the 1989 survey. The coefficients of variation (CV) were estimated by:

$C V(\hat{V})=\frac{\sqrt{V A R(\hat{V})}}{\hat{V}}$

where:

$$
\begin{aligned}
& \operatorname{VAR}(\hat{V})=\sum_{h} n_{h}\left(1-\frac{n_{h}}{N_{h}}\right) S_{h}^{2} \\
& S_{h}^{2}=\frac{\sum_{i=1}^{n_{h}} W_{i}^{2} V_{i}^{2}+\bar{V}_{h}^{2} \sum_{i=1}^{m_{h}} W_{i}^{2}-2 \bar{V}_{h} \sum_{i=1}^{m_{h}} W_{i}^{2} V_{i}}{n_{h}-1} \\
& \bar{V}_{h}=\frac{\sum_{i=1}^{m_{h}} W_{i} V_{i}}{\sum_{i=1}^{m_{h}} W_{i}} \\
& \hat{V}=\text { total estimated volume, } \\
& N_{h}=\text { stratum population for stratum } \mathrm{h}, \\
& n_{h}=\text { number of sample units in stratum } \mathrm{h}, \\
& V_{i}=\text { volume for unit } \mathrm{i}, \\
& W_{i}=\text { weight for unit } \mathrm{i} .
\end{aligned}
$$

Response rates also offer some indication of the reliability and comprehensiveness of survey results. For the 1989 EIA-821 survey, the overall response rate (the number of submissions received, divided by the number of submissions solicited and expected, times 100) was 92.3 percent. 
Coefficients of Variation for Sales of Fuel Oil, 1989

\begin{tabular}{|c|c|c|c|}
\hline Geographic Area & $\begin{array}{c}\text { Residential Distillate } \\
\text { Fuel Oil }\end{array}$ & $\begin{array}{c}\text { Non-Residential } \\
\text { Retall Distillate } \\
\text { Fuel Oll }\end{array}$ & $\begin{array}{c}\text { Retall } \\
\text { Residual Fuel OII }\end{array}$ \\
\hline U.S. Average & 1.8 & 0.8 & 0.3 \\
\hline PAD Distrlct I Average & 2.2 & 1.3 & .3 \\
\hline Subdistrict IA Average ..................... & 2.8 & 1.8 & .5 \\
\hline 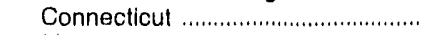 & 6.8 & 4.8 & .2 \\
\hline 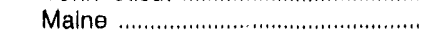 & 5.8 & 3.2 & .1 \\
\hline 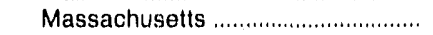 & 4.5 & 3.1 & 1.0 \\
\hline 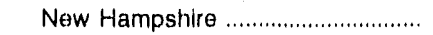 & 6.6 & 2.7 & .0 \\
\hline Rhode Island ................................... & 4.2 & 3.3 & .1 \\
\hline 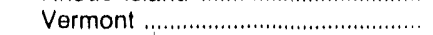 & 4.8 & 3.5 & .0 \\
\hline Subdistrict IB Average ....................... & 3.5 & 1.7 & .6 \\
\hline Delaware ................................... & 3.5 & 1.7 & .0 \\
\hline District of Columbia ............................. & 4.1 & .1 & .0 \\
\hline 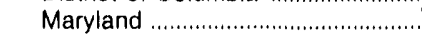 & 4.7 & 2.8 & .2 \\
\hline New Jersey ......................................... & 4.5 & 2.8 & .3 \\
\hline 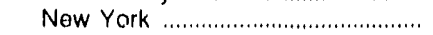 & 7.2 & 2.6 & 1.1 \\
\hline 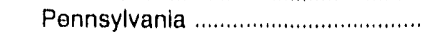 & 4.8 & 4.2 & .7 \\
\hline Subdistrict IC Average & 3.2 & 2.5 & .2 \\
\hline Florida & 13.7 & 6.2 & .1 \\
\hline 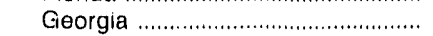 & 7.3 & 7.0 & 1.5 \\
\hline North Carolina & 5.4 & 3.0 & 2.1 \\
\hline South Carolina & 7.6 & 6.0 & .2 \\
\hline 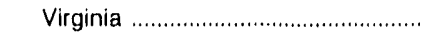 & 5.3 & 5.0 & 3 \\
\hline 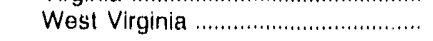 & 6.6 & 3.1 & .0 \\
\hline PAD Dlstrict $\|$ Average & 2.1 & .9 & .4 \\
\hline 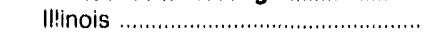 & 9.4 & 3.3 & 8 \\
\hline 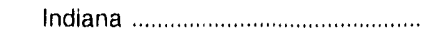 & 5.8 & 3.0 & .3 \\
\hline 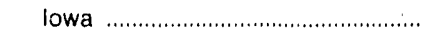 & 6.9 & 3.0 & 4.0 \\
\hline 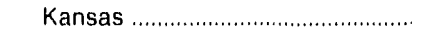 & 11.2 & 2.9 & .0 \\
\hline 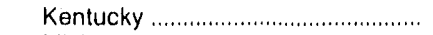 & 4.8 & 2.0 & .1 \\
\hline 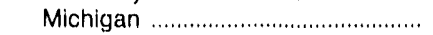 & 5.4 & 2.9 & 1.6 \\
\hline 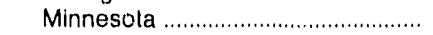 & 5.4 & 4.5 & .8 \\
\hline 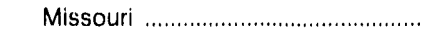 & 7.1 & 3.5 & 1.2 \\
\hline 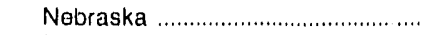 & 9.2 & 4.0 & .0 \\
\hline North Dakota ...................................... & 5.5 & 7.3 & .1 \\
\hline 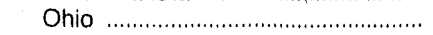 & 6.4 & 2.8 & .1 \\
\hline Oklahoma …..................................... & 62.5 & 5.8 & 0 \\
\hline 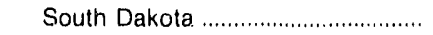 & 7.1 & 3.9 & .1 \\
\hline Tennessee & 8.8 & 2.4 & .0 \\
\hline 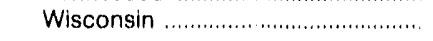 & 5.2 & 3.3 & 3.3 \\
\hline PAD District III Average .................... & 12.0 & 2.6 & .3 \\
\hline 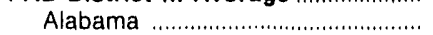 & 17.6 & 4.1 & .0 \\
\hline 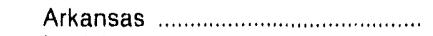 & 9.1 & 5.0 & .0 \\
\hline Louisiana …........................................ & 25.9 & 2.9 & .0 \\
\hline 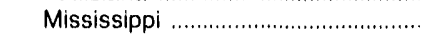 & 16.4 & 6.5 & .0 \\
\hline 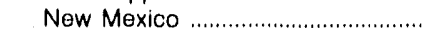 & 22.7 & 3.8 & .0 \\
\hline 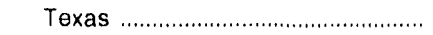 & 41.2 & 4.9 & .7 \\
\hline PAD District IV Average & 6.9 & 2.0 & .6 \\
\hline Colorado ....................................... & 11.5 & 3.6 & .0 \\
\hline 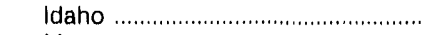 & 7.0 & 4.8 & .3 \\
\hline Montana & 19.2 & 8.0 & 1.7 \\
\hline 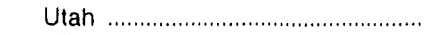 & 6.7 & 2.7 & .0 \\
\hline 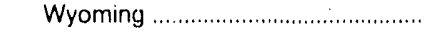 & 8.3 & 3.0 & .0 \\
\hline PAD District V Average & 2.4 & 2.1 & 1.1 \\
\hline 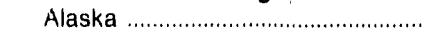 & 3.5 & 3.8 & .0 \\
\hline Arizona & .0 & 6.5 & .1 \\
\hline California & 10.5 & 3.4 & 1.8 \\
\hline 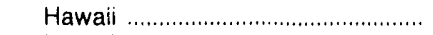 & .0 & 1.0 & .1 \\
\hline 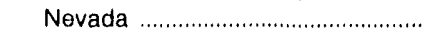 & 3.8 & 2.0 & .0 \\
\hline 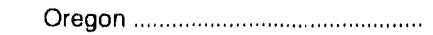 & 5.8 & 5.0 & 1.3 \\
\hline 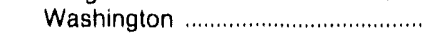 & 3.7 & 4.1 & .1 \\
\hline
\end{tabular}

Note: Coefficients of variation that are less than 0.1 and greater than zero . e displayed as 0.1

Source: - Energy Information Administration Form EIA-821, "Annual Fuel Oil and Kerosene Sales Report," for 1989 


\section{Note 3: Data Adjustments}

\section{Alternate Source Data}

After all preliminary tabulations were verified, comparisons were made between the survey results and available alternate source data. The following end-use categories were replaced by alternate source data at the U.S., Petroleum Administration for Defense (PAD) district, or State level:

\section{Tables 1 through 12:}

On-Highway Diesel. Distillate fuel oil by State was calculated from the Federal Highway Administration data on highway use of special fuels. Of the 1985 through 1989 special fuels, more than 98 percent is diesel.

\section{Tables 13 through 24:}

Electric Utility. National-level distillate and residual fuel oil sales for electric utility use were calculated from annual aggregations of data collected on forms EIA-759, "Monthly Power Plant Report," and FERC-423, "Monthly Report of Cost and Quality of Fuels for Electric Plants." Form EIA-759 consumption data for light oils include No. 2 fuel oil, kerosene, and jet fuel; the heavy oils include No. 4 fuel oil, residual fuel oil, and crude oil. Using receipt data from Form FERC-423, the No. 4 fuel oil receipts were deleted from the heavy oil consumption data and added to the light oil consumption data. The resultant distillate (light oils) and residual (heavy oils) consumption data were added to the stock change of light oils and heavy oils, respectively, from Form EIA-759 to obtain the estimate of sales. Allocations at the State level were based on the EIA-821 survey.

Military. The national data on residual fuel oil issued to defense installations (Defense Fuel Supply Center's Defense Energy Information System (DFSC DEIS) Report) were allocated by State based on the EIA-821 survey.

On-Highway Diese!. Distillate fuel oil by State was calculated from the Federal Highway Administration data on highway use of special fuels. Of the 1985 through 1989 special fuels, more than 98 percent is diesel.

Railroad. Distillate fuel oil used by Class I railroads and Amtrak at the U.S. level was obtained from the Association of American Railroads. State-level allocations were based on the EIA-821 survey.
Vessel Bunkering. Residual fuel oil used for foreign trade vessel bunkering at the PAD district level was obtained from the Bureau of Census, United States Foreign Trade, Bunker Fuels. To account for all vessel bunkering, including coastal trade, historically established PAD district-level ratios of total vessel bunkering use to foreign trade use were used to inflate the foreign trade bunkering values. The adjustment factor for PAD District V, however, was changed in 1988 and 1989. Had the PAD District $V$ estimate been adjusted using the historical factor, the vessel bunkering data in this PAD district and at the national level would have been 406,883 thousand gallons higher than the published estimates for 1988 and 413,834 thousand gallons higher than the published estimates for 1989.

Allocations at the State level were based on the EIA-821 survey, except for the States in PAD District II. For this region, which includes the Great Lakes, the EIA-821 survey results were used since the Census report, based on the U.S. Custom Service's data, did not provide adequate coverage.

\section{5 through 1989 Adjustments}

Kerosene volumes in Tables 1 through 12 were adjusted at the national level to equal products supplied volumes as published in the "Detailed Statistics" section of the Petroleum Supply Annual, 1985 through 1989. The 1989 survey resulted in 931,016 thousand gallons of kerosene at the national level. The products supplied volume at the national level for kerosene was $1,291,668$ thousand gallons. Hence, the adjustment factor at the national level for 1989 was 1.387 .

Volumes in Tables 13 through 24 were adjusted at the PAD district level to equal products supplied volumes. The adjustment factors for distillate and residual fuel and kerosene at the PAD district level for 1989 are as follows:

\section{Distillate Fuel Oil \\ - PAD District I: .87 \\ - PAD District II: .83 \\ - PAD District III: 1.37 \\ - PAD District IV: 1.03 \\ - PAD District V: .96}

\section{Residual Fuel Oil \\ - PAD District I: .41 \\ - PAD District II: 1.18 \\ - PAD District III: 12.37 \\ - PAD District IV: 6.77 \\ - PAD District V: 1.10}

\section{Kerosene \\ - PAD District I: .68}


- PAD District II: 1.50

- PAD District III: 14.16

- PAD District IV: 67

- PAD District V: 2.28

For Tables 13 through 24, total sales at the PAD district level equal the products supplied volumes as reported in the Petroleum Supply Annual (PSA), 1985 through 1989. For example, the 1989 survey yielded a volume for distillate residential use in PAD District il of 1,404,069 thousand gallons. The total distillate volume for PAD District II was 5,583,539 from the survey, after subtracting the volume coming from alternate sources. The product supplied total distillate volume for PAD District II was 4,634,271 after removing alternate source volume. Hence, the adjusted 1989 estimate of distillate for residential use in PADD II was $(4,634,271 / 5,583,539) \times 1,404,069=1,165,361$.

For distillate fuel oil in Tables 13 through 24, the PAD district adjustments were made to each State and product-use category except on-highway diese! use, electric utility use, and railroad use. For residual fuel oil, the adjustments were made to each State and enduse category except military use, vessel bunkering use, and electric utility use.

\section{Note 4: End-Use Descriptions and Petroleum Product Definitions}

\section{End-Use Descriptions}

Residential Use. Includes sales for use in private households for space heating, water heating, cooking, etc. Sales to farm houses are reported under "Farm Use."

Commercial Use. Includes sales for use in nonmanufacturing establishments such as motels, apartment buildings, restaurants, retail stores, institutions, government buildings, schools, and laundries for space heating, water heating, and cooking. Volumes used by companies in the marketing and distribution of petroleum products are also included.

Industrial Use. Includes sales to mines, smelters, and plants engaged in producing manufactured products, processing and assembling goods, etc. Also includes space heating. Excludes oil company use, as defined below.

Oil Company Use. Includes sales to drilling companies, pipelines or other related oil companies not engaged in the selling of petroleum products. Includes fuel oil that was purchased or produced and used by company facilities for the operation of drilling equipment, other field or refinery operations, and space heating at petroleum refineries, pipeline companies, and oil-drilling companies. Oil used to bunker vessels is counted under vessel bunkering. Sales to other oil companies for field use are included, but sales for use as refinery charging stocks are excluded.

Farm Use. Includes sales for use in tractors, irrigation pumps and other agricultural machinery, as well as that used for crop drying, smudge pot fuel, space heating of buildings (including farm houses), cooking, and any other use by the agriculture industry.

Electric Utility Companies. Includes sales to electric utility companies, including that used for the generation of electricity. Volumes imported and used directly by electric utility companies are also included.

Railroad Use. Includes sales to railroads, for any use, including that used for heating buildings operated by railroads.

Vessel Bunkering. Includes sales for the fueling of commercial or private boats, such as pleasure craft, fishing boats, tugboats, and ocean-going vessels, including vessels operated by oil companies. Excluded are volumes sold to the U.S. Armed Forces.

On-Highway Diesel Use. Includes sales for use in motor vehicles.

Military Use. Includes sales to the Armed Forces, including volumes sold to the Defense Fuel Supply Center (DFSC) fol use by all branches of the Department of Defense (DOD).

Off-Highway Diesel Use. Includes sales for use in:

1. Construction. Construction equipment including earthtnoving equipment, cranes, stationary generators, air compressors, etc.

2. Other. Sales for off-highway uses other than construction. Sales for logging are included in this category. Volumes for off-highway diesel us by the agriculture industry are reported under "Farm Use."

All Other Uses. Sales for all other uses not included in any other end-use category are reported here.

\section{Definitions of Petroleum Products and Other Related Terms}

ASTM. The acronym for the American Society for Testing and Materials. 
Distillate Fuel Oil. A general classification for one of the petroleum fractions produced in conventional distillation operations. It is used primarily for space heat. ing, on- and off-highway diesel engine fuel (including rallroad engine fuel and fuel for agricultural machinery), and electric power generation. Included are products known as No, 1, No, 2, and No, 4 fuel oils and No, 1, No, 2, and No, 4 diesel fuels.

1. No, 1 Distillate, A petroleum distillate which meets the specifications for No. 1 heating or fuel oil as defined in ASTM D 396 and/or the specifications for $\mathrm{No}$. 1 diesel fuel as defined in ASTM Specification D 975.

a. No. 1 Diesel Fuel. A volatile distillate fuel oil with a boiling range between 300 and 575 degrees Fahrenheit and used in high-speed diesel engines generally operated under wide variations in speed and load. Includes type C-B die. sel fuel used for city buses and similar operations. Properties are defined in ASTM Specificarion D 975 .

b. No, 1 Fuel Oil. A light distillate fuel oil intended for use in vaporizing pol-type burners. ASTM Specification D 396 specifies for this grade maximum distillation temperatures of 400 degrees Fahrenheit at the 10-percent point and 550 degrees Fahrenheit at the 90-percent point, and kinematic viscosities between 1.4 and 2.2 centistokes at 100 degrees Fahrenheit.

2. No. 2 Distillate. A petroleum distillate which meets the specifications for No. 2 heating oil or fuel oil as defined in ASTM D 396 and/or the specifications for No. 2 diesel fuel as defined in ASTM Specification D 975.

a. No. 2 Diesel Fuel. A gas oil type distillate of lower volatility with distillation temperatures at the 90-percent point between 540 and 640 degrees Fahrenheit for use in high speed diesel engines generally operated under uniform speed and load conditions. Includes Type R-R diesel fuel used for railroad locomotive engines, and Type T-T for diesel-engine trucks. Properties are defined in ASTM Specification D 975 .

b. No. 2 Fuel Oil. A distillate fuel oil for use in atomizing type burners for domestic heating or for moderate capacity commercialindustrial burner units. ASTM Specification D 396 specifies for this grade distillation temperatures at the 90 -percent point between 540 and 640 degrees Fahrenheit, and kinematic viscosities between 2.0 and 3.6 centistokes at 100 degrees Fahrenheit.

3. No. 4 Fuel. A fuel oil for commercial burner installations not equipped for preheating facilities. It is used extensively in industrial plants. This grade is a blend of distillate fuel oil and residual fuel oil stocks that conforms to ASTM Specification D 396 or Federal Specification VV. F-815C; its kinematic viscosity is between 5.8 and 26.4 centistokes at 100 degrees Fahrenheit. Also included is No, 4-D, a fuel oil for low-and medium-speed diesel engines that conforms to ASTM Specification D 975.

Kerosene. A petroleum distillate that boils at a temperature between 300 and 550 degrees Fahrenheit, that has a flash point higher than 100 degrees Fahrenheit by ASTM Method D 56, that has a gravity range from 40 to 46 degrees API, and that has a burning point in the range of 150 degrees to 175 degrees Fahrenheit. Included are the two classifications recognized by A.STM Specification D 3699: No. 1-K and No. 2-K, and ail grades of kerosene called range or stove oil which have properties similar to No. 1 fuel oil, but with a gravity of about 43 degrees API and a maximum end-point of 625 degrees Fahrenheit. Kerosene is used in space heaters, cook stoves, and water heaters and is suitable for use as an illuminant when burned in wick lamps.

NOTE: Respondents to the EIA-821 survey were instructed to report all volumes in accordance with what the product was sold as, regardless of the actual specifications of that product. For example, if a No. 2 distillate was sold as a heating oil or fuel oil, the volume would be reported in the category "No. 2 Fuel Oil" even if the product conformed to the higher specifications of a diesel fuel.

PAD District. Petroleum Administration for Defense Districts

\section{PAD District I:}

Subdistrict IA. Connecticut, Maine, Massachusetts, New Hampshire, Rhode Island, and Vermont

Subdistrict IB. Delaware, District of Columbia, Maryland, New Jersey, New York, and Pennsylvania

Subdistrict IC. Florida, Georgia, North Carolina, South Carolina, Virginia, and West Virginia

\section{PAD District II:}

Illinois, Indiana, Iowa, Kansas, Kentucky, Michigan, Minnesota, Missouri, Nebraska, North Dakota, South Dakota, Ohio, Oklahoma, Tennessee, and Wisconsin

\section{PAD District III:}

Alabama, Arkansas, Louisiana, Mississippi, New Mexico, and Texas

PAD District IV: 
Colorado, Idaho, Montana, Utah, and Wyoming

\section{PAD District V:}

Alaska, Arizona, California, Hawaii, Nevada, Oregon, and Washington

Residual Fuel Oils. The topped crude of refinery operations, which includes No. 5 and No. 6 fuel oils, as defined in ASTM Specification D 396 and Federal
Specification, VV-F-815C; Navy Special fuel oil as defined in Military Specification MIL-F-859E including Amendment 2 (NATO symbol F-77); and Bunker C fuel oil. Residual fuel oil is used for the production of electric power, space heating, vessel bunkering, and various industrial purposes.

United States. The 50 States and the District of Columbia. 

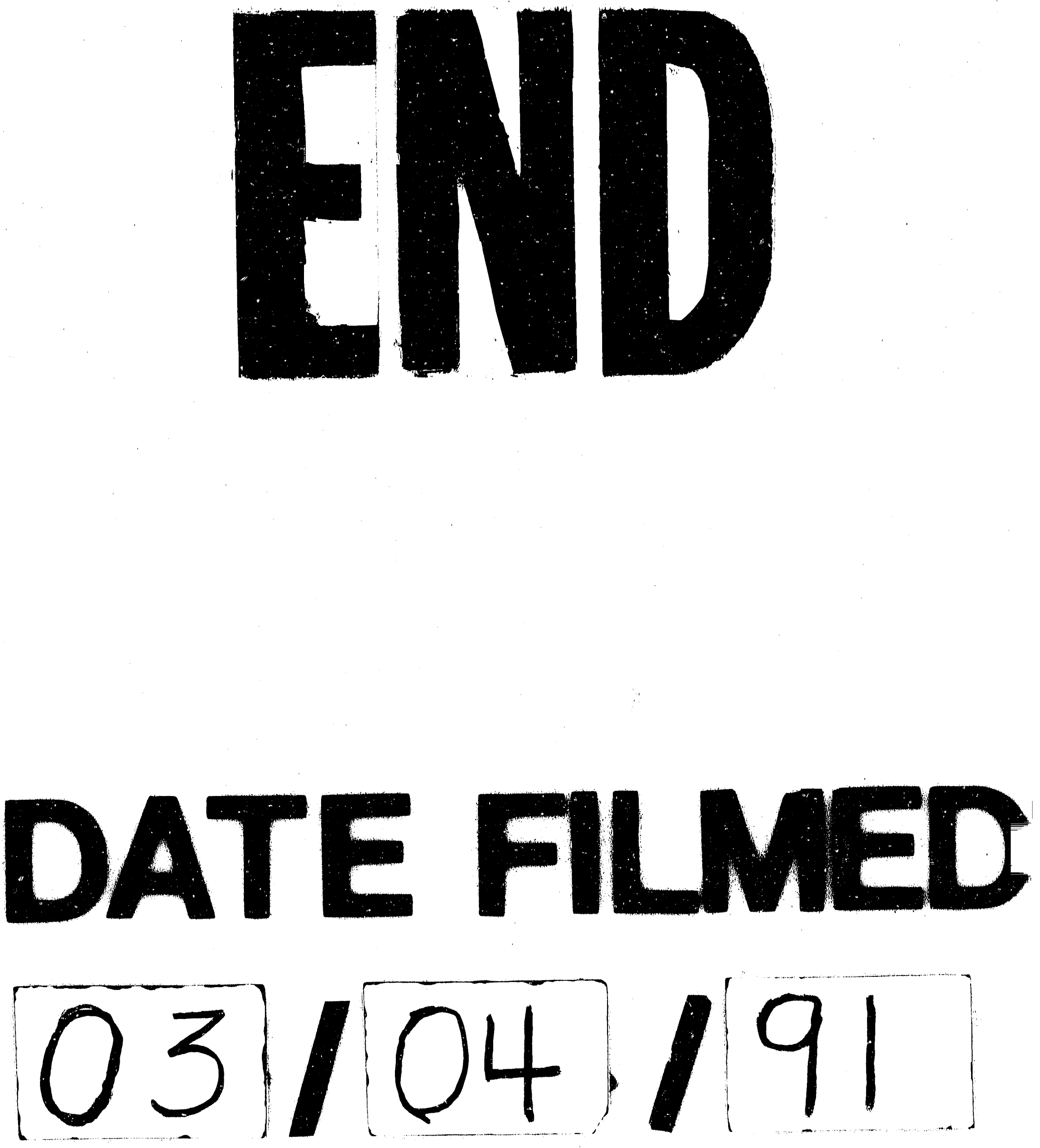
